\title{
REPRESENTATION THEORY OF QUANTIZED GIESEKER VARIETIES, I
}

\author{
IVAN LOSEV
}

\begin{abstract}
We study the representation theory of quantizations of Gieseker moduli spaces. We describe the categories of finite dimensional representations for all parameters and categories $\mathcal{O}$ for special values of parameters. We find the values of parameters, where the quantizations have finite homological dimension, and establish abelian localization theorem. We describe the two-sided ideals. Finally, we determine annihilators of the irreducible objects in categories $\mathcal{O}$ for some special choices of one-parameter subgroups.
\end{abstract}

\section{Contents}

1. Introduction

1.1. Gieseker moduli space and its quantizations

1.2. Classical cases

1.3. Main results

2. Preliminaries

2.1. Symplectic resolutions and their quantizations

2.2. Category $\mathcal{O}$

2.3. Standardly stratified structures and cross-walling functors

2.4. Wall-crossing functors and bijections

3. Gieseker varieties and their quantizations

3.1. Quantizations

3.2. Fixed point subvarieties and their quantizations

3.3. Symplectic leaves and slices

3.4. Harish-Chandra bimodules and restriction functors

4. Finite dimensional representations and structure of category $\mathcal{O}$

4.1. Finite dimensional representations

4.2. Proof of Theorem 1.4

5. Localization theorems

5.1. Results of McGerty and Nevins and consequences

5.2. Reduction to denominator $n$ and singular parameters

5.3. Number of simples in $\mathcal{O}\left(\mathcal{A}_{\lambda}(n, r)\right)$

5.4. Completion of proofs

6. Two-sided ideals and dimensions of supports

6.1. Two-sided ideals

6.2. Restriction functors for asymptotic chamber

6.3. Supports for asymptotic chambers

6.4. Cross-walling bijections

References 


\section{INTRODUCTION}

Our goal is to study the representation theory of quantizations of the Gieseker moduli spaces.

1.1. Gieseker moduli space and its quantizations. Let us explain constructions of the Gieseker moduli spaces and of their quantizations via Hamiltonian reduction.

Pick two vector spaces $V, W$ of dimensions $n, r$, respectively. Consider the space $R:=$ $\mathfrak{g l}(V) \oplus \operatorname{Hom}(V, W)$ and a natural action of $G:=\mathrm{GL}(V)$ on it. Then we can form the cotangent bundle $T^{*} R$, this is a symplectic vector space. Identifying $\mathfrak{g l}(V)^{*}$ with $\mathfrak{g l}(V)$ and $\operatorname{Hom}(V, W)^{*}$ with $\operatorname{Hom}(W, V)$ by means of the trace form, we identify $T^{*} R$ with $\mathfrak{g l}(V)^{\oplus 2} \oplus \operatorname{Hom}(V, W) \oplus \operatorname{Hom}(W, V)$. The action of $G$ on $T^{*} R$ is symplectic so we get the moment map $\mu: T^{*} R \rightarrow \mathfrak{g}$. It can be described in two equivalent ways. First, we have $\mu(A, B, i, j)=[A, B]-j i$. Second, the dual map $\mu^{*}: \mathfrak{g} \rightarrow \mathbb{C}\left[T^{*} R\right]$ sends $\xi \in \mathfrak{g}$ to the vector field $\xi_{R}$ (the infinitesimal action of $\xi$ ) that can be viewed as a polynomial function on $T^{*} R$.

Now pick a non-trivial character $\theta$ of $G$ and consider the open subset of $\theta$-stable points $\left(T^{*} R\right)^{\theta-s s} \subset T^{*} R$. For example, for $\theta=\operatorname{det}^{k}$ with $k>0$, the subset of semistable points consists of all quadruples $(A, B, i, j)$ such that ker $i$ does not contain nonzero $A$ and $B$-stable subspaces. Then we can form the GIT Hamiltonian reduction $\mathcal{M}^{\theta}(n, r):=$ $\mu^{-1}(0)^{\theta-s s} / G$, this is the Gieseker moduli space (for all choices of $\theta$ ). This a smooth symplectic quasi-projective variety of dimension $2 r n$ that is a resolution of singularities of the categorical Hamiltonian reduction $\mathcal{M}(n, r):=\mu^{-1}(0) / / G$. We note that the dilation action of $\mathbb{C}^{\times}$on $T^{*} R$ descends to both $\mathcal{M}^{\theta}(n, r), \mathcal{M}(n, r)$ (the corresponding action will be called contracting below). The resulting grading on $\mathbb{C}[\mathcal{M}(n, r)]$ is positive meaning that $\mathbb{C}[\mathcal{M}(n, r)]=\bigoplus_{i \geqslant 0} \mathbb{C}[\mathcal{M}(n, r)]_{i}$ (where $\mathbb{C}[\mathcal{M}(n, r)]_{i}$ is the $i$ th graded component) and $\mathbb{C}[\mathcal{M}(n, r)]_{0}=\mathbb{C}$.

Now let us explain how to construct quantizations of $\mathcal{M}(n, r)$ meaning filtered associative unital algebras $\mathcal{A}$ with gr $\mathcal{A} \stackrel{\sim}{\rightarrow} \mathbb{C}[\mathcal{M}(n, r)]$ (an isomorphism of graded Poisson algebras). Take $\lambda \in \mathbb{C}$ and set

$$
\mathcal{A}_{\lambda}(n, r):=(D(R) /[D(R)\{x-\lambda \operatorname{tr} x, x \in \mathfrak{g}\}])^{G} .
$$

This is a filtered algebra (the filtration is induced from the Bernstein filtration on $D(R)$, where $\left.\operatorname{deg} R=\operatorname{deg} R^{*}=1\right)$ and there is a natural epimorphism $\mathbb{C}[M(n, r)] \rightarrow \operatorname{gr} \mathcal{A}_{\lambda}(n, r)$ that is an isomorphism because $\mu$ is flat.

We can also consider the quantization $\mathcal{A}_{\lambda}^{\theta}(n, r)$ of $\mathcal{M}^{\theta}(n, r)$. This is a sheaf (in conical topology) of filtered algebras of $\mathcal{M}^{\theta}(n, r)$ also obtained by quantum Hamiltonian reduction, we will recall how below. Its (derived) global sections coincide with $\mathcal{A}_{\lambda}(n, r)$. So we have the global section functor $\Gamma_{\lambda}^{\theta}: \mathcal{A}_{\lambda}^{\theta}(n, r)$-mod $\rightarrow \mathcal{A}_{\lambda}(n, r)$-mod, where we write $\mathcal{A}_{\lambda}^{\theta}(n, r)$-mod for the category of coherent $\mathcal{A}_{\lambda}^{\theta}(n, r)$-modules and $\mathcal{A}_{\lambda}(n, r)$-mod for the category of finitely generated $\mathcal{A}_{\lambda}(n, r)$-modules.

We note that the variety $\mathcal{M}^{\theta}(n, r)$ comes with another torus action. Namely, let $T_{0}$ denote a maximal torus in $\operatorname{GL}(W)$. The torus $T:=T_{0} \times \mathbb{C}^{\times}$acts on $R$ by $(t, z) .(A, i):=$ $(z A, t i), t \in T_{0}, z \in \mathbb{C}^{\times}, A \in \mathfrak{g l}(V), i \in \operatorname{Hom}(V, W)$. The action naturally lifts to a Hamiltonian action on $T^{*} R$ commuting with $\mathrm{GL}(V)$ and hence descends to $\mathcal{M}^{\theta}(n, r)$. Note that the $T$-action on $\mathcal{M}^{\theta}(n, r)$ commutes with the contracting action of $\mathbb{C}^{\times}$. 
Now let $\overline{\mathcal{A}}_{\lambda}(n, r)$ denote the algebra obtained similarly to $\mathcal{A}_{\lambda}(n, r)$ but for the $G$-module $\bar{R}=\mathfrak{s l}(V) \oplus \operatorname{Hom}(V, W)$. Note that $\mathcal{A}_{\lambda}(n, r)=D(\mathbb{C}) \otimes \overline{\mathcal{A}}_{\lambda}(n, r)$ so most questions about the representation theory of $\mathcal{A}_{\lambda}(n, r)$ reduce to similar questions about $\overline{\mathcal{A}}_{\lambda}(n, r)$.

1.2. Classical cases. There is one case that was studied very extensively in the past fifteen years or so: $r=1$. Here the variety $\mathcal{M}^{\theta}(n, 1)$ is the Hilbert scheme $\operatorname{Hilb}^{n}\left(\mathbb{C}^{2}\right)$ of $n$ points on $\mathbb{C}^{2}$ and $\mathcal{M}(r, n)=\mathbb{C}^{2 n} / \mathfrak{S}_{n}$. The quantization $\overline{\mathcal{A}}_{\lambda}(n, r)$ is the spherical subalgebra in the Rational Cherednik algebra $H_{\lambda}(n)$ for the pair $\left(\mathfrak{h}, \mathfrak{S}_{n}\right)$, where $\mathfrak{h}$ is the reflection representation of $\mathfrak{S}_{n}$, see $\mathrm{GG}$ for details. The representation theory of $\overline{\mathcal{A}}_{\lambda}(n, 1)$ was studied, for example, in [BEG, GS1, GS2, R, [KR, BE, L1, Wi]. In particular, it is known

(1) when (=for which $\lambda$ ) this algebra has finite homological dimension, [BE],

(2) how to classify its finite dimensional irreducible representations, BEG],

(3) how to compute characters of irreducible modules in the so called category $\mathcal{O}, R$,

(4) how to determine the supports of these modules, [Wi],

(5) how to describe the two-sided ideals of $\overline{\mathcal{A}}_{\lambda}(n, 1)$, [L1],

(6) when an analog of the Beilinson-Bernstein localization theorem holds, GS1, KR.

Let us point out that there is an even more classical special case of the algebras $\overline{\mathcal{A}}_{\lambda}(n, r)$ : when $n=1$. In this case $\mathcal{M}^{\theta}(1, r)=\mathbb{C}^{2} \times T^{*} \mathbb{P}^{r-1}$ and $\overline{\mathcal{A}}_{\lambda}(1, r)=D^{\lambda}\left(\mathbb{P}^{r-1}\right)$ (the algebra of $\lambda$-twisted differential operators). (1)-(6) in this case are known and easy.

We will address analogs of $(1),(2),(5),(6)$ for $\overline{\mathcal{A}}_{\lambda}(n, r)$ with general $n, r$. We prove some results towards $(3),(4)$ as well.

1.3. Main results. First, let us give answers to (1) and (6).

Theorem 1.1. The following is true.

(1) The algebra $\overline{\mathcal{A}}_{\lambda}(n, r)$ has finite global dimension if and only if $\lambda$ is not of the form $\frac{s}{m}$, where $1 \leqslant m \leqslant n$ and $-r m<s<0$.

(2) For $\theta=$ det, the abelian localization holds for $\lambda$ (i.e., $\Gamma_{\lambda}^{\theta}$ is an equivalence) if and only if $\lambda$ is not of the form $\frac{s}{m}$, where $1 \leqslant m \leqslant n$ and $s<0$.

In fact, part (2) is a straightforward consequence of (1) and results of McGerty and Nevins, MN2].

Let us proceed to classification of finite dimensional representations.

Theorem 1.2. The algebra $\overline{\mathcal{A}}_{\lambda}(n, r)$ has a finite dimensional representation if and only if $\lambda=\frac{s}{n}$ with $s$ and $n$ coprime and the homological dimension of $\overline{\mathcal{A}}_{\lambda}(n, r)$ is finite. If that is the case, then the category $\overline{\mathcal{A}}_{\lambda}(n, r)-\bmod _{\text {fin }}$ of finite dimensional representations is equivalent to Vect, the category of vector spaces.

Now let us proceed to the description of two-sided ideals.

Theorem 1.3. The following true:

(1) If the algebra $\overline{\mathcal{A}}_{\lambda}(n, r)$ has infinite homological dimension, then it is simple.

(2) Assume that $\overline{\mathcal{A}}_{\lambda}(n, r)$ has finite homological dimension and let $m$ stand for the denominator of $\lambda$ (equal to $+\infty$ if $\lambda$ is not rational). Then there are $\lfloor n / m\rfloor$ proper two-sided ideals in $\overline{\mathcal{A}}_{\lambda}(n, r)$, all of them are prime, and they form a chain $\overline{\mathcal{A}}_{\lambda}(n, r) \supsetneq \mathcal{J}_{\lfloor n / m\rfloor} \supsetneq \ldots \supsetneq \mathcal{J}_{2} \supsetneq \mathcal{J}_{1} \supsetneq\{0\}$. 
Finally, let us explain some partial results on a category $\mathcal{O}$ for $\overline{\mathcal{A}}_{\lambda}^{\theta}(n, r)$, we will recall necessary definitions below in Section 2.2. We use the notation $\mathcal{O}_{\nu}\left(\mathcal{A}_{\lambda}^{\theta}(n, r)\right)$ for this category. Here $\nu$ is a co-character of $T$ that is supposed to be generic meaning that $\mathcal{M}^{\theta}(n, r)^{\nu\left(\mathbb{C}^{\times}\right)}$is finite (in which case, it is in a natural bijection with the set of $r$-multipartitions of $n$ ). The co-character $\nu$ fails to be generic precisely when it lies on a finite union of suitable hyperplanes in $\operatorname{Hom}\left(\mathbb{C}^{\times}, T\right)$, we will describe them explicitly below.

For now, we need to know the following about the category $\mathcal{O}_{\nu}\left(\mathcal{A}_{\lambda}^{\theta}(n, r)\right)$ :

- The category $\mathcal{O}_{\nu}\left(\mathcal{A}_{\lambda}^{\theta}(n, r)\right)$ is a highest weight category so it makes sense to speak about standard objects $\Delta_{\nu}^{\theta}(p)$.

- The labeling set for standard objects is naturally identified with $\mathcal{M}^{\theta}(n, r)^{T}$, i.e., the set of $r$-multipartitions of $n$.

Theorem 1.4. If the denominator of $\lambda$ is bigger than $n$, then the category $\mathcal{O}_{\nu}\left(\mathcal{A}_{\lambda}^{\theta}(n, r)\right)$ is semisimple. If the denominator of $\lambda$ equals $n$, the category $\mathcal{O}_{\nu}\left(\mathcal{A}_{\lambda}^{\theta}(n, r)\right)$ has only one nontrivial block. That block is equivalent to the nontrivial block of $\mathcal{O}_{\nu}\left(\mathcal{A}_{1 / n r}^{\theta}(n r, 1)\right)$ (a.k.a. the category of $B$-equivariant perverse sheaves on $\mathbb{P}^{n r-1}$ ).

In some cases, we can say which simple objects belong to the nontrivial block, we will do this below.

We can also determine the annihilators of the simple modules in the category $\mathcal{O}$. In this paper we treat a special co-character $\nu: \mathbb{C}^{\times} \rightarrow T$ to be called dominant below. Namely, we pick integers $d_{1}, \ldots, d_{r}$ with $d_{1} \gg d_{2} \gg \ldots \gg d_{r}$. Consider $\nu: \mathbb{C}^{\times} \rightarrow T$ given by $t \mapsto\left(\left(t^{d_{1}}, \ldots, t^{d_{r}}\right), t\right)$, it is easy to see that it is generic (in fact, we can just take $\left.d_{i}-d_{i+1}>n\right)$. We also do not consider the case when $\lambda$ is integral, in this case the category was described in [We].

Now take a multipartition $\tau=\left(\tau^{(1)}, \ldots, \tau^{(r)}\right)$. Let $m$ denote the denominator of $\lambda$, we assume that $m>1$. We assume that $\lambda>0$ so that the functor $\Gamma_{\lambda}^{\theta}$ is an equivalence. Divide $\tau^{(1)}$ by $m$ with remainder: $\tau^{(1)}=m \tau^{\prime}+\tau^{\prime \prime}$, where $\tau^{\prime}, \tau^{\prime \prime}$ are partitions with maximal possible $\left|\tau^{\prime}\right|$, and the sum and the multiplication by $m$ are defined component-wise. For example, if $m=3$ and $\tau=(8,6,1)$, then $\tau^{\prime}=\left(1^{2}\right)$ and $\tau^{\prime \prime}=(5,3,1)$.

Theorem 1.5. Assume that $m>1$. Under the assumptions above, the annihilator of $\Gamma\left(L_{\nu}^{\theta}(\tau)\right)$ (where $L_{\nu}^{\theta}(\tau)$ denotes the irreducible module labeled by $\tau$ ) coincides with the ideal $\mathcal{J}_{\left|\tau^{\prime}\right|}$ from Theorem 1.3.

We also give some results towards a computation of the annihilators of $L_{\nu}^{\theta}(\tau)$ for nondominant $\nu$. Namely, for each two adjacent chambers $C, C^{\prime}$ in $\operatorname{Hom}\left(\mathbb{C}^{\times}, T\right)$, there is a cross-walling bijection $\mathfrak{c w}_{C \rightarrow C^{\prime}}^{\lambda}: \mathcal{M}^{\theta}(n, r)^{T} \rightarrow \mathcal{M}^{\theta}(n, r)^{T}$ that preserves the annihilator of the simple modules. We will define this bijection below.

Acknowledgments. I would like to thank Roman Bezrukavnikov, Dmitry Korb, Davesh Maulik, Andrei Okounkov and Nick Proudfoot for stimulating discussions. I also would like to thank Boris Tsvelikhovsky for comments on an earlier version of this text. My work was supported by the NSF under Grant DMS-1501558.

\section{Preliminaries}

2.1. Symplectic resolutions and their quantizations. Let $X_{0}$ be a normal Poisson affine variety equipped with an action of $\mathbb{C}^{\times}$such that the grading on $\mathbb{C}\left[X_{0}\right]$ is positive (meaning that the graded component $\mathbb{C}\left[X_{0}\right]_{i}$ is zero when $i<0$, and $\mathbb{C}\left[X_{0}\right]_{0}=\mathbb{C}$ ) and 
there is a positive integer $d$ such that $\left\{\mathbb{C}\left[X_{0}\right]_{i}, \mathbb{C}\left[X_{0}\right]_{j}\right\} \subset \mathbb{C}\left[X_{0}\right]_{i+j-d}$. By a symplectic resolution of singularities of $X_{0}$ we mean a pair $(X, \rho)$ of

- a smooth symplectic algebraic variety $X$ (with form $\omega$ )

- a morphism $\rho: X \rightarrow X_{0}$ of Poisson varieties that is a projective resolution of singularities.

Below we assume that $(X, \rho)$ is a symplectic resolution of singularities. Besides, we will assume that $(X, \rho)$ is conical meaning that the $\mathbb{C}^{\times}$-action lifts to $X$ in such a way that $\rho$ is equivariant. This $\mathbb{C}^{\times}$-action will be called contracting later on.

Note that $\rho^{*}: \mathbb{C}\left[X_{0}\right] \rightarrow \mathbb{C}[X]$ is an isomorphism because $X_{0}$ is normal. By the GrauertRiemenschneider theorem, we have $H^{i}\left(X, \mathcal{O}_{X}\right)=0$ for $i>0$.

If $X, X^{\prime}$ are two conical symplectic resolutions of $X_{0}$, then the Pickard groups of $X, X^{\prime}$ are naturally identified, see, e.g., [BPW, Proposition 2.19]. Moreover, the Chern class map defines an isomorphism $\mathbb{C} \otimes_{\mathbb{Z}} \operatorname{Pic}(X) \stackrel{\sim}{\rightarrow} H^{2}(X, \mathbb{C})$, see, e.g., [BPW, Section 2.3]. Let us write $\tilde{\mathfrak{p}}$ for $H^{2}(X, \mathbb{C})$ and let $\tilde{\mathfrak{p}}_{\mathbb{Z}}$ denote the image of $\operatorname{Pic}(X)$ in $H^{2}(X, \mathbb{C})$.

Set $\tilde{\mathfrak{p}}_{\mathbb{R}}:=\mathbb{R} \otimes_{\mathbb{Z}} \tilde{\mathfrak{p}}_{\mathbb{Z}}$. According to Namikawa, [Nam, there is a linear hyperplane arrangement in $\tilde{\mathfrak{p}}_{\mathbb{R}}$ together with an action of a crystallographic reflection group $W$ subject to the following conditions:

- The walls for $W$ are in the arrangement.

- The conical symplectic resolutions of $X$ are classified by $W$-conjugacy classes of chambers.

For $\theta$ inside a chamber, we will write $X^{\theta}$ for the corresponding resolution.

We will study quantizations of $X_{0}$ and $X$. By a quantization of $X_{0}$, we mean a filtered algebra $\mathcal{A}$ together with an isomorphism gr $\mathcal{A} \cong \mathbb{C}\left[X_{0}\right]$ of graded Poisson algebras.

By a quantization of $X=X^{\theta}$, we mean a sheaf $\mathcal{A}^{\theta}$ of filtered algebras in the conical topology on $X$ (in this topology, "open" means Zariski open and $\mathbb{C}^{\times}$-stable) that is complete and separated with respect to the filtration together with an isomorphism gr $\mathcal{A}^{\theta} \cong \mathcal{O}_{X^{\theta}}$ (of sheaves of graded Poisson algebras). A result of Bezrukavnikov and Kaledin, BeKa], (with variations given in [L2, Section 2.3]) shows that quantizations $\mathcal{A}^{\theta}$ are parameterized (up to an isomorphism) by the points in $\tilde{\mathfrak{p}}$. We write $\mathcal{A}_{\lambda}^{\theta}$ for the quantization corresponding to $\lambda \in \tilde{\mathfrak{p}}$. Note that $\mathcal{A}_{-\lambda}^{\theta}$ is isomorphic to $\left(\mathcal{A}_{\lambda}^{\theta}\right)^{o p p}$, this follows from [L2, Section 2.3].

We set $\mathcal{A}_{\lambda}=\Gamma\left(\mathcal{A}_{\lambda}^{\theta}\right)$. It follows from [BPW, Section 3.3] that the algebras $\mathcal{A}_{\lambda}$ are independent from the choice of $\theta$. From $H^{i}\left(X^{\theta}, \mathcal{O}_{X^{\theta}}\right)=0$, we deduce that the higher cohomology of $\mathcal{A}_{\lambda}^{\theta}$ vanishes and that $\mathcal{A}_{\lambda}$ is a quantization of $\mathbb{C}[X]=\mathbb{C}\left[X_{0}\right]$. By the previous paragraph, $\mathcal{A}_{-\lambda} \cong \mathcal{A}_{\lambda}^{\text {opp }}$.

Let us compare the categories $\mathcal{A}_{\lambda}$-mod of finitely generated $\mathcal{A}_{\lambda}$-modules and $\operatorname{Coh}\left(\mathcal{A}_{\lambda}^{\theta}\right)$ of coherent sheaves of $\mathcal{A}_{\lambda}^{\theta}$-modules. We have functors $\Gamma: \operatorname{Coh}\left(\mathcal{A}_{\lambda}^{\theta}\right) \rightarrow \mathcal{A}_{\lambda}$-mod of taking global sections and its left adjoint, the localization functor Loc. When we need to indicate the dependence on $(\lambda, \theta)$, we write $\Gamma_{\lambda}^{\theta}, \operatorname{Loc}_{\lambda}^{\theta}$. We say that that $(\lambda, \theta)$ satisfy abelian (resp., derived) localization if the functors $\Gamma_{\lambda}^{\theta}$, $\operatorname{Loc}_{\lambda}^{\theta}$ are mutually inverse (resp., if the corresponding derived functors $R \Gamma_{\lambda}^{\theta}, L \operatorname{Loc}_{\lambda}^{\theta}$ are mutually inverse).

The following result was obtained in [BPW, Section 5.3].

Lemma 2.1. Let $\chi \in \tilde{\mathfrak{p}}_{\mathbb{Z}}$ be ample for $X^{\theta}$ and let $\lambda \in \tilde{\mathfrak{p}}$. Then there $i s n_{0} \in \mathbb{Z}$ such that $\left(\lambda^{\prime}+n \chi, \theta\right)$ satisfies abelian localization for all $n \geqslant n_{0}$. 
2.2. Category $\mathcal{O}$. Suppose that we have a conical symplectic resolution $X=X^{\theta}$ that comes equipped with a Hamiltonian action of a torus $T$ that commutes with the contracting $\mathbb{C}^{\times}$-action. Let $\lambda \in \tilde{\mathfrak{p}}$. The action of $T$ on $\mathcal{O}_{X}$ lifts to a Hamiltonian action of $T$ on $\mathcal{A}_{\lambda}^{\theta}$. So we get a Hamiltonian action on $\mathcal{A}_{\lambda}$. By $\Phi$ we denote the quantum comoment map $\mathfrak{t} \rightarrow \mathcal{A}_{\lambda}$.

Let $\nu: \mathbb{C}^{\times} \rightarrow T$ be a one-parameter subgroup. The subgroup $\nu$ induces a grading $\mathcal{A}_{\lambda}=\bigoplus_{i \in \mathbb{Z}} \mathcal{A}_{\lambda}^{i, \nu}$. We set $\mathcal{A}_{\lambda}^{\geqslant 0, \nu}=\bigoplus_{i \geqslant 0} \mathcal{A}_{\lambda}^{i, \nu}$ and define $\mathcal{A}_{\lambda}^{>0, \nu}$ similarly. Further, set $\mathrm{C}_{\nu}\left(\mathcal{A}_{\lambda}\right):=\mathcal{A}_{\lambda}^{0, \nu} / \bigoplus_{i>0} \mathcal{A}_{\lambda}^{-i, \nu} \mathcal{A}_{\lambda}^{i, \nu}$. Note that $\mathcal{A}_{\lambda} / \mathcal{A}_{\lambda} \mathcal{A}_{\lambda}^{>0, \nu}$ is an $\mathcal{A}_{\lambda} \mathrm{C}_{\nu}\left(\mathcal{A}_{\lambda}\right)$-bimodule, while $\mathcal{A}_{\lambda} / \mathcal{A}_{\lambda}^{<0, \nu} \mathcal{A}_{\lambda}$ is a $\mathrm{C}_{\nu}\left(\mathcal{A}_{\lambda}\right)-\mathcal{A}_{\lambda}$-bimodule.

Define the category $\mathcal{O}_{\nu}\left(\mathcal{A}_{\lambda}\right)$ as the full subcategory of $\mathcal{A}_{\lambda}$-mod consisting of all modules, where the action of $\mathcal{A}_{\lambda}^{>0, \nu}$ is locally nilpotent. We get two functors $\Delta_{\nu}, \nabla_{\nu}$ : $\mathrm{C}_{\nu}\left(\mathcal{A}_{\lambda}\right)-\bmod \rightarrow \mathcal{O}_{\nu}\left(\mathcal{A}_{\lambda}\right)$ given by

$$
\Delta_{\nu}(N):=\left(\mathcal{A}_{\lambda} / \mathcal{A}_{\lambda} \mathcal{A}_{\lambda}^{>0, \nu}\right) \otimes_{\mathrm{C}_{\nu}\left(\mathcal{A}_{\lambda}\right)} N, \nabla_{\nu}(N):=\operatorname{Hom}_{\mathrm{C}_{\nu}\left(\mathcal{A}_{\lambda}\right)}\left(\mathcal{A}_{\lambda} / \mathcal{A}_{\lambda}^{<0, \nu} \mathcal{A}_{\lambda}, N\right) .
$$

Here we consider the restricted Hom (with respect to the natural grading on $\mathcal{A}_{\lambda} / \mathcal{A}_{\lambda}^{<0, \nu} \mathcal{A}_{\lambda}$ ).

Now suppose that $\left|X^{T}\right|<\infty$. We say that a one-parameter group $\nu: \mathbb{C}^{\times} \rightarrow T$ is generic if $X^{\nu\left(\mathbb{C}^{\times}\right)}=X^{T}$. Equivalently, $\nu$ is generic if and only if it does not lie in ker $\kappa$ for any character $\kappa$ of the $T$-action on $\bigoplus_{p \in X^{T}} T_{p} X$. The hyperplanes ker $\kappa$ split the lattice $\operatorname{Hom}\left(\mathbb{C}^{\times}, T\right)$ into the union of polyhedral regions to be called chambers (of one-parameter subgroups).

Suppose that $\nu$ is generic. Further, pick a generic element $\theta \in \tilde{\mathfrak{p}}_{\mathbb{Z}}$ with $X=X^{\theta}$ and $\lambda_{0} \in \tilde{\mathfrak{p}}$. Let $\lambda:=\lambda_{0}+n \theta$ for $n \gg 0$. The following results were obtained in [BLPW] and [L5], see [L7, Proposition 4.1] for precise references.

Proposition 2.2. The following is true:

(1) The category $\mathcal{O}_{\nu}\left(\mathcal{A}_{\lambda}\right)$ only depends on the chamber of $\nu$.

(2) The natural functor $D^{b}\left(\mathcal{O}_{\nu}\left(\mathcal{A}_{\lambda}\right)\right) \rightarrow D^{b}\left(\mathcal{A}_{\lambda}\right.$-mod) is a full embedding.

(3) $\mathrm{C}_{\nu}\left(\mathcal{A}_{\lambda}\right)=\mathbb{C}\left[X^{T}\right]$.

(4) More generally, we have $\mathrm{C}_{\nu_{0}}\left(\mathcal{A}_{\lambda}\right)=\bigoplus_{Z} \mathcal{A}_{\iota_{Z}^{*}(\lambda)-\rho_{Z}}^{Z}$, where the summation is taken over the irreducible components $Z$ of $X^{\nu_{0}\left(\mathbb{C}^{\times}\right)}, \iota_{Z}$ is the embedding $Z \hookrightarrow X, \iota_{Z}^{*}$ : $H^{2}(X, \mathbb{C}) \rightarrow H^{2}(Z, \mathbb{C})$ is the corresponding pull-back map, $\rho_{Z}$ equals to a half of the 1 st Chern class of the contracting bundle of $Z$, and $\mathcal{A}_{\iota_{Z}^{*}(\lambda)-\rho_{Z}}^{Z}$ stands for the global sections of the filtered quantization of $Z$ with period $\iota_{Z}^{*}(\lambda)-\rho_{Z}$.

(5) The category $\mathcal{O}_{\nu}\left(\mathcal{A}_{\lambda}\right)$ is highest weight, where the standard objects are $\Delta_{\nu}(p)$, the costandard objects are $\nabla_{\nu}(p)$, where $p \in X^{T}$. For an order, which is a part of the definition of a highest weight structure, we take the contraction order on $X^{T}$ given by $\nu$.

(6) Suppose $\nu_{0}$ lies in the face of a chamber containing $\nu$. Then $\Delta_{\nu_{0}}, \nabla_{\nu_{0}}$ restrict to exact functors $\mathcal{O}_{\nu}\left(\mathrm{C}_{\nu_{0}}\left(\mathcal{A}_{\lambda}\right)\right) \rightarrow \mathcal{O}_{\nu}\left(\mathcal{A}_{\lambda}\right)$. Moreover, $\Delta_{\nu}=\Delta_{\nu_{0}} \circ \triangleq$ and $\nabla_{\nu}=$ $\nabla_{\nu_{0}} \circ \underline{\nabla}$, where we write $\underline{\Delta}$ and $\underline{\nabla}$ are the standardization and costandardization functors for $\mathcal{O}_{\nu}\left(\mathrm{C}_{\nu_{0}}\left(\mathcal{A}_{\lambda}\right)\right)$.

Let us explain what we mean by the contracting bundle in (4). This is the subvariety in $X$ consisting of all points $x$ such that $\lim _{t \rightarrow 0} \nu_{0}(t) x$ exists and lies in $Z$. Highest weight categories mentioned in (5) will be recalled in the next section.

Now let us mention the holonomicity property, see [L5, Section 4.4] and [L6, Theorems $1.2,1.3]$. 
Lemma 2.3. Every module from category $\mathcal{O}_{\nu}\left(\mathcal{A}_{\lambda}\right)$ is holonomic in the sense of [L6]. In particular, if $M$ is a simple object in $\mathcal{O}_{\nu}\left(\mathcal{A}_{\lambda}\right)$, then $\mathrm{GK}-\operatorname{dim} M=\frac{1}{2} \mathrm{GK}-\operatorname{dim}\left(\mathcal{A}_{\lambda} / \operatorname{Ann} M\right)$.

We will also need the following important property, [BLPW, Lemma 6.4].

Lemma 2.4. The classes of standard and costandard objects in $K_{0}\left(\mathcal{O}_{\nu}\left(\mathcal{A}_{\lambda}^{\theta}\right)\right)$ coincide.

2.3. Standardly stratified structures and cross-walling functors. Let us start by recalling standardly stratified categories following [LW].

Let $\mathcal{C}$ be a $\mathbb{C}$-linear abelian category equivalent to the category of finite dimensional representations of a finite dimensional algebra. Let $\mathcal{T}$ be an indexing set of the simple objects in $\mathcal{C}$. We write $L(\tau), P(\tau)$ for the simple and indecomposable projective objects indexed by $\tau \in \mathcal{T}$. The additional structure of a standardly stratified category on $\mathcal{C}$ is a partial pre-order $\leqslant$ on $\mathcal{T}$ that should satisfy certain axioms to be explained below. Let us write $\Xi$ for the set of equivalence classes of $\leqslant$, this is a poset (with partial order again denoted by $\leqslant)$ that comes with a natural surjection $\varrho: \mathcal{T} \rightarrow \Xi$. The pre-order $\leqslant$ defines a filtration on $\mathcal{C}$ by Serre subcategories indexed by $\Xi$. Namely, to $\xi \in \Xi$ we assign the subcategories $\mathcal{C}_{\leqslant \xi}$ that is the Serre span of the simples $L(\tau)$ with $\varrho(\tau) \leqslant \xi$. Define $\mathcal{C}_{<\xi}$ analogously and let $\mathcal{C}_{\xi}$ denote the quotient $\mathcal{C}_{\leqslant \xi} / \mathcal{C}_{\xi}$. Let $\pi_{\xi}$ denote the quotient functor $\mathcal{C}_{\leqslant \xi} \rightarrow \mathcal{C}_{\xi}$. Let us write $\Delta_{\xi}: \mathcal{C}_{\xi} \rightarrow \mathcal{C}_{\leqslant \xi}$ for the left adjoint functor of $\pi_{\xi}$. Also we write gr $\mathcal{C}$ for $\bigoplus_{\xi} \mathcal{C}_{\xi}, \Delta$ for $\bigoplus_{\xi} \Delta_{\xi}:$ gr $\mathcal{C} \rightarrow \mathcal{C}$. We call $\Delta$ the standardization functor. Finally, for $\tau \in \varrho^{-1}(\xi)$ we write $L_{\xi}(\tau)$ for $\pi_{\xi}(L(\tau)), P_{\xi}(\tau)$ for the projective cover of $L_{\xi}(\tau)$ in $\mathcal{C}_{\xi}$ and $\Delta(\tau)$ for $\Delta_{\xi}\left(P_{\xi}(\tau)\right)$. The object $\Delta(\tau)$ is called standard. The object $\bar{\Delta}(\tau):=\Delta_{\xi}\left(L_{\xi}(\tau)\right)$ is called proper standard. Note that there is a natural epimorphism $P(\tau) \rightarrow \Delta(\tau)$.

The axioms to be satisfied by $(\mathcal{C}, \leqslant)$ in order to give a standardly stratified structure are as follows.

(SS1) The functor $\Delta: \operatorname{gr} \mathcal{C} \rightarrow \mathcal{C}$ is exact.

(SS2) The projective $P(\tau)$ admits an epimorphism onto $\Delta(\tau)$ whose kernel has a filtration with successive quotients $\Delta\left(\tau^{\prime}\right)$, where $\tau^{\prime}>\tau$.

When $\mathcal{C}_{\xi}=$ Vect for all $\xi$ (in which case $\Xi=\mathcal{T}$ ), we recover the classical notion of a highest weight category.

Note that $\mathcal{C}^{o p p}$ is also a standardly stratified category with the same poset $\Xi$, [LW, Section 2.2]. So we have the exact costandardization functor $\nabla_{\xi}$, the right adjoint of $\pi_{\xi}$.

Let us describe a standardly stratified structure on $\mathcal{O}_{\nu}\left(\mathcal{A}_{\lambda}\right)$ (where $\lambda$ is as in Proposition 2.2) that comes from a one-parameter subgroup $\nu_{0}$ lying in a face of the chamber containing $\nu$. Then $\nu_{0}$ defines the order on the set of irreducible components of $X^{\nu_{0}\left(\mathbb{C}^{\times}\right)}$ (by contraction, see [L5, Section 6.1] for details). So we get a pre-order $\leqslant \nu_{0}$ on the set $X^{T}$. It is easy to see (and was checked in [L5, Section 6.1]) that the order $\leqslant \nu$ refines $\leqslant \nu_{0}$.

The following proposition is the main result of [L5, Section 6].

Proposition 2.5. Let $\lambda$ be as in Proposition 2.2. Then the pre-order $\leqslant \nu_{0}$ defines a standardly stratified structure on $\mathcal{O}_{\nu}\left(\mathcal{A}_{\lambda}\right)$. The associated graded category is $\mathcal{O}_{\nu}\left(\mathrm{C}_{\nu_{0}}\left(\mathcal{A}_{\lambda}\right)\right)$. The standardization functor is $\Delta_{\nu_{0}}$ and the costandardization functor is $\nabla_{\nu_{0}}$.

Let us now discuss cross-walling functors that are derived equivalences between categories $\mathcal{O}$ corresponding to different generic one-parameter subgroups. Namely, let $\nu, \nu^{\prime}$ be two generic one-parameter subgroups and $\lambda$ be as in Proposition 2.2. The following proposition is established in [L5, Section 7].

Proposition 2.6. The following is true: 
(1) There is an equivalence $\mathfrak{C W}_{\nu^{\prime} \leftarrow \nu}: D^{b}\left(\mathcal{O}_{\nu}\left(\mathcal{A}_{\lambda}\right)\right) \rightarrow D^{b}\left(\mathcal{O}_{\nu^{\prime}}\left(\mathcal{A}_{\lambda}\right)\right)$ such that we have a bifunctorial isomorphism

$$
\operatorname{Hom}_{D^{b}\left(\mathcal{O}_{\nu^{\prime}}\left(\mathcal{A}_{\lambda}\right)\right)}\left(\mathfrak{C W}_{\nu^{\prime} \leftarrow \nu}(M), N\right) \stackrel{\sim}{\rightarrow} \operatorname{Hom}_{D^{b}\left(\mathcal{A}_{\lambda} \text {-mod }\right)}(M, N),
$$

where $M \in D^{b}\left(\mathcal{O}_{\nu}\left(\mathcal{A}_{\lambda}\right)\right), N \in D^{b}\left(\mathcal{O}_{\nu^{\prime}}\left(\mathcal{A}_{\lambda}\right)\right)$.

(2) Suppose that $\nu_{0}$ lies in a common face of the chambers containing $\nu, \nu^{\prime}$. Then we have an isomorphism $\mathfrak{C W}_{\nu^{\prime} \leftarrow \nu} \circ \Delta_{\nu_{0}} \stackrel{\sim}{\rightarrow} \Delta_{\nu_{0}} \circ \underline{\mathfrak{C}}_{\nu^{\prime} \leftarrow \nu}$, where we write $\underline{\mathfrak{C W}}_{\nu^{\prime} \leftarrow \nu}$ for the cross-walling functor $D^{b}\left(\mathcal{O}_{\nu}\left(\mathrm{C}_{\nu_{0}}\left(\mathcal{A}_{\lambda}\right)\right)\right) \rightarrow D^{b}\left(\mathcal{O}_{\nu^{\prime}}\left(\mathrm{C}_{\nu_{0}}\left(\mathcal{A}_{\lambda}\right)\right)\right)$.

(3) The functor $\mathfrak{C W}_{-\nu \leftarrow \nu}\left[-\frac{1}{2} \operatorname{dim} X\right]$ is the Ringel duality functor, i.e., an equivalence that maps $\Delta_{\nu}(p)$ to $\nabla_{-\nu}(p)$ for all $p \in X^{T}$.

2.4. Wall-crossing functors and bijections. Let $\theta, \theta^{\prime}$ be two generic elements of $\tilde{\mathfrak{p}}_{\mathbb{Z}}$ and $\lambda \in \tilde{\mathfrak{p}}$. Following [BPW, Section 6.3], we are going to produce a derived equivalence $\mathfrak{W C}_{\theta^{\prime} \leftarrow \theta}: D^{b}\left(\operatorname{Coh}\left(\mathcal{A}_{\lambda}^{\theta}\right)\right) \stackrel{\sim}{\rightarrow} D^{b}\left(\operatorname{Coh}\left(\mathcal{A}_{\lambda}^{\theta^{\prime}}\right)\right)$ assuming abelian localization holds for $(\lambda, \theta)$ and derived localization holds for $\left(\lambda, \theta^{\prime}\right)$. Then we set $\mathfrak{W J C}_{\theta^{\prime} \leftarrow \theta}:=L \operatorname{Loc}_{\lambda}^{\theta^{\prime}} \circ \Gamma_{\lambda}^{\theta}$. Note that this functor is right t-exact.

We can give a different realization of $\mathfrak{W C}_{\theta^{\prime} \leftarrow \theta}$. Namely, pick $\lambda^{\prime} \in \lambda+\tilde{\mathfrak{p}}_{\mathbb{Z}}$ such that abelian localization holds for $\left(\lambda^{\prime}, \theta^{\prime}\right)$. We identify $\operatorname{Coh}\left(\mathcal{A}_{\lambda}^{\theta}\right)$ with $\mathcal{A}_{\lambda}$-mod by means of $\Gamma_{\lambda}^{\theta}$ and $\operatorname{Coh}\left(\mathcal{A}_{\lambda}^{\theta^{\prime}}\right)$ with $\mathcal{A}_{\lambda^{\prime}}$-mod by means of $\Gamma_{\lambda^{\prime}}^{\theta^{\prime}}\left(\mathcal{A}_{\lambda, \lambda^{\prime}-\lambda}^{\theta^{\prime}} \otimes_{\mathcal{A}_{\lambda}^{\theta^{\prime}}} \bullet\right)$, where $\mathcal{A}_{\lambda, \lambda^{\prime}-\lambda}^{\theta^{\prime}}$ is the $\mathcal{A}_{\lambda^{\prime}}^{\theta^{\prime}}-\mathcal{A}_{\lambda}^{\theta^{\prime}}$-bimodule quantizing the line bundle corresponding to $\lambda^{\prime}-\lambda$ on $X^{\theta^{\prime}}$. Under these identifications, the functor $\mathfrak{W C}_{\theta^{\prime} \leftarrow \theta}$ becomes $\mathfrak{W C}_{\lambda^{\prime} \leftarrow \lambda}:=\mathcal{A}_{\lambda, \lambda^{\prime}-\lambda}^{(\theta)} \otimes_{\mathcal{A}_{\lambda}}^{L} \bullet$, see [BPW], Section 6.4]. Here we write $\mathcal{A}_{\lambda, \lambda^{\prime}-\lambda}^{(\theta)}$ for the global sections of $\mathcal{A}_{\lambda, \lambda^{\prime}-\lambda}^{\theta}$.

We note that the functor $\mathfrak{W C}_{\lambda^{\prime} \leftarrow \lambda}$ restricts to an equivalence $D^{b}\left(\mathcal{O}_{\nu}\left(\mathcal{A}_{\lambda}\right)\right) \rightarrow D^{b}\left(\mathcal{O}_{\nu}\left(\mathcal{A}_{\lambda^{\prime}}\right)\right)$, see [BLPW, Section 8.1].

We are especially interested in the situation when $\theta, \theta^{\prime}$ lie in the opposite chambers. In this case the long wall-crossing functor $\mathfrak{W C}_{\lambda^{\prime} \leftarrow \lambda}$ is perverse in the sense of Chuang and Rouquier, see [L7, Section 3]. Let us recall the general definition.

Let us recall the general definition. Let $\mathcal{T}^{1}, \mathcal{T}^{2}$ be triangulated categories equipped with $t$-structures that are homologically finite. Let $\mathcal{C}^{1}, \mathcal{C}^{2}$ denote the hearts of $\mathcal{T}^{1}, \mathcal{T}^{2}$, respectively.

We are going to define a perverse equivalence with respect to filtrations $\mathcal{C}^{i}=\mathcal{C}_{0}^{i} \supset \mathcal{C}_{1}^{i} \supset$ $\ldots \supset \mathcal{C}_{k}^{i}=\{0\}$ by Serre subcategories. By definition, this is a triangulated equivalence $\mathcal{F}: \mathcal{T}^{1} \rightarrow \mathcal{T}^{2}$ subject to the following conditions:

(P1) For any $j$, the equivalence $\mathcal{F}$ restricts to an equivalence $\mathcal{T}_{\mathcal{C}_{j}^{1}}^{1} \rightarrow \mathcal{T}_{\mathcal{C}_{j}^{2}}^{2}$, where we write $\mathcal{T}_{\mathcal{C}_{j}^{i}}^{i}, i=1,2$, for the category of all objects in $\mathcal{T}^{i}$ with homology (computed with respect to the t-structures of interest) in $\mathcal{C}_{j}^{i}$.

(P2) For $M \in \mathcal{C}_{j}^{1}$, we have $H_{\ell}(\mathcal{F} M)=0$ for $\ell<j$ and $H_{\ell}(\mathcal{F} M) \in \mathcal{C}_{j+1}^{2}$ for $\ell>j$.

(P3) The functor $M \mapsto H_{j}(\mathcal{F} M)$ induces an equivalence $\mathcal{C}_{j}^{1} / \mathcal{C}_{j+1}^{1} \stackrel{\sim}{\rightarrow} \mathcal{C}_{j}^{2} / \mathcal{C}_{j+1}^{2}$ of abelian categories.

Lemma 2.7. The equivalence $\mathfrak{W C C}_{\lambda^{\prime} \leftarrow \lambda}: D^{b}\left(\mathcal{O}_{\nu}\left(\mathcal{A}_{\lambda}\right)\right) \rightarrow D^{b}\left(\mathcal{O}_{\nu}\left(\mathcal{A}_{\lambda^{\prime}}\right)\right)$ is perverse with $\mathcal{C}_{j}^{1}=\left\{M \in \mathcal{O}_{\nu}\left(\mathcal{A}_{\lambda}\right) \mid \mathrm{GK}-\operatorname{dim} M \leqslant \operatorname{dim} X / 2-j\right\}$ (and $\mathcal{C}_{j}^{2}$ defined similarly).

Now let us discuss the wall-crossing bijections. (P3) gives rise to a bijection $\operatorname{Irr}\left(\mathcal{C}^{1}\right) \rightarrow$ $\operatorname{Irr}\left(\mathcal{C}^{2}\right)$ that is called a wall-crossing bijection and denoted by $\mathfrak{w c}_{\lambda^{\prime} \leftarrow \lambda}$ when we deal with the wall-crossing functors $\mathfrak{W C}_{\lambda^{\prime} \leftarrow \lambda}$. 
Finally, let us recall the following important property of $\mathfrak{W J C}_{\lambda^{\prime} \leftarrow \lambda}$ established in [L5, Section 7.3].

Lemma 2.8. In the case when $\theta=-\theta^{\prime}$, the functor $\mathfrak{W J C}_{\lambda^{\prime} \leftarrow \lambda}: D^{b}\left(\mathcal{O}_{\nu}\left(\mathcal{A}_{\lambda}\right)\right) \rightarrow D^{b}\left(\mathcal{O}_{\nu}\left(\mathcal{A}_{\lambda^{\prime}}\right)\right)$ is a Ringel duality functor.

\section{Gieseker varieties AND their QuAntizations}

The definition of the Gieseker varieties $\mathcal{M}(n, r), \mathcal{M}^{\theta}(n, r)$ (for $\theta=\operatorname{det} \operatorname{or} \operatorname{det}^{-1}$ ) was recalled in the introduction. We note that $\mathcal{M}^{\theta}(n, r), \mathcal{M}^{-\theta}(n, r)$ are sympletomorphic via the isomorphism induced by $(A, B, i, j) \mapsto\left(B^{*},-A^{*}, j^{*},-i^{*}\right)$.

We also consider the varieties $\overline{\mathcal{M}}(n, r), \overline{\mathcal{M}}^{\theta}(n, r)$ that are obtained similarly but with the space $R=\operatorname{End}(V) \oplus \operatorname{Hom}(V, W)$ replaced with $\mathfrak{s l}(V) \oplus \operatorname{Hom}(V, W)$. So $\mathcal{M}(n, r)=$ $\mathbb{C}^{2} \times \overline{\mathcal{M}}(n, r)$ and $\mathcal{M}^{\theta}(n, r)=\mathbb{C}^{2} \times \overline{\mathcal{M}}^{\theta}(n, r)$.

3.1. Quantizations. Let us discuss some questions about the quantizations $\overline{\mathcal{A}}_{\lambda}^{\theta}(n, r)$ of $\overline{\mathcal{M}}^{\theta}(n, r)$. These quantizations are defined via

$$
\overline{\mathcal{A}}_{\lambda}^{\theta}(n, r)=\left[D(\bar{R}) /\left.D(\bar{R})\left\{x_{R}-\lambda \operatorname{tr}(x) \mid x \in \mathfrak{g}\right\}\right|_{\left(T^{*} \bar{R}\right)^{\theta-s s}}\right]^{G}
$$

Recall that $\mathcal{A}_{\lambda}(n, r)=D(\mathbb{C}) \otimes \overline{\mathcal{A}}_{\lambda}(n, r)$. Let $t$ be the coordinate on $\mathbb{C}$. Then the categories $\mathcal{O}_{\nu}\left(\mathcal{A}_{\lambda}(n, r)\right)$ and $\mathcal{O}_{\nu}\left(\overline{\mathcal{A}}_{\lambda}(n, r)\right)$ are equivalent: a functor $\mathcal{O}_{\nu}\left(\overline{\mathcal{A}}_{\lambda}(n, r)\right) \rightarrow \mathcal{O}_{\nu}\left(\mathcal{A}_{\lambda}(n, r)\right)$ is given by $M \mapsto \mathbb{C}[t] \otimes M$ and a quasi-inverse functor sends $N$ to the annihilator of $\partial_{t}$.

Now let us discuss periods. By [L2, Section 5], the period of $\overline{\mathcal{A}}_{\lambda}^{\theta}(n, r)$ is $\lambda-\rho$, where $\varrho$ is half the character of the action of $\operatorname{GL}(n)$ on $\Lambda^{\text {top }} R$. So $\varrho=-r /$ and the period of $\overline{\mathcal{A}}_{\lambda}^{\theta}(n, r)$ is $\lambda+r / 2$.

So we have

$$
\overline{\mathcal{A}}_{\lambda}(n, r)^{o p p} \cong \overline{\mathcal{A}}_{-\lambda-r}(n, r) .
$$

Lemma 3.1. We have $\overline{\mathcal{A}}_{\lambda}(n, r) \cong \overline{\mathcal{A}}_{-\lambda-r}(n, r)$.

Proof. Recall, Section 2.1 , that $\overline{\mathcal{A}}_{\lambda}(n, r) \stackrel{\sim}{\rightarrow} \Gamma\left(\overline{\mathcal{A}}_{\lambda}^{ \pm \theta}(n, r)\right)$. We have produced a symplectomorphism $\iota: \overline{\mathcal{M}}^{\theta}(n, r) \stackrel{\sim}{\rightarrow} \overline{\mathcal{M}}^{-\theta}(n, r)$ via $(A, B, i, j) \rightarrow\left(B^{*},-A^{*}, j^{*},-i^{*}\right)$. On the second cohomology this isomorphism induces the multiplication by -1 . It follows that $\Gamma\left(\overline{\mathcal{A}}_{\lambda}^{\theta}(n, r)\right) \cong \Gamma\left(\overline{\mathcal{A}}_{-\lambda-r}^{-\theta}(n, r)\right)$ and our claim follows.

We conclude that $\overline{\mathcal{A}}_{\lambda}(n, r)^{\text {opp }} \cong \overline{\mathcal{A}}_{\lambda}(n, r)$.

Now let us discuss derived localization and wall-crossing bimodules. The following is a special case of the main result of [MN1.

Lemma 3.2. Derived localization holds for $(\lambda, \theta)$ if and only if $\overline{\mathcal{A}}_{\lambda}(n, r)$ has finite homological dimension.

3.2. Fixed point subvarieties and their quantizations. Recall that the torus $T=$ $\mathbb{C}^{\times} \times T_{0}$, where $T_{0}$ is a maximal torus in $\operatorname{GL}(r)$, acts on $\mathcal{M}^{\theta}(n, r)$.

Now want to understand the structure of $\mathcal{M}^{\theta}(n, r)^{\nu_{0}\left(\mathbb{C}^{\times}\right)}$for a non-generic $\nu_{0}$ that lies in the interior of a codimension 1 face in a chamber. Let $\nu_{0}(t)=\left(\operatorname{diag}\left(t^{d_{1}}, \ldots, t^{d_{r}}\right), t^{k}\right)$. The following claim follows directly from the description of the tangent space in the fixed points given in [NY, Theorem 3.2].

Lemma 3.3. The walls in the lattice of one-parameter subgroups are given by $k=0$ and $d_{i}-d_{j}=$ sk with $s \in \mathbb{Z}$ such that $|s|<n$. 
The description of $\mathcal{M}^{\theta}(n, r)^{\nu_{0}\left(\mathbb{C}^{\times}\right)}$for $\nu_{0}$ with $k=0$ is classical.

Lemma 3.4. For $k=0$ (and pairwise different $d_{1}, \ldots, d_{r}$ ), we have

$$
\mathcal{M}^{\theta}(n, r)^{\nu_{0}\left(\mathbb{C}^{\times}\right)}=\bigsqcup_{n_{1}+\ldots+n_{r}=n} \prod_{i=1}^{r} \mathcal{M}^{\theta}\left(n_{i}, 1\right)
$$

Recall that $\mathcal{M}^{\theta}\left(n_{i}, 1\right)=\operatorname{Hilb}_{n_{i}}\left(\mathbb{C}^{2}\right)$ (when $n_{i}=0$, we assume that this variety is a single point). Lemma 3.4 gives an identification of $\mathcal{M}^{\theta}(n, r)^{T}$ with the set of $r$-multipartitions of $n$. Indeed, the fixed points of $\mathbb{C}^{\times}$in $\mathcal{M}^{\theta}\left(n_{i}, 1\right)$ are in a one-to-one correspondence with the partitions of $n_{i}$.

The description for $s \neq 0$ is less classical but is also known. Using an analog of the previous lemma, this description easily reduces to the case when $r=2$. Here the components of the fixed points are quiver varieties for finite type A Dynkin diagrams. We will not need a more precise description in this paper.

Now, for $\nu_{0}(t)=\left(\operatorname{diag}\left(t^{d_{1}}, \ldots, t^{d_{r}}\right), 1\right)$ with $d_{1} \gg d_{2} \gg \ldots . \gg d_{r}$, we want to describe the sheaves $\mathrm{C}_{\nu_{0}}\left(\mathcal{A}_{\lambda}^{\theta}(n, r)\right)$.

Proposition 3.5. For the irreducible component $Z$ of $\mathcal{M}^{\theta}(n, r)^{\nu_{0}\left(\mathbb{C}^{\times}\right)}$corresponding to the $r$-multipartition $\left(n_{1}, \ldots, n_{r}\right)$ of $n$, we have $\left.\mathrm{C}_{\nu_{0}}\left(\mathcal{A}_{\lambda}^{\theta}(n, r)\right)\right|_{Z}=\bigotimes_{i=1}^{r} \mathcal{A}_{\lambda+i-1}^{\theta}\left(n_{i}, 1\right)$ (the ith factor is absent if $\left.n_{i}=0\right)$. Moreover, for a Zariski generic $\lambda \in \mathbb{C}$, we have $\mathrm{C}_{\nu_{0}}\left(\mathcal{A}_{\lambda}(n, r)\right)=$ $\bigoplus \bigotimes_{i=1}^{r} \mathcal{A}_{\lambda+(i-1)}\left(n_{i}, r\right)$, where the summation is taken over all $r$-multipartitions $\left(n_{1}, \ldots, n_{r}\right)$.

Proof. Let $\mu=\left(n_{1}, \ldots, n_{r}\right), Z_{\mu}$ denote the corresponding component of $X^{\nu_{0}\left(\mathbb{C}^{\times}\right)}$. Let $Y_{\mu}$ denote the contracting vector bundle for $Z_{\mu}$. According to (4) of Proposition 2.2, we need to compute the Chern character of $Y_{\mu}$.

First, consider the following situation. Set $V:=\mathbb{C}^{n}, W=\mathbb{C}^{r}$. Choose a decomposition $W=W^{1} \oplus W^{2}$ with $\operatorname{dim} W^{i}=r_{i}$ and consider the one-parameter subgroup $\alpha$ of $\mathrm{GL}(W)$ acting trivially on $W^{2}$ and by $t \mapsto t$ on $W^{1}$. The components of the fixed points in $\mathcal{M}^{\theta}(n, r)$ are in one-to-one correspondence with decompositions on $n$ into the sum of two parts. Pick such a decomposition $n=n_{1}+n_{2}$ and consider the splitting $V=V^{1} \oplus V^{2}$ into the sum of two spaces of the corresponding dimensions. Then the corresponding component $Z^{\prime} \subset \mathcal{M}^{\theta}(n, r)^{\alpha\left(\mathbb{C}^{\times}\right)}$is $\mathcal{M}^{\theta}\left(n_{1}, r_{1}\right) \times \mathcal{M}^{\theta}\left(n_{2}, r_{2}\right)$.

Nakajima has described the contracting bundle $Y \rightarrow Z^{\prime}$, this is basically in [Nak, Proposition 3.13]. This is the bundle on $Z^{\prime}=\mathcal{M}^{\theta}\left(n_{1}, r_{1}\right) \times \mathcal{M}^{\theta}\left(n_{2}, r_{2}\right)$ that descends from the $\operatorname{GL}\left(n_{1}\right) \times \mathrm{GL}\left(n_{2}\right)$-module $\operatorname{ker} \beta^{12} / \operatorname{im} \alpha^{12}$, where $\alpha^{12}, \beta^{12}$ are certain $\operatorname{GL}\left(n_{1}\right) \times \operatorname{GL}\left(n_{2}\right)$ equivariant linear maps

$$
\operatorname{Hom}\left(V^{2}, V^{1}\right) \stackrel{\alpha^{12}}{\longrightarrow} \operatorname{Hom}\left(V^{2}, V^{1}\right)^{\oplus 2} \oplus \operatorname{Hom}\left(W^{2}, V^{1}\right) \oplus \operatorname{Hom}\left(V^{2}, W^{1}\right) \stackrel{\beta^{12}}{\longrightarrow} \operatorname{Hom}\left(V^{2}, V^{1}\right) .
$$

We do not need to know the precise form of the maps $\alpha^{12}, \beta^{12}$, what we need is that $\alpha^{12}$ is injective while $\beta^{12}$ is surjective. So $\operatorname{ker} \beta^{12} / \operatorname{im} \alpha^{12} \cong \operatorname{Hom}\left(W^{2}, V^{1}\right) \oplus \operatorname{Hom}\left(V^{2}, W^{1}\right)$, an isomorphism of $\mathrm{GL}\left(n_{1}\right) \times \mathrm{GL}\left(n_{2}\right)$-modules.

It is easy to see that if $\alpha^{\prime}: \mathbb{C}^{\times} \rightarrow \mathrm{GL}\left(r_{1}\right)$ is a homomorphism of the form $t \mapsto$ $\operatorname{diag}\left(t^{d_{1}}, \ldots, t^{d_{k}}\right)$ with $d_{1}, \ldots, d_{k} \gg 0$, then the contracting bundle for the one-parametric subgroup $\left(\alpha^{\prime}, 1\right): \mathbb{C}^{\times} \rightarrow \mathrm{GL}\left(W^{1}\right) \times \mathrm{GL}\left(W^{2}\right)$ coincides with the sum of the contracting bundles for $\alpha^{\prime}$ and for $\alpha$. So we see inductively that the vector bundle $Y_{\mu}$ on $Z_{\mu}$ descends from the $\prod_{i=1}^{r} \operatorname{GL}\left(n_{i}\right)$-module $\sum_{i=1}^{r}\left(\left(\mathbb{C}^{n_{i}}\right)^{\oplus r-i} \oplus\left(\mathbb{C}^{n^{*} *}\right)^{\oplus i-1}\right)$. Therefore the 1st Chern class of $Y_{\mu}$ is $\sum_{i=1}^{r}(r+1-2 i) c_{i}$, where we write $c_{i}$ for the generator of $H^{2}\left(\mathcal{M}^{\theta}\left(n_{i}, 1\right)\right)$ (that is the first Chern class of $\mathcal{O}(1)$ ). 
Now to prove the claim of this proposition we will use (4) of Proposition 2.2. The map $\iota_{Z}^{*}$ sends the generator $c$ of $H^{2}\left(\mathcal{M}^{\theta}(n, r)\right)$ to $\sum_{i=1}^{r} c_{i}$. The period of $\mathcal{A}_{\lambda}^{\theta}(n, r)$ is $(\lambda+r / 2) c$. So the period of $\mathcal{A}_{\lambda+i-1}^{\theta}\left(n_{i}, 1\right)$ is $(\lambda+1 / 2+i-1) c_{i}$. Now the statement of this proposition is a direct corollary of (4) of Proposition 2.2.

3.3. Symplectic leaves and slices. Here we want to describe the symplectic leaves of $\overline{\mathcal{M}}(n, r)$ and study the structure of the variety near a symplectic leaf.

Lemma 3.6. The point 0 is a single leaf of $\overline{\mathcal{M}}(n, r)$.

Proof. It is enough to show that the maximal ideal of 0 in $\mathbb{C}\left[T^{*} \bar{R}\right]^{G}$ is Poisson. Since $\bar{R}$ does not include the trivial $G$-module as a direct summand, we see that all homogeneous elements in $\mathbb{C}\left[T^{*} \bar{R}\right]^{G}$ have degree 2 or higher. It follows that the bracket of any two homogeneous elements also has degree 2 or higher and our claim is proved.

Now let us describe the slices to symplectic leaves in $\mathcal{M}(n, r)$. When $r=1$, then $\mathcal{M}(n, r)=\mathbb{C}^{2 n} / S_{n}$ and the description is easy.

Proposition 3.7. Let $r>1$. Then the following is true:

(1) The symplectic leaves of $\overline{\mathcal{M}}(n, r)$ are parameterized by the unordered collections of numbers $\left(n_{1}, \ldots, n_{k}\right)$ with $\sum_{i=1}^{k} n_{i} \leqslant n$.

(2) There is a transversal slice to the leaf as above that is isomorphic to the formal neighborhood of 0 in $\prod_{i=1}^{k} \overline{\mathcal{M}}\left(n_{i}, r\right)$.

Proof. Pick $x \in \overline{\mathcal{M}}(n, r)$. We can view $T^{*} R$ as the representation space of dimension $(n, 1)$ for the double $\bar{Q}^{r}$ of the quiver $Q^{r}$ obtained from $Q$ by adjoining the additional vertex $\infty$ with $r$ arrows from the vertex 0 in $Q_{0}$ to $\infty$. Pick a semisimple representation of $\bar{Q}^{r}$ lying over $x$. This representation decomposes as $r^{0} \oplus r^{1} \otimes U_{1} \oplus \ldots \oplus r^{k} \otimes U_{k}$, where $r^{0}$ is an irreducible representation of $D Q^{w}$ with dimension $\left(n_{0}, 1\right)$ and $r^{1}, \ldots, r^{k}$ are pairwise nonisomorphic irreducible representations of $\bar{Q}$, all $r^{i}, i=1, \ldots, k$, have dimension 1 . Let $n_{i}=\operatorname{dim} U_{i}$.

According to [BL, 2.1.6], we have a decomposition $\overline{\mathcal{M}}(n, r)^{\wedge_{x}} \cong D \times \prod_{i=1}^{k} \overline{\mathcal{M}}\left(n_{i}, r\right)^{\wedge_{0}}$ of Poisson formal schemes, where $D$ stands for the symplectic formal disk and $\bullet{ }^{\wedge}$ indicates the formal neighborhood of $x$. From Lemma 3.6 it now follows that the locus described in the previous is a union of leaves. So in order to prove the entire proposition, it remains to show that the locus is irreducible. This easily follows from [CB, Theorem 1.2].

An isomorphism $\mathcal{M}(n, r)^{\wedge_{x}} \cong D \times \prod_{i=1}^{k} \bar{M}\left(n_{i}, r\right)^{\wedge_{0}}$ can be quantized (see [BL, Section $5.4])$ to

$$
\overline{\mathcal{A}}_{\lambda}(n, r)_{\hbar}^{\wedge_{0}}=\mathbf{A}_{\hbar}^{\wedge_{0}} \widehat{\otimes}_{\mathbb{C}[[\hbar]]} \overline{\mathcal{A}}_{\lambda}\left(n_{1}, r\right)_{\hbar}^{\wedge_{0}} \widehat{\otimes}_{\mathbb{C}[[\hbar]]} \cdots \widehat{\otimes}_{\mathbb{C}[\hbar]]]} \overline{\mathcal{A}}_{\lambda}\left(n_{k}, r\right)_{\hbar}^{\wedge_{0}}
$$

Here the notation is as follows. By $\mathbf{A}_{\hbar}^{\wedge_{0}}$ we mean the formal Weyl algebra quantizing $D$. The subscript $\hbar$ means the Rees algebra (with respect to the filtration by order of differential operators). We note that the map $\hat{r}$ from [BL, Section 5.4] sends $\lambda$ to $(\lambda, \lambda, \ldots, \lambda)$ hence all parameters in the right hand side of (3.2) are all equal to $\lambda$.

3.4. Harish-Chandra bimodules and restriction functors. Let $\mathcal{A}, \mathcal{A}^{\prime}$ be two quantizations of the same positively graded Poisson algebra $A$, where, for simplicity, the degree of the Poisson bracket is -1 . For example, we can take $A=\mathbb{C}[\overline{\mathcal{M}}(n, r)], \mathcal{A}=\overline{\mathcal{A}}_{\lambda}(n, r), \mathcal{A}^{\prime}=$ $\overline{\mathcal{A}}_{\lambda^{\prime}}(n, r)$ (with filtrations coming from the filtration on $D(\bar{R})$ by the order of differential operator). 
Let us recall the definition of a $\mathrm{HC} \mathcal{A}$ - $\mathcal{A}^{\prime}$-bimodule. By definition, this is $\mathcal{A}$ - $\mathcal{A}^{\prime}$-bimodule $\mathcal{B}$ that can be equipped with a good filtration, i.e., an $\mathcal{A}$ - $\mathcal{A}^{\prime}$-bimodule filtration subject to the following two properties:

- the induced left and right $A$-actions on gr $\mathcal{B}$ coincide,

- $\operatorname{gr} \mathcal{B}$ is a finitely generated $A$-module.

By a homomorphism of Harish-Chandra bimodules we mean a bimodule homomorphism. The category of $\mathrm{HC} \mathcal{A}$ - $\mathcal{A}^{\prime}$-bimodules is denoted by $\operatorname{HC}\left(\mathcal{A}-\mathcal{A}^{\prime}\right)$. We also consider the full subcategory $D_{H C}^{b}\left(\mathcal{A}-\mathcal{A}^{\prime}\right)$ of the derived category of $\mathcal{A}$ - $\mathcal{A}^{\prime}$-bimodules with HarishChandra homology.

By the associated variety of a $\mathrm{HC}$ bimodule $\mathcal{B}$ (denoted by $\mathrm{V}(\mathcal{B})$ ) we mean the support in $\operatorname{Spec}(A)$ of the coherent sheaf gr $\mathcal{B}$, where the associated graded is taken with respect to a good filtration. It is easy to see that gr $\mathcal{B}$ is a Poisson $A$-module so $\mathrm{V}(\mathcal{B})$ is the union of symplectic leaves (assuming $\operatorname{Spec}(A)$ has finitely many leaves).

Let $\mathcal{A}^{\prime \prime}$ be another filtered quantization of $A$. For $\mathcal{B}^{1} \in \mathrm{HC}\left(\mathcal{A}^{\prime}-\mathcal{A}\right)$ and $\mathcal{B}^{2} \in \mathrm{HC}\left(\mathcal{A}^{\prime \prime}-\mathcal{A}^{\prime}\right)$ we can take their tensor product $\mathcal{B}^{2} \otimes_{\mathcal{A}^{\prime}} \mathcal{B}^{1}$. This is easily seen to be a $\mathrm{HC} \mathcal{A}^{\prime \prime}-\mathcal{A}-$ bimodule. Also the derived tensor product of the objects from $D_{H C}^{b}\left(\mathcal{A}^{\prime \prime}-\mathcal{A}^{\prime}\right), D_{H C}^{b}\left(\mathcal{A}^{\prime}-\mathcal{A}\right)$ lies in $D_{H C}^{-}\left(\mathcal{A}^{\prime \prime}-\mathcal{A}\right)$ (and in $D_{H C}^{b}\left(\mathcal{A}^{\prime \prime}-\mathcal{A}\right)$ provided $\mathcal{A}^{\prime}$ has finite homological dimension).

We will need the following result from [L6] that is a part of [L6, Theorem 1.3].

Lemma 3.8. Let $\mathcal{A}$ be the algebra of global section of a quantization of a symplectic resolution. Then $\mathcal{A}$ has finite length as an $\mathcal{A}$ - $\mathcal{A}$-bimodule.

Now let us proceed to restriction functors defined in [BL, Section 5.4] for HC bimodules over the algebras $\overline{\mathcal{A}}_{\lambda}(n, r)$. Let us write $\overline{\mathcal{A}}_{\lambda}(n)$ for $\overline{\mathcal{A}}_{\lambda}(n, r)$. Pick a point $x$ in the leaf corresponding to the unordered collection $\mu=\left(n_{1}, \ldots, n_{k}\right)$ and set $\overline{\mathcal{A}}_{\lambda}(\mu)=\bigotimes_{i=1}^{k} \overline{\mathcal{A}}_{\lambda}\left(n_{i}\right)$. Then we have a functor

$$
\bullet_{\dagger, \mu}: \operatorname{HC}\left(\overline{\mathcal{A}}_{\lambda}(n)-\overline{\mathcal{A}}_{\lambda^{\prime}}(n)\right) \rightarrow \mathrm{HC}\left(\overline{\mathcal{A}}_{\lambda}(\mu)-\overline{\mathcal{A}}_{\lambda^{\prime}}(\mu)\right) \text {. }
$$

We will need several facts about the restriction functors established in [BL, Section $5.5]$.

Proposition 3.9. The following is true.

(1) The functor $\bullet_{\dagger, x}$ is exact and intertwines tensor products (as well as Tor's). It also intertwines one-sided Hom's (as well as Ext's).

(2) The associated variety $\mathrm{V}\left(\mathcal{B}_{\dagger, x}\right)$ is uniquely characterized by $D \times \mathrm{V}\left(\mathcal{B}_{\dagger, \mu}\right)^{\wedge_{0}}=\mathrm{V}(\mathcal{B})^{\wedge_{x}}$.

Now let $\mathcal{L}$ denote the leaf of $x$. Consider the subcategory $\operatorname{HC}_{\overline{\mathcal{L}}}\left(\overline{\mathcal{A}}_{\lambda}(n)-\overline{\mathcal{A}}_{\lambda^{\prime}}(n)\right)$ of all objects whose associated variety is contained in $\overline{\mathcal{L}}$. Now suppose that $\mathcal{L}$ corresponds to $\mu$. It follows that $\bullet_{\dagger, x}$ maps $\operatorname{HC}_{\overline{\mathcal{L}}}\left(\overline{\mathcal{A}}_{\lambda}(n)-\overline{\mathcal{A}}_{\lambda^{\prime}}(n)\right)$ to the category $\operatorname{HC}_{f i n}\left(\overline{\mathcal{A}}_{\lambda}(\mu)-\overline{\mathcal{A}}_{\lambda^{\prime}}(\mu)\right)$ of finite dimensional $\overline{\mathcal{A}}_{\lambda}(\mu)-\overline{\mathcal{A}}_{\lambda^{\prime}}(\mu)$-bimodules. As was shown in [L7, Section 3.3], this functor admits a right adjoint

$$
\bullet^{\dagger, x}: \mathrm{HC}_{f i n}\left(\overline{\mathcal{A}}_{\lambda}(\mu)-\overline{\mathcal{A}}_{\lambda^{\prime}}(\mu)\right) \rightarrow \mathrm{HC}_{\overline{\mathcal{L}}}\left(\overline{\mathcal{A}}_{\lambda}(n)-\overline{\mathcal{A}}_{\lambda^{\prime}}(n)\right) \text {. }
$$

We would like to point out that the results and constructions explained above in this section generalize to products of the algebras $\overline{\mathcal{A}}_{\lambda}(?, r)$ in a straightforward way.

Let us finish this section with a further discussion of wall-crossing bimodules. Namely, for $\lambda \in \mathbb{C}, \chi \in \mathbb{Z}$, we have the $\overline{\mathcal{A}}_{\lambda+\chi}(n, r)-\overline{\mathcal{A}}_{\lambda}(n, r)$-bimodule $\overline{\mathcal{A}}_{\lambda, \chi}^{0}(n, r):=\left[D(\bar{R}) / D(\bar{R})\left\{\xi_{R}-\right.\right.$ $\lambda \operatorname{tr} \xi \mid \xi \in \mathfrak{g}\}]^{G, \chi}$. This gives an example of a $\mathrm{HC} \overline{\mathcal{A}}_{\lambda+\chi}(n, r)-\overline{\mathcal{A}}_{\lambda}(n, r)$-bimodule.

Lemma 3.10. The following is true. 
(1) If $(\lambda+\chi, \theta)$ satisfies abelian localization, then $\overline{\mathcal{A}}_{\lambda, \chi}^{0}(n, r) \cong \overline{\mathcal{A}}_{\lambda, \chi}^{(\theta)}(n, r)$.

(2) $(\lambda, \theta)$ satisfies abelian localization if and only if, for all sufficiently large $n \in \mathbb{Z}$, the bimodules $\overline{\mathcal{A}}_{\lambda+m n \theta, n \theta}^{0}(n, r)$ and $\overline{\mathcal{A}}_{\lambda+(m+1) n \theta,-n \theta}^{0}(n, r)$ are mutually inverse Morita equivalences.

(3) If $x \in \overline{\mathcal{M}}(n, r)$ corresponds to a collection $\mu=\left(n_{1}, \ldots, n_{k}\right)$, then $\overline{\mathcal{A}}_{\lambda, \chi}^{0}(n, r)_{\dagger, x}=$ $\bigotimes_{i=1}^{k} \overline{\mathcal{A}}_{\lambda, \chi}^{0}\left(n_{i}, r\right)$

Proof. (1) is [BL, Lemma 5.25]. (2) follows from [BL, Lemma 5.26] and [BPW, Proposition 5.20]. (3) is a special case of [BL, (5.11)].

4. Finite dimensional RePresentations And Structure of CATEGory $\mathcal{O}$

4.1. Finite dimensional representations. The goal of this section is to prove the following statement.

Proposition 4.1. The sheaf $\overline{\mathcal{A}}_{\lambda}^{\theta}(n, r)$ has a coherent module supported on $\bar{\rho}^{-1}(0)$ if and only if $\lambda=\frac{s}{n}$ with s and $n$ coprime. If that is the case, then the category $\overline{\mathcal{A}}_{\lambda}^{\theta}(n, r)-\bmod _{\rho^{-1}(0)}$ of all coherent $\overline{\mathcal{A}}_{\lambda}^{\theta}(n, r)$-modules supported on $\rho^{-1}(0)$ is equivalent to Vect.

In the proof (and also below) we will need the following lemma.

Lemma 4.2. Let $\mathcal{C}$ be a highest weight category, where the classes of standard and costandard objects coincide. Let $R: D^{b}(\mathcal{C}) \stackrel{\sim}{\rightarrow} D^{b}\left(\mathcal{C}^{\vee}\right)$ be a Ringel duality functor. The following conditions are equivalent:

(1) $\mathcal{C}$ is semisimple.

(2) We have $H_{0}(R(L)) \neq 0$ for every simple object $L$.

(3) every simple lies in the head of a costandard object.

Proof. Recall that $R$ can be realized as $R \operatorname{Hom}_{\mathcal{C}}(T, \bullet)$, where $T$ stands for the sum of all indecomposable tiltings in $\mathcal{C}$. The implication $(1) \Rightarrow(2)$ is clear. The implication $(2) \Rightarrow(3)$ follows from the fact that every costandard object in a highest weight category is a quotient of a tilting.

Let us prove $(3) \Rightarrow(1)$. Let $\tau$ be a maximal (with respect to the coarsest highest weight ordering) label. Then the simple $L(\tau)$ lies in the head of some costandard, say $\nabla(\sigma)$. But all simple constituents of $\nabla(\sigma)$ are $L(\xi)$ with $\xi \leqslant \sigma$. It follows that $\sigma=\tau$. Since $L(\tau)$ lies in the head of $\nabla(\tau)$ and also coincides with the socle, we see that $\nabla(\tau)=L(\tau)$. So $L(\tau)$ is injective. Since the classes of standards and costandard coincide, it is also a projective object. So $L(\tau)$ itself forms a block.

Since this holds for any maximal $\tau$, we deduce that the category $\mathcal{C}$ is semisimple.

Proof of Proposition 4.1. The proof is in several steps.

Step 1. Let us take dominant $\nu$, i.e., $\nu(t)=(\alpha(t), t)$, where $\alpha(t)=\operatorname{diag}\left(t^{d_{1}}, \ldots, t^{d_{r}}\right)$ with $d_{1} \gg d_{2} \gg \ldots \gg d_{r}$. We can assume that $\lambda$ is as in Proposition 2.2. Let us show that for the category $\mathcal{O}\left(\overline{\mathcal{A}}_{\lambda}(n, r)\right)$ condition (2) of Lemma 4.2 is equivalent to all simples have support of dimension $r n-1$. By Lemmas 2.7 and 2.8, the functor $R$ is a perverse equivalence with respect to the filtration by dimension of support. So the claims that all simples have dimension of support equal to $r n-1$ is equivalent to $H_{0}(R L) \neq 0$ for all simple $L$. By Lemma 2.4, the classes of standards and costandards in $K_{0}\left(\mathcal{O}_{\nu}\left(\mathcal{A}_{\lambda}^{\theta}\right)\right)$ coincide and we are done by Lemma 4.2 .

Step 2. Suppose that $\lambda$ is Zariski generic and $\overline{\mathcal{A}}_{\lambda}(n, r)$ has a finite dimensional representation. Let us prove that the denominator of $\lambda$ is precisely $n$. Indeed, a finite 
dimensional module lies in the category $\mathcal{O}_{\nu}$. It follows that the algebra $C_{\nu_{0}}\left(\overline{\mathcal{A}}_{\lambda}(n, r)\right)$ has a finite dimensional representation, equivalently, there is a simple of GK dimension 1 in $\mathcal{O}_{\nu}\left(\mathrm{C}_{\nu_{0}}\left(\mathcal{A}_{\lambda}(n, r)\right)\right)$. Recall that $\mathrm{C}_{\nu_{0}}\left(\mathcal{A}_{\lambda}(n, r)\right)=\bigoplus \bigotimes_{i=1}^{r} \mathcal{A}_{\lambda}\left(n_{i}, 1\right)$, where the sum is taken over all $r$-multipartitions $\left(n_{1}, \ldots, n_{r}\right)$ of $n$. The minimal GK dimension of a module in the category $\mathcal{O}$ over $\bigotimes_{i=1}^{r} \mathcal{A}_{\lambda}\left(n_{i}, 1\right)$ is bounded from below by the number of positive integers among $n_{1}, \ldots, n_{r}$. So if there is a finite dimensional $\overline{\mathcal{A}}_{\lambda}(n, r)$-module, then there is a GK dimension 1 module in the category $\mathcal{O}_{\nu}\left(\mathcal{A}_{\lambda}(n, 1)\right)$, equivalently, a finite dimensional $\overline{\mathcal{A}}_{\lambda}(n, 1)$-module. The latter is a spherical rational Cherednik algebra for $S_{n}$. As was mentioned in Section 1.2, it has a finite dimensional representation only when the denominator of $\lambda$ is $n$.

Step 3. Let us prove that the algebra $\overline{\mathcal{A}}_{\lambda}(n, r)$ has no proper ideals of infinite codimension when $\lambda \notin \mathbb{Q}$ or the denominator of $\lambda$ is bigger than or equal to $n$. Indeed, let $\mathcal{I}$ be a proper ideal. Pick $x$ in an open symplectic leaf in $\mathrm{V}\left(\overline{\mathcal{A}}_{\lambda}(n, r) / \mathcal{I}\right)$ and consider the ideal $\mathcal{I}_{\dagger, x}$ in the slice algebra for $x$. The latter ideal has finite codimension by (2) of Proposition 3.9. By Section 3.3, this slice algebra is $\bigotimes_{i=1}^{k} \overline{\mathcal{A}}_{\lambda}\left(n_{i}, r\right)$, where $n_{1}, \ldots, n_{k}$ are positive integers with $\sum n_{i} \leqslant n$. So, by our assumption on $\lambda$ and Step 2, the slice algebra has no finite dimensional representations. We arrive at a contradiction, which proves the statement in the beginning of the step. In particular, we see that the algebra $\overline{\mathcal{A}}_{\lambda}(n, r)$ is simple when $\lambda$ is irrational or the denominator of $\lambda$ is bigger than $n$. We also see that in this case the category $\mathcal{O}$ is semisimple, this follows from Step 1 and Lemma 2.3.

Step 4. Now consider the case when $\lambda$ has denominator $n$. We claim that there is a finite dimensional module in $\mathcal{O}_{\nu}\left(\overline{\mathcal{A}}_{\lambda}(n, r)\right)$. The case when $n=1$ is easy, here $\overline{\mathcal{A}}_{\lambda}(1, r)=$ $D^{\lambda}\left(\mathbb{P}^{r-1}\right)$. So we assume that $n>1$.

By Lemma 2.3, for any $M \in \mathcal{O}_{\nu}\left(\overline{\mathcal{A}}_{\lambda}(n, r)\right)$, we have GK- $\operatorname{dim} M=\frac{1}{2} \operatorname{dim} \mathrm{V}\left(\overline{\mathcal{A}}_{\lambda}(n, r) / \operatorname{Ann} M\right)$. Now Step 3 implies that all simples in $\mathcal{O}_{\nu}$ are either finite dimensional or have support of dimension $r n-1$. If there are no finite dimensional modules, then thanks to Step 1, $\mathcal{O}\left(\overline{\mathcal{A}}_{\lambda}(n, r)\right)$ is semisimple.

Consider a one-parameter subgroups $\nu_{0}: t \mapsto(\alpha(t), 1)$, it is in a face of the chamber of $\nu$. The category $\mathcal{O}_{\nu}\left(\mathrm{C}_{\nu_{0}}\left(\mathcal{A}_{\lambda}(n, r)\right)\right)$ is not semisimple, it has the category $\mathcal{O}$ for the Rational Cherednik algebra with parameter $\lambda$ as a summand. By Proposition 2.5, $\mathcal{O}_{\nu}\left(\mathcal{A}_{\lambda}(n, r)\right)$ is not semisimple. Consequently, $\overline{\mathcal{A}}_{\lambda}(n, r)$ has a finite dimensional representation.

Step 5. Let us show that the number of irreducible coherent $\overline{\mathcal{A}}_{\lambda}^{\theta}(n, r)$-modules supported on $\rho^{-1}(0)$ cannot be bigger than 1 . Recall that to a module in the category $\mathcal{O}_{\nu}$ we can assign its characteristic cycle that is a formal linear combination of the irreducible components of the contracting locus of $\nu$. This map is injective, see BLPW, Section 6]. So it is enough to show that there is only one lagrangian irreducible component in $\rho^{-1}(0)$. Note that the lagrangian irreducible components of $\rho^{-1}(0)$ give a basis in $H^{2 n r-2}\left(\mathcal{M}^{\theta}(n, r)\right)$. According to [NY, Theorem 3.8], we have

$$
\sum_{i} \operatorname{dim} H^{2 i}\left(\mathcal{M}^{\theta}(n, r)\right) t^{i}=\sum_{\lambda} t^{\sum_{i=1}^{r}\left(r\left|\lambda^{(i)}\right|-i\left(\lambda^{(i) t}\right)_{1}\right)},
$$

where the summation is over the set of the $r$-multipartitions $\lambda=\left(\lambda^{(1)}, \ldots, \lambda^{(r)}\right)$. The highest power of $t$ in the right hand side is $r n-1$, it occurs for a single $\lambda$, namely, for $\lambda=((n), \varnothing, \ldots, \varnothing)$. This shows $\operatorname{dim} H^{2 n r-2}\left(\mathcal{M}^{\theta}(n, r)\right)=1$ and completes the proof of the claim in the beginning of this step.

Step 6. The previous step completes the proof of all claims of the theorem but the claim that the category of modules supported on $\rho^{-1}(0)$ is semisimple. The latter is an easy 
consequence of the observation that, in a highest weight category, we have $\operatorname{Ext}^{1}(L, L)=0$, for every simple $L$.

We would like to point out that the argument of Step 4 generalizes to the denominators less than $n$. So in those cases there are also simple $\overline{\mathcal{A}}_{\lambda}(n, r)$-modules of support with dimension $<r n-1$.

4.2. Proof of Theorem 1.4. In this subsection we will prove Theorem 1.4. We have already seen (Step 3 of the proof of Proposition 4.1) that if the denominator is bigger than $n$, then the category $\mathcal{O}$ is semisimple. The case of denominator $n$ will follow from a more precise statement, Theorem 4.3.

Let us introduce a certain model category. Let $\mathcal{C}_{n}$ denote the nontrivial block for the category $\mathcal{O}$ for the Rational Cherednik algebra $H_{1 / n}(n)$ for the symmetric group $\mathfrak{S}_{n}$. This is also the category of $B$-equivariant perverse sheaves on $\mathbb{P}^{n-1}$, where $B$ is a Borel subgroup of $\mathrm{PGL}_{n}$. Let us summarize some properties of this category.

(i) Its coarsest highest weight poset is linearly ordered: $p_{n}<p_{n-1}<\ldots<p_{1}$.

(ii) The projective objects $P\left(p_{i}\right)$ for $i>1$ are universal extensions $0 \rightarrow \Delta\left(p_{i-1}\right) \rightarrow$ $P\left(p_{i}\right) \rightarrow \Delta\left(p_{i}\right) \rightarrow 0$.

(iii) The indecomposable tilting objects $T\left(p_{i-1}\right)$ (with $\Delta\left(p_{i-1}\right)$ in the socle) for $i>1$ coincide with $P\left(p_{i}\right)$.

(iv) The simple objects $L\left(p_{i}\right)$ with $i>1$ appear in the heads of tiltings, while RHom $_{\mathcal{C}_{n}}\left(T, L\left(p_{1}\right)\right)$ is concentrated in homological degree $n$.

(v) There is a unique simple in the Ringel dual category $\mathcal{C}_{n}^{\vee}$ that appears in the higher cohomology of $\operatorname{RHom}_{\mathcal{C}_{n}}(T, \bullet)$.

Theorem 4.3. Consider a parameter of the form $\lambda=\frac{q}{n}$ with coprime $q, n$. Then the following is true.

(1) The category $\mathcal{O}_{\nu}\left(\overline{\mathcal{A}}_{\lambda}^{\theta}(n, r)\right)$ has only one nontrivial block that is equivalent to $\mathcal{C}_{r n}$. This block contains an irreducible representation supported on $\bar{\rho}^{-1}(0)$.

(2) Suppose that $\nu$ is dominant. Then the labels in the non-trivial block of $\mathcal{O}\left(\overline{\mathcal{A}}_{\lambda}^{\theta}(n, r)\right)$ are hooks $h_{i, d}=\left(\varnothing, \ldots,\left(n+1-d, 1^{d-1}\right), \ldots, \varnothing\right)$ (where $i$ is the number of the diagram where the hook appears) ordered by $h_{1, n}>h_{1, n-1}>\ldots>h_{1,1}>h_{2, n}>$ $\ldots>h_{2,1}>\ldots>h_{r, 1}$.

Proof. The proof is in several steps. We again deal with the realization of our category as $\mathcal{O}\left(\overline{\mathcal{A}}_{\lambda}(n, r)\right)$, where $\lambda$ is as in Proposition 2.2.

Step 1. As we have seen in the proof of Proposition 4.1, all simples have maximal dimension of support, except one, let us denote it by $L$, which is finite dimensional. So all blocks but one consist of modules with support of dimension $r n-1$. So (2) of Lemma 4.2 holds for these blocks and they are equivalent to Vect. Let $\mathcal{C}$ denote the block of $L$. The label of $L$, denote it by $p_{\max }$, is the largest in any highest weight ordering, this follows from the proof of $(3) \Rightarrow(1)$ of Lemma 4.2. For all other labels $p$ the simple $L(p)$ lies in the socle of the tilting generator $T$. In other words an analog of (iv) above holds for $\mathcal{C}$ with $r n$ instead of $n$. In the subsequent steps we will show that $\mathcal{C} \cong \mathcal{C}_{r n}$.

Step 2. Let us show that an analog of $(\mathrm{v})$ holds for $\mathcal{C}$. By Lemma 2.7, the higher homology of $\mathfrak{W C}_{\theta \rightarrow-\theta} L$ cannot have support of maximal dimension. It follows that the higher homology is finite dimensional and so is the direct sums of a single simple in $\mathcal{O}\left(\overline{\mathcal{A}}_{\lambda^{-}}(n, r)\right)$. Since $\mathfrak{W C}_{\theta \rightarrow-\theta}$ is a Ringel duality functor (Lemma 2.8), (v) follows. 
Step 3. Let us show that, in the coarsest highest weight order, there is a unique minimal label for $\mathcal{C}$, say $p_{\text {min }}$. This is equivalent to $\mathcal{C}^{\vee}$ having a unique maximal label because the orders on $\mathcal{C}$ and $\mathcal{C}^{\vee}$ are opposite. But $\mathcal{C}^{\vee}$ is equivalent to the nontrivial block in $\mathcal{O}\left(\overline{\mathcal{A}}_{-r-\lambda}(n, r)\right)$. So we are done by Step 1 (applied to $\left(\lambda^{-},-\theta\right)$ instead of $(\lambda, \theta)$ ) of this proof.

Step 4. Let us show that (v) implies that any tilting in $\mathcal{C}$ but one is projective. Let $R^{\vee}$ denote the Ringel duality equivalence $D^{b}\left(\mathcal{C}^{\vee}\right) \rightarrow D^{b}(\mathcal{C})^{o p p}$. We have $\operatorname{Ext}_{\mathcal{C}}^{i}\left(T(p), L\left(p^{\prime}\right)\right)=$ $\operatorname{Hom}_{\mathcal{C}}\left(T(p), L\left(p^{\prime}\right)[i]\right)=\operatorname{Hom}_{\mathcal{C}}\left(\left(R^{\vee}\right)^{-1} L\left(p^{\prime}\right)[i],\left(R^{\vee}\right)^{-1} T(p)\right)$. The objects $\left(R^{\vee}\right)^{-1} T(p)$ are injective so $\operatorname{Ext}^{i}\left(T(p), L\left(p^{\prime}\right)\right)=\operatorname{Hom}\left(H^{i}\left(\left(R^{\vee}\right)^{-1} L\left(p^{\prime}\right)\right),\left(R^{\vee}\right)^{-1} T(p)\right)$. Similarly to the previous step (applied to $\mathcal{C}^{\vee}$ instead of $\mathcal{C}$ and $\left(R^{\vee}\right)^{-1}$ instead of $R$ ), there is a unique indecomposable injective $I^{\vee}\left(p^{\vee}\right)$ in $\mathcal{C}^{\vee}$ that admits nonzero maps from a higher cohomology of $\left(R^{\vee}\right)^{-1} L(p)$. So if $\left(R^{\vee}\right)^{-1} T(p) \neq I^{\vee}\left(p^{\vee}\right)$, then $T(p)$ is projective.

Step 5. We remark that $\Delta\left(p_{\max }\right)$ is projective but not tilting, while $\Delta\left(p_{\min }\right)$ is tilting but not projective. So the projectives in $\mathcal{C}$ are $\Delta\left(p_{\max }\right)$ and $T(p)$ for $p \neq p_{\min }$. Similarly, the tiltings are $P(p), p \neq p_{\max }$, and $\Delta\left(p_{\min }\right)$.

Step 6. Let $\Lambda$ denote the highest weight poset for $\mathcal{C}$. Let us define a map $\nu: \Lambda \backslash\left\{p_{\text {min }}\right\} \rightarrow$ $\Lambda \backslash\left\{p_{\max }\right\}$. It follows from Step 5 that the head of any tilting in $\mathcal{C}$ is simple. By definition, $\nu(p)$ is such that $L(\nu(p))$ is the head of $T(p)$. We remark that $\nu(p)<p$ for any highest weight order, this follows from Step 4.

Step \%. Let us show that any element $p \in \Lambda$ has the form $\nu^{i}\left(p_{\max }\right)$. Assume the converse and let us pick the maximal element not of this form, say $p^{\prime}$. Since $p^{\prime} \neq p_{\max }$, we see that $L\left(p^{\prime}\right)$ lies in the head of some tilting. But the head of any indecomposable tilting is simple by Step 5 . So $\Delta\left(p^{\prime}\right)$ is a top term of a filtration with standard subsequent quotients. By the definition of $\nu$ and the choice of $p^{\prime}, \Delta\left(p^{\prime}\right)$ is tilting itself. Any indecomposable tilting but $\Delta\left(p_{\min }\right)$ is projective and we cannot have a standard that is projective and tilting simultaneously in a nontrivial block. So $p^{\prime}=p_{\text {min }}$. But let us pick a minimal element $p^{\prime \prime}$ in $\Lambda \backslash\left\{p_{\min }\right\}$. By Step 6, $\nu\left(p^{\prime \prime}\right)<p^{\prime \prime}$. So $\nu\left(p^{\prime \prime}\right)=p_{\text {min }}$. The claim in the beginning of the step is established. This proves (i) for $\mathcal{C}$.

Step 8. (ii) for $\mathcal{C}$ follows from Step 7 and (iii) follows from (ii) and Step 5.

Step 9. Let us show that $\# \Lambda=r n$. The minimal projective resolution for $\Delta\left(p_{\text {min }}\right)$ has length $\# \Lambda$, all projectives there are different, and the last term is $\Delta\left(p_{\max }\right)$. It follows that $\operatorname{RHom}\left(\Delta\left(p_{\min }\right), L\left(p_{\max }\right)\right)$ is concentrated in homological degree $\# \Lambda-1$. The other tiltings are projectives and RHom's with them amount to Hom's. Since RHom $\left(T, L\left(p_{\max }\right)\right)$ is concentrated in homological degree $r n-1$ (this follows from Lemmas 2.7] and 2.8), we are done.

Step 10. Let us complete the proof of (1). Let us order the labels in $\Lambda$ decreasingly, $p_{1}>\ldots>p_{r n}$. Using (ii) we get the following claims.

- $\operatorname{End}\left(P\left(p_{i}\right)\right)=\mathbb{C}[x] /\left(x^{2}\right)$ for $i>1$ and $\operatorname{End}\left(P\left(p_{1}\right)\right)=\mathbb{C}$.

- $\operatorname{Hom}\left(P\left(p_{i}\right), P\left(p_{j}\right)\right)$ is 1-dimensional if $|i-j|=1$ and is 0 if $|i-j|>1$.

Choose some basis elements $a_{i, i+1}, i=1, \ldots, r n-1$ in $\operatorname{Hom}\left(P\left(p_{i+1}\right), P\left(p_{i}\right)\right)$ and also basis elements $a_{i+1, i} \in \operatorname{Hom}\left(P\left(p_{i}\right), P\left(p_{i+1}\right)\right)$. We remark that the image of the composition $\operatorname{map} \operatorname{Hom}\left(P\left(p_{i+1}\right), P\left(p_{i}\right)\right) \times \operatorname{Hom}\left(P\left(p_{i}\right), P\left(p_{i+1}\right)\right) \rightarrow \operatorname{End}\left(P\left(p_{i}\right)\right)$ spans the maximal ideal. Choose generators $a_{i i}$ in the maximal ideals of $\operatorname{End}\left(P\left(p_{i}\right)\right), i=2, \ldots, r n$. Normalize $a_{21}$ by requiring that $a_{21} a_{12}=a_{22}$, automatically, $a_{12} a_{21}=0$. Normalize $a_{32}$ by $a_{23} a_{32}=a_{22}$ and then normalize $a_{33}$ by $a_{33}=a_{32} a_{23}$. We continue normalizing $a_{i+1, i}$ and $a_{i+1, i+1}$ in this way. We then recover the multiplication table in $\operatorname{End}\left(\bigoplus P\left(p_{i}\right)\right)$ in a unique way. This completes the proof of (1). 
Step 11. Now let us prove (2). Let us check that the labeling set $\Lambda$ for the nontrivial block of $\mathcal{O}\left(\overline{\mathcal{A}}_{\lambda}^{\theta}(n, r)\right)$ consists of hooks. For this, it is enough to check that $\Delta\left(h_{i, d}\right)$ does not form a block on itself. This is done similarly to Step 4 in the proof of Theorem 1.2. Now, according to [Ko], the hooks are ordered as specified in (2) with respect to the geometric order on the torus fixed points in $\mathcal{M}^{\theta}(n, r)$ (note that the sign conventions here and in [Ko] are different).

Remark 4.4. We can determine the label of the simple supported on $\bar{\rho}^{-1}(0)$ in the category $\mathcal{O}$ corresponding to an arbitrary generic torus. Namely, note that $\bar{\rho}^{-1}(0)$ coincides with the closure of a single contracting component and that contracting component corresponds to the maximal point. Now we can use results of [Ko] to find a label of the point: it always has only one nontrivial partition and this partition is either $(n)$ or $\left(1^{n}\right)$.

\section{LOCALizATION THEOREMS}

In this section we prove Theorem 1.1. The proof is in the following steps.

- We apply results of McGerty and Nevins, [MN2, to show that, first, if the abelian localization fails for $(\lambda, \theta)$, then $\lambda$ is a rational number with denominator not exceeding $n$, and, second, the parameters $\lambda=\frac{q}{m}$ with $m \leqslant n$ and $-r<\lambda<0$ are indeed singular and the functor $\Gamma_{\lambda}^{\theta}$ is exact when $\lambda>-r, \theta>0$ or $\lambda<0, \theta<0$. Thanks to an isomorphism $\mathcal{A}_{\lambda}^{\theta}(n, r) \cong \mathcal{A}_{-\lambda-r}^{-\theta}(n, r)$ (see Sections 2.1 and 3.1), this reduces the conjecture to checking that the abelian localization holds for $\lambda=\frac{q}{m}$ with $q \geqslant 0, m \leqslant n$ and $\theta>0$.

- Then we reduce the proof to the case when the denominator is precisely $n$ and $\lambda, \theta>0$.

- Then we will study a connection between the algebras $\mathrm{C}_{\nu_{0}}\left(\overline{\mathcal{A}}_{\lambda}(n, r)\right), \Gamma\left(\mathrm{C}_{\nu_{0}}\left(\overline{\mathcal{A}}_{\lambda}^{\theta}(n, r)\right)\right)$. We will show that the numbers of simples in the categories $\mathcal{O}$ for these algebras coincide. We deduce the localization theorem from there.

The last step is a crucial one and it does not generalize to other quiver varieties.

5.1. Results of McGerty and Nevins and consequences. In [MN2], McGerty and Nevins found a sufficient condition for the functor $\Gamma_{\lambda}^{\theta}: \mathcal{A}_{\lambda}^{\theta}(n, r)-\bmod \rightarrow \mathcal{A}_{\lambda}(n, r)$-mod to be exact (they were dealing with more general Hamiltonian reductions but we will only need the Gieseker case). Let us explain what their result gives in the case of interest for us. Consider the quotient functors $\pi_{\lambda}: D_{R}-\bmod ^{G, \lambda} \rightarrow \mathcal{A}_{\lambda}(n, r)$-mod and $\pi_{\lambda}^{\theta}: D_{R}-\bmod ^{G, \lambda} \rightarrow \mathcal{A}_{\lambda}^{\theta}(n, r)$-mod. Here $D_{R}$ stands for the sheaf of differential operators viewed as a microlocal sheaf on $T^{*} R$, and $D_{R}$-mod ${ }^{G, \lambda}$ is the category of $(G, \lambda)$-twisted equivariant D-modules on $R$. The functors $\pi_{\lambda}, \pi_{\lambda}^{\theta}$ are discussed in [BL, Section 2.3] or [BPW, Section 5.5].

Proposition 5.1. The inclusion $\operatorname{ker} \pi_{\lambda}^{\text {det }} \subset \operatorname{ker} \pi_{\lambda}$ holds provided $\lambda>-r$. Similarly, ker $\pi_{\lambda}^{\text {det }^{-1}} \subset \pi_{\lambda}$ provided $\lambda<0$.

I would like to thank Dmitry Korb for explaining me the required modifications to [MN2, Section 8].

Proof. We will consider the case $\theta=$ det, the opposite case follows from $\mathcal{A}_{\lambda}^{\theta}(n, r) \cong$ $\mathcal{A}_{-r-\lambda}^{-\theta}(n, r)$. The proof closely follows [MN2, Section 8], where the case of $r=1$ is considered. Instead of $R=\operatorname{End}(V) \oplus \operatorname{Hom}(V, W)$ they use $R^{\prime}=\operatorname{End}(V) \oplus \operatorname{Hom}(W, V)$, then, thanks to the partial Fourier transform, we have $D(R)-\bmod ^{G, \lambda} \cong D\left(R^{\prime}\right)-\bmod ^{G, \lambda+r}$. 
The set of weights in $R^{\prime}$ for a maximal torus $H \subset \mathrm{GL}(V)$ is independent of $r$ so we have the same Kempf-Ness subgroups as in the case $r=1$ : it is enough to consider the subgroups $\beta$ with tangent vectors (in the notation of [MN2, Section 8]) $e_{1}+\ldots+e_{k}$. The shift in loc.cit. becomes $\frac{r k}{2}$ (in the computation of loc.cit. we need to take the second summand $r$ times, that is all that changes). So we get that $\operatorname{ker} \pi_{\lambda}^{\text {det }} \subset \operatorname{ker} \pi_{\lambda}$ provided $k\left(-\frac{r}{2}-\lambda\right) \notin \frac{r k}{2}+\mathbb{Z}_{\geqslant 0}$ for all possible $k$ meaning $1 \leqslant k \leqslant n$ (the number $-\frac{r}{2}-\lambda$ is $c^{\prime}$ in loc.cit.). The condition simplifies to $\lambda \notin-r-\frac{1}{k} \mathbb{Z}_{\geqslant 0}$. This implies the claim of the proposition.

5.2. Reduction to denominator $n$ and singular parameters. Proposition 5.1 allows us to show that certain parameters are singular (meaning that the homological dimension of $\mathcal{A}_{\lambda}(n, r)$ is infinite).

Corollary 5.2. The parameters $\lambda$ with denominator $\leqslant n$ and $-r<\lambda<0$ are singular.

Proof. Assume the contrary. By Lemma 3.2, the functors $R \Gamma_{\lambda}^{ \pm \theta}$ are equivalences. Since the functors $\Gamma_{\lambda}^{ \pm \theta}$ are exact, we see that $\Gamma_{\lambda}^{ \pm \theta}$ are equivalences of abelian categories. From the inclusions $\operatorname{ker} \pi_{\lambda}^{ \pm \theta} \subset \operatorname{ker} \pi_{\lambda}$, we deduce that the functors $\pi_{\lambda}^{ \pm \theta}$ are isomorphic. So the wall-crossing functor $\mathfrak{W C}_{-\theta \leftarrow \theta}=\pi_{\lambda^{-}}^{-\theta} \circ\left(\mathbb{C}_{\lambda^{-}-\lambda} \otimes \bullet\right) \circ L \pi_{\lambda}^{\theta !}$ (see [BL, (2.10)] for the equality) is an equivalence of abelian categories (where we modify $\lambda$ by adding a sufficiently large integer). However, we have seen in the end of Section 4.1, the category $\mathcal{O}_{\nu}\left(\mathcal{A}_{\lambda}\right)$ is not semisimple. Combining Lemma 4.2 with Lemma 2.8, we see that $\mathfrak{W J C}_{-\theta \leftarrow \theta}$ cannot be an abelian equivalence.

Now let us observe that it is enough to check that the abelian localization holds for $\lambda \geqslant 0$ and $\theta>0$. This follows from an isomorphism $\mathcal{A}_{\lambda}^{\theta}(n, r) \cong \mathcal{A}_{-\lambda-r}^{-\theta}(n, r)$. This an isomorphism of sheaves on $\mathcal{M}^{\theta}(n, r) \cong \mathcal{M}^{-\theta}(n, r)$ (see the proof of Lemma 3.1).

Lemma 5.3. Suppose that, for all $n$, abelian localization holds for $\mathcal{A}_{\lambda}^{\theta}(n, r)$ if $\lambda>0$ and the denominator of $\lambda$ is exactly $n$. Then abelian localization holds for all $\lambda$.

Proof. Let the denominator $n^{\prime}$ of $\lambda$ be less then $n$. By Lemma 3.10, the abelian localization holds for $(\lambda, \theta>0)$ if and only if the bimodules $\mathcal{A}_{\lambda+m \chi, \chi}^{0}(n, r), \mathcal{A}_{\lambda+(m+1) \chi,-\chi}^{0}(n, r)$ with some $\chi \in \mathbb{Z}_{>0}$ define mutually dual Morita equivalences, equivalently, the natural homomorphisms

$$
\begin{aligned}
& \mathcal{A}_{\lambda+m \chi, \chi}^{0}(n, r) \otimes_{\mathcal{A}_{\lambda+m \chi}(n, r)} \mathcal{A}_{\lambda+(m+1) \chi,-\chi}^{0}(n, r) \rightarrow \mathcal{A}_{\lambda+(m+1) \chi}(n, r), \\
& \mathcal{A}_{\lambda+(m+1) \chi,-\chi}^{0}(n, r) \otimes_{\mathcal{A}_{\lambda+(m+1) \chi}(n, r)} \mathcal{A}_{\lambda+m \chi, \chi}^{0}(n, r) \rightarrow \mathcal{A}_{\lambda+m \chi}(n, r)
\end{aligned}
$$

are isomorphisms.

Assume the converse: there is $\lambda>0$ such that abelian localization does not hold for $\mathcal{A}_{\lambda}^{\theta}(n, r)$. Let $K^{1}, C^{1}, K^{2}, C^{2}$ denote the kernel and the cokernel of the first and of the second homomorphism (for some $m$ ), respectively. If one of these bimodules is nontrivial, then we can find $x \in \mathcal{M}(n, r)$ such that $K_{\dagger, x}^{i}, C_{\dagger, x}^{i}$ are finite dimensional and at least one of these bimodules is nonzero. From the classification of finite dimensional irreducibles, we see that the slice algebras must be of the form $\overline{\mathcal{A}}_{\lambda}\left(n^{\prime}, r\right)^{\otimes k}$. But, by Lemma 3.10 , $\mathcal{A}_{\lambda+(m+1) \chi,-\chi}^{0}(n, r)_{\dagger, x}=\overline{\mathcal{A}}_{\lambda+(m+1) \chi,-\chi}^{0}\left(n^{\prime}, r\right)^{\otimes k}, \mathcal{A}_{\lambda+m \chi, \chi}^{0}(n, r)_{\dagger, x}=\overline{\mathcal{A}}_{\lambda+m \chi, \chi}^{0}\left(n^{\prime}, r\right)^{\otimes k}$. Further, applying $\bullet_{\dagger, x}$ to (5.1) we again get natural homomorphisms. But the localization theorem holds for the algebra $\overline{\mathcal{A}}_{\lambda}\left(n^{\prime}, r\right)$ thanks to our inductive assumption, so the homomorphisms of the $\overline{\mathcal{A}}_{\lambda}\left(n^{\prime}, r\right)^{\otimes k}$-bimodules are isomorphisms. This contradiction finishes the proof of the lemma. 
5.3. Number of simples in $\mathcal{O}\left(\mathcal{A}_{\lambda}(n, r)\right)$. So we need to prove that the localization theorem holds for positive parameters $\lambda$ with denominator $n$ (the case $\lambda=0$ occurs only if $n=1$ and in that case this is a classical localization theorem for differential operators on projective spaces). We will derive the proof from the claim that the number of simple objects in the categories $\mathcal{O}_{\nu}\left(\overline{\mathcal{A}}_{\lambda}(n, r)\right)$ and $\mathcal{O}_{\nu}\left(\overline{\mathcal{A}}_{\lambda}^{\theta}(n, r)\right)$ is the same. For this we will need to study the natural homomorphism

$$
\varphi: \mathrm{C}_{\nu_{0}}\left(\overline{\mathcal{A}}_{\lambda}(n, r)\right) \rightarrow \Gamma\left(\mathrm{C}_{\nu_{0}}\left(\overline{\mathcal{A}}_{\lambda}^{\theta}(n, r)\right)\right) .
$$

Here, as before, $\nu(t)=(\alpha(t), t), \nu_{0}(t)=(\alpha(t), 1)$, where $\alpha: \mathbb{C}^{\times} \rightarrow \mathrm{GL}(r)$ is of the form $t \mapsto\left(t^{d_{1}}, \ldots, t^{d_{r}}\right)$, and $d_{1} \gg d_{2} \gg \ldots \gg d_{r}$.

Recall, see Proposition 3.5, that $\Gamma\left(C_{\nu_{0}}\left(\overline{\mathcal{A}}_{\lambda}^{\theta}(n, r)\right)\right)=\bigoplus \overline{\mathcal{A}}_{\lambda}\left(n_{1}, \ldots, n_{r} ; r\right)$, where the summation is taken over all compositions $n=n_{1}+\ldots+n_{r}$ and $\overline{\mathcal{A}}_{\lambda}\left(n_{1}, \ldots, n_{r} ; r\right) \otimes D(\mathbb{C})=$ $\bigotimes_{i=1}^{r} \mathcal{A}_{\lambda+i-1}\left(n_{i}, 1\right)$ (the factor $D(\mathbb{C})$ is embedded into the right hand side "diagonally"). Let $\mathcal{B}$ denote the maximal finite dimensional quotient of $\Gamma\left(\mathrm{C}_{\nu_{0}}\left(\overline{\mathcal{A}}_{\lambda}^{\theta}(n, r)\right)\right)$.

Proposition 5.4. The composition of $\varphi$ with the projection $\Gamma\left(\mathrm{C}_{\nu_{0}}\left(\overline{\mathcal{A}}_{\lambda}^{\theta}(n, r)\right)\right) \rightarrow \mathcal{B}$ is surjective.

Proof. The proof is in several steps.

Step 1. We claim that it is sufficient to prove that the composition $\varphi_{i}$ of $\varphi$ with the projection $\Gamma\left(\mathrm{C}_{\nu_{0}}\left(\overline{\mathcal{A}}_{\lambda}^{\theta}(n, r)\right)\right) \rightarrow \overline{\mathcal{A}}_{\lambda+i}(n, 1)$ is surjective. Indeed, each $\overline{\mathcal{A}}_{\lambda+i}(n, 1), i=$ $0, \ldots, r-1$ has a unique finite dimensional representation. The dimensions of these representations are pairwise different, see [BEG]. Namely, if $\lambda=\frac{q}{n}$, then the dimension is $\frac{(q+n-1) !}{q ! n !}$. So $\mathcal{B}$ is the sum of $r$ pairwise non-isomorphic matrix algebras. Therefore the surjectivity of the homomorphism $\mathrm{C}_{\nu_{0}}\left(\overline{\mathcal{A}}_{\lambda}(n, r)\right) \rightarrow \mathcal{B}$ follows from the surjectivity of all its $r$ components. We remark that the other summands of $\mathrm{C}_{\nu_{0}}\left(\overline{\mathcal{A}}_{\lambda}(n, r)\right)$ have no finite dimensional representations.

Step 2. Generators of $\overline{\mathcal{A}}_{\lambda+i}(n, 1)$ are known. Namely, recall that $\overline{\mathcal{A}}_{\lambda+i}(n, 1)$ is the spherical subalgebra in the Cherednik algebra $H_{c}(n)$ for the reflection representation $\mathfrak{h}$ of $\mathfrak{S}_{n}$ with $c=\lambda+i$. The algebra $H_{c}(n)$ is generated by $\mathfrak{h}, \mathfrak{h}^{*}$. Then the algebra $e H_{c}(n) e$ is generated by $S(\mathfrak{h})^{W}, S\left(\mathfrak{h}^{*}\right)^{W}$, see, e.g., the proof [EG, Proposition 4.9]. On the level of quantum Hamiltonian reduction, $S(\mathfrak{h})^{W}$ coincides with the image of $S(\overline{\mathfrak{g}})^{G} \subset D\left(\overline{\mathfrak{g}} \oplus \mathbb{C}^{n *}\right)^{G}$, while $S\left(\mathfrak{h}^{*}\right)^{W}$ coincides with the image of $S\left(\overline{\mathfrak{g}}^{*}\right)^{G}$. Here and below we write $\overline{\mathfrak{g}}$ for $\mathfrak{s l}_{n}$. We will show that the images of $S(\overline{\mathfrak{g}})^{G}, S\left(\overline{\mathfrak{g}}^{*}\right)^{G}$ lie in the image of $\varphi_{i}: \mathrm{C}_{\nu_{0}}\left(\overline{\mathcal{A}}_{\lambda}(n, r)\right) \rightarrow$ $\overline{\mathcal{A}}_{\lambda+i}(n, 1)$, this will establish the surjectivity in Step 1.

Step 3. Let us produce a natural homomorphism $S\left(\overline{\mathfrak{g}}^{*}\right)^{G} \rightarrow \mathrm{C}_{\nu_{0}}\left(\overline{\mathcal{A}}_{\lambda}(n, r)\right)$. First of all, recall that $\overline{\mathcal{A}}_{\lambda}(n, r)$ is a quotient of $D\left(\overline{\mathfrak{g}} \oplus\left(\mathbb{C}^{* n}\right)^{r}\right)^{G}$. The algebra $S\left(\overline{\mathfrak{g}}^{*}\right)^{G}$ is included into $D\left(\overline{\mathfrak{g}} \oplus\left(\mathbb{C}^{* n}\right)^{\oplus r}\right)^{G}$ as the algebra of invariant functions on $\overline{\mathfrak{g}}$. So we get a homomorphism $S\left(\overline{\mathfrak{g}}^{*}\right)^{G} \rightarrow \overline{\mathcal{A}}_{\lambda}(n, r)$. Since the $\mathbb{C}^{\times}$-action $\nu_{0}$ used to form $\mathrm{C}_{\nu_{0}}\left(\overline{\mathcal{A}}_{\lambda}(n, r)\right)$ is nontrivial only on $\left(\mathbb{C}^{* n}\right)^{\oplus r}$, we see that the image of $S\left(\overline{\mathfrak{g}}^{*}\right)^{G}$ lies in $\overline{\mathcal{A}}_{\lambda}(n, r)^{\nu_{0}\left(\mathbb{C}^{\times}\right)}$. So we get a homomorphism $\iota: S\left(\overline{\mathfrak{g}}^{*}\right)^{G} \rightarrow \mathrm{C}_{\nu_{0}}\left(\overline{\mathcal{A}}_{\lambda}(n, r)\right)$.

Step 4. We claim that $\varphi_{i} \circ \iota$ coincides with the inclusion $S\left(\overline{\mathfrak{g}}^{*}\right)^{G} \rightarrow \overline{\mathcal{A}}_{\lambda+i}(n, 1)$. We can filter the algebra $D\left(\overline{\mathfrak{g}} \oplus\left(\mathbb{C}^{* n}\right)^{\oplus r}\right)$ by the order of a differential operator. This induces filtrations on $\overline{\mathcal{A}}_{\lambda}(n, r), \overline{\mathcal{A}}_{\lambda}^{\theta}(n, r)$. We have similar filtrations on the algebras $\overline{\mathcal{A}}_{\lambda+i}(n, 1)$. The filtrations on $\overline{\mathcal{A}}_{\lambda}(n, r), \overline{\mathcal{A}}_{\lambda}^{\theta}(n, r)$ are preserved by $\nu_{0}$ and hence we have filtrations on $\mathrm{C}_{\nu_{0}}\left(\overline{\mathcal{A}}_{\lambda}(n, r)\right), \Gamma\left(\mathrm{C}_{\nu_{0}}\left(\overline{\mathcal{A}}_{\lambda}^{\theta}(n, r)\right)\right)$. It is clear from the construction of the projection $\Gamma\left(\mathrm{C}_{\nu_{0}}\left(\overline{\mathcal{A}}_{\lambda}^{\theta}(n, r)\right)\right) \rightarrow \overline{\mathcal{A}}_{\lambda+i}(n, 1)$ that it is compatible with the filtrations. On the other hand, the images of $S\left(\overline{\mathfrak{g}}^{*}\right)^{G}$ in both $\mathrm{C}_{\nu_{0}}\left(\overline{\mathcal{A}}_{\lambda}(n, r)\right), \overline{\mathcal{A}}_{\lambda+i}(n, 1)$ lies in the filtration degree 
0. So it is enough to prove the coincidence of the homomorphisms in the beginning of the step after passing to associate graded algebras.

Step 5. The associated graded homomorphisms coincide with analogous homomorphisms defined on the classical level. The components of $\mathcal{M}^{\theta}(n, r)^{\nu_{0}\left(\mathbb{C}^{\times}\right)}$that are Hilbert schemes are realized as follows. Pick an eigenbasis $w_{1}, \ldots, w_{r} \in \mathbb{C}^{r}$ for $T_{0}$. Then the Hilbert scheme component $Z_{i}$ of $\mathcal{M}^{\theta}(n, r)^{T_{0}}$ corresponding to the composition $\left(0^{i-1}, n, 0^{r-i-1}\right)$ consists of the $G$-orbits of $(A, B, 0, j)$, where $j: \mathbb{C}^{n} \rightarrow \mathbb{C}^{r}$ is a map with image in $\mathbb{C} w_{j}$. In particular, the homomorphism $S\left(\overline{\mathfrak{g}}^{*}\right)^{G} \rightarrow \operatorname{gr} \mathcal{A}_{\lambda+i}(n, 1)$ is dual to the morphism given by $(A, B, 0, j) \rightarrow A$.

On the other hand, $Z_{i}$ maps to $\mathcal{M}(r, n) / / \nu_{0}\left(\mathbb{C}^{\times}\right)\left(Z_{i} \hookrightarrow \mathcal{M}^{\theta}(n, r) \rightarrow \mathcal{M}(n, r) \rightarrow\right.$ $\left.\mathcal{M}(n, r) / / \nu_{0}\left(\mathbb{C}^{\times}\right)\right)$. The corresponding homomorphism of algebras is the associated graded of $\overline{\mathcal{A}}_{\lambda}(n, r)^{\nu_{0}\left(\mathbb{C}^{\times}\right)} \rightarrow \overline{\mathcal{A}}_{\lambda+i}(n, 1)$. Then we have the morphism $\mathcal{M}(r, n) / / \nu_{0}\left(\mathbb{C}^{\times}\right) \rightarrow \overline{\mathfrak{g}} / / G$ induced by $(A, B, 0, j) \mapsto A$. The corresponding homomorphism of algebras is the associated graded of $S\left(\overline{\mathfrak{g}}^{*}\right)^{G} \rightarrow \overline{\mathcal{A}}_{\lambda}(n, r)^{\nu_{0}\left(\mathbb{C}^{\times}\right)}$. So we have checked that the associated graded homomorphism of $\varphi_{i} \circ \iota: S\left(\overline{\mathfrak{g}}^{*}\right)^{G} \rightarrow \overline{\mathcal{A}}_{\lambda+i}(n, 1)$ coincides with that of the embedding $S\left(\overline{\mathfrak{g}}^{*}\right)^{G} \rightarrow \overline{\mathcal{A}}_{\lambda+i}(n, 1)$. This proves the claim of Step 4 .

Step 6. The coincidence of similar homomorphisms $S(\overline{\mathfrak{g}})^{G} \rightarrow \overline{\mathcal{A}}_{\lambda+i}(n, 1)$ is established analogously. The proof of the surjectivity of $\mathrm{C}_{\nu_{0}}\left(\overline{\mathcal{A}}_{\lambda}(n, r)\right) \rightarrow \overline{\mathcal{A}}_{\lambda+i}(n, 1)$ is now complete.

We still have a Hamiltonian action of $\mathbb{C}^{\times}$on $\mathrm{C}_{\nu_{0}}\left(\overline{\mathcal{A}}_{\lambda}(n, r)\right)$ (via $\nu$ ) that makes the homomorphism $\mathrm{C}_{\nu_{0}}\left(\overline{\mathcal{A}}_{\lambda}(n, r)\right) \rightarrow \Gamma\left(\mathrm{C}_{\nu_{0}}\left(\overline{\mathcal{A}}_{\lambda}^{\theta}(n, r)\right)\right)$ equivariant. So we can form the category $\mathcal{O}\left(\mathrm{C}_{\nu_{0}}\left(\overline{\mathcal{A}}_{\lambda}(n, r)\right)\right)$ for this action. By [L5, Section 5.5], we have an isomorphism $\mathrm{C}_{\nu}\left(\mathrm{C}_{\nu_{0}}\left(\overline{\mathcal{A}}_{\lambda}(n, r)\right)\right) \cong \mathrm{C}_{\nu}\left(\overline{\mathcal{A}}_{\lambda}(n, r)\right)$. So there is a natural bijection between the sets of simples in $\mathcal{O}_{\nu}\left(\mathrm{C}_{\nu_{0}}\left(\overline{\mathcal{A}}_{\lambda}(n, r)\right)\right)$ and in $\mathcal{O}_{\nu}\left(\overline{\mathcal{A}}_{\lambda}(n, r)\right)$.

Proposition 5.5. The number of simples in $\mathcal{O}\left(\mathrm{C}_{\nu_{0}}\left(\overline{\mathcal{A}}_{\lambda}(n, r)\right)\right)$ is bigger than or equal to that in $\mathcal{O}\left(\Gamma\left(\mathrm{C}_{\nu_{0}}\left(\overline{\mathcal{A}}_{\lambda}^{\theta}(n, r)\right)\right)\right)$.

Proof. The proof is again in several steps.

Step 1. We have a natural homomorphism $\mathbb{C}[\overline{\mathfrak{g}}]^{G} \rightarrow \bigoplus \overline{\mathcal{A}}_{\lambda}\left(n_{1}, \ldots, n_{r} ; r\right)$. It can be described as follows. We have an identification $\mathbb{C}[\overline{\mathfrak{g}}]^{G} \cong \mathbb{C}[\mathfrak{h}]^{\mathfrak{S}_{n}}$. This algebra embeds into $\overline{\mathcal{A}}_{\lambda}\left(n_{1}, \ldots, n_{r} ; r\right)$ (that is a spherical Cherednik algebra for the group $\prod_{i=1}^{r} \mathfrak{S}_{n_{i}}$ act-

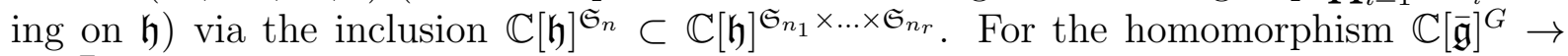
$\bigoplus \overline{\mathcal{A}}_{\lambda}\left(n_{1}, \ldots, n_{r} ; r\right)$ we take the direct sum of these embeddings. Similarly to Steps 4,5 of the proof of Proposition 5.4, the maps $\mathbb{C}[\overline{\mathfrak{g}}]^{G} \rightarrow \mathrm{C}_{\nu_{0}}\left(\overline{\mathcal{A}}_{\lambda}(n, r)\right), \Gamma\left(\mathrm{C}_{\nu_{0}}\left(\overline{\mathcal{A}}_{\lambda}^{\theta}(n, r)\right)\right)$ are intertwined by the homomorphism $\mathrm{C}_{\nu_{0}}\left(\overline{\mathcal{A}}_{\lambda}(n, r)\right) \rightarrow \Gamma\left(\mathrm{C}_{\nu_{0}}\left(\overline{\mathcal{A}}_{\lambda}^{\theta}(n, r)\right)\right)$.

Step 2. Let $\delta \in \mathbb{C}[\overline{\mathfrak{g}}]^{G}$ be the discriminant. We claim that $\mathrm{C}_{\nu_{0}}\left(\overline{\mathcal{A}}_{\lambda}^{\theta}(n, r)\right)\left[\delta^{-1}\right] \stackrel{\sim}{\rightarrow}$ $\Gamma\left(\mathrm{C}_{\nu_{0}}\left(\overline{\mathcal{A}}_{\lambda}^{\theta}(n, r)\right)\right)\left[\delta^{-1}\right]$. Since $\delta$ is $\nu_{0}\left(\mathbb{C}^{\times}\right)$-stable, we have $\mathrm{C}_{\nu_{0}}\left(\overline{\mathcal{A}}_{\lambda}(n, r)\right)\left[\delta^{-1}\right]=\mathrm{C}_{\nu_{0}}\left(\overline{\mathcal{A}}_{\lambda}(n, r)\left[\delta^{-1}\right]\right)$. We will describe the algebra $\mathrm{C}_{\nu_{0}}\left(\overline{\mathcal{A}}_{\lambda}(n, r)\left[\delta^{-1}\right]\right)$ explicitly and see that $\mathrm{C}_{\nu_{0}}\left(\overline{\mathcal{A}}_{\lambda}(n, r)\left[\delta^{-1}\right]\right) \stackrel{\sim}{\rightarrow}$ $\Gamma\left(\mathrm{C}_{\nu_{0}}\left(\overline{\mathcal{A}}_{\lambda}^{\theta}(n, r)\right)\right)\left[\delta^{-1}\right]$.

Step 3. We start with the description of $\overline{\mathcal{A}}_{\lambda}(n, r)\left[\delta^{-1}\right]$. Let $\overline{\mathfrak{g}}^{\text {reg }}$ denote the locus of the regular semisimple elements in $\overline{\mathfrak{g}}$. Then $\overline{\mathcal{A}}_{\lambda}(n, r)\left[\delta^{-1}\right]=D\left(\overline{\mathfrak{g}}^{\text {reg }} \times \operatorname{Hom}\left(\mathbb{C}^{n}, \mathbb{C}^{r}\right)\right) / / /{ }_{\lambda} G$. Here /// $\lambda_{\lambda}$ denotes the quantum Hamiltonian reduction with parameter $\lambda$. 
Recall that $\overline{\mathfrak{g}}^{\text {reg }}=G \times_{N_{G}(\mathfrak{h})} \mathfrak{h}^{\text {reg }}$ and so $\overline{\mathfrak{g}}^{\text {reg }} \times \operatorname{Hom}\left(\mathbb{C}^{n}, \mathbb{C}^{r}\right)=G \times_{N_{G}(\mathfrak{h})}\left(\mathfrak{h}^{\text {reg }} \times\right.$ $\left.\operatorname{Hom}\left(\mathbb{C}^{n}, \mathbb{C}^{r}\right)\right)$. It follows that

$$
\begin{aligned}
& D\left(\overline{\mathfrak{g}}^{\text {reg }} \times \operatorname{Hom}\left(\mathbb{C}^{n}, \mathbb{C}^{r}\right)\right) / / /{ }_{\lambda} G=D\left(\mathfrak{h}^{\text {reg }} \times \operatorname{Hom}\left(\mathbb{C}^{n}, \mathbb{C}^{r}\right)\right) / / /{ }_{\lambda} N_{G}(\mathfrak{h})= \\
& \left(D\left(\mathfrak{h}^{r e g}\right) \otimes D\left(\operatorname{Hom}\left(\mathbb{C}^{n}, \mathbb{C}^{r}\right)\right) / /{ }_{\lambda} H\right)^{\mathfrak{S}_{n}}=\left(D\left(\mathfrak{h}^{r e g}\right) \otimes D^{\lambda}\left(\mathbb{P}^{r-1}\right)^{\otimes n}\right)^{\mathfrak{S}_{n}} .
\end{aligned}
$$

Here, in the second line, we write $H$ for the Cartan subgroup of $G=\mathrm{GL}_{n}(\mathbb{C})$ and take the diagonal action of $\mathfrak{S}_{n}$. In the last expression, it permutes the tensor factors. A similar argument shows that $\overline{\mathcal{M}}^{\theta}(n, r)_{\delta}=\left(T^{*}\left(\mathfrak{h}^{r e g}\right) \times T^{*}\left(\mathbb{P}^{r-1}\right)^{n}\right) / \mathfrak{S}_{n}$ and the restriction of $\overline{\mathcal{A}}_{\lambda}^{\theta}(n, r)$ to this open subset is $\left(D_{\mathfrak{h}^{\text {reg }}} \otimes\left(D_{\mathbb{P}^{r-1}}^{\lambda}\right)^{\otimes n}\right)^{\mathfrak{S}_{n}}$.

Step 4. Now we are going to describe the algebra $C_{\nu_{0}}\left(\left(D\left(\mathfrak{h}^{\text {reg }}\right) \otimes D^{\lambda}\left(\mathbb{P}^{r-1}\right)^{\otimes n}\right)^{\mathfrak{S}_{n}}\right)$. First of all, we claim that

$$
\mathrm{C}_{\nu_{0}}\left(\left(D\left(\mathfrak{h}^{r e g}\right) \otimes D^{\lambda}\left(\mathbb{P}^{r-1}\right)^{\otimes n}\right)^{\mathfrak{S}_{n}}\right)=\left(\mathrm{C}_{\nu_{0}}\left(D\left(\mathfrak{h}^{r e g}\right) \otimes D^{\lambda}\left(\mathbb{P}^{r-1}\right)^{\otimes n}\right)\right)^{\mathfrak{S}_{n}}
$$

There is a natural homomorphism from the left hand side to the right hand side. To prove that it is an isomorphism one can argue as follows. First, note, that since the $\mathfrak{S}_{n}$-action on $\mathfrak{h}^{\text {reg }}$ is free, we have

$$
D\left(\mathfrak{h}^{r e g}\right) \otimes D^{\lambda}\left(\mathbb{P}^{r-1}\right)^{\otimes n}=D\left(\mathfrak{h}^{\text {reg }}\right) \otimes_{D\left(\mathfrak{h}^{\text {reg }}\right)^{\mathfrak{S}_{n}}}\left(D\left(\mathfrak{h}^{\text {reg }}\right) \otimes D^{\lambda}\left(\mathbb{P}^{r-1}\right)^{\otimes n}\right)^{\mathfrak{S}_{n}}
$$

Since $D\left(\mathfrak{h}^{\text {reg }}\right)$ is $\nu_{0}\left(\mathbb{C}^{\times}\right)$-invariant, the previous equality implies (5.2).

Step 5. Now let us describe $\mathrm{C}_{\nu_{0}}\left(\left(D\left(\mathfrak{h}^{r e g}\right) \otimes D^{\lambda}\left(\mathbb{P}^{r-1}\right)^{\otimes n}\right)=D\left(\mathfrak{h}^{r e g}\right) \otimes \mathcal{C}_{\nu_{0}}\left(\left(D^{\lambda}\left(\mathbb{P}^{r-1}\right)\right)^{\otimes n}\right)\right.$. The $\mathbb{C}^{\times}$-action on the tensor product $\left(D^{\lambda}\left(\mathbb{P}^{r-1}\right)\right)^{\otimes n}$ is diagonal and it is easy to see that $\mathrm{C}_{\nu_{0}}\left(\left(D^{\lambda}\left(\mathbb{P}^{r-1}\right)\right)^{\otimes n}\right)=\left(\mathrm{C}_{\nu_{0}}\left(D^{\lambda}\left(\mathbb{P}^{r-1}\right)\right)\right)^{\otimes n}$. So we need to compute $\mathrm{C}_{\nu_{0}}\left(D^{\lambda}\left(\mathbb{P}^{r-1}\right)\right)$. We claim that this algebra is isomorphic to $\mathbb{C}^{\oplus r}$. Indeed, $D^{\lambda}\left(\mathbb{P}^{r-1}\right)$ is a quotient of the central reduction $U_{\tilde{\lambda}}\left(\mathfrak{s l}_{r}\right)$ of $U\left(\mathfrak{s l}_{r}\right)$ at the central character $\tilde{\lambda}:=\lambda \omega_{r}$. We remark that $\lambda \omega_{r}+\rho$ is regular because $\lambda \geqslant 0$. We have $C_{\nu_{0}}\left(U_{\tilde{\lambda}}\left(\mathfrak{s l}_{r}\right)\right)=\mathbb{C}^{\oplus r !}$ and $\mathrm{C}_{\nu_{0}}\left(D^{\lambda}\left(\mathbb{P}^{r-1}\right)\right)$ is a quotient of that. The number of irreducible representations of $\mathrm{C}_{\nu_{0}}\left(D^{\lambda}\left(\mathbb{P}^{r-1}\right)\right)$ equals to the number of simples in the category $\mathcal{O}$ for $D^{\lambda}\left(\mathbb{P}^{r-1}\right)$ that coincides with $r$ since abelian localization holds. An isomorphism $\mathrm{C}_{\nu_{0}}\left(D^{\lambda}\left(\mathbb{P}^{r-1}\right)\right)=\mathbb{C}^{\oplus r}$ follows.

Step 6. So we see that $\mathrm{C}_{\nu_{0}}\left(\overline{\mathcal{A}}_{\lambda}(n, r)\left[\delta^{-1}\right]\right)=\left(D\left(\mathfrak{h}^{\text {reg }}\right) \otimes\left(\mathbb{C}^{\oplus r}\right)^{\otimes n}\right)^{\mathfrak{S}_{n}}$. By similar reasons, we have $\Gamma\left(\left[\overline{\mathcal{M}}^{\theta}(n, r)_{\delta}\right]^{\nu_{0}\left(\mathbb{C}^{\times}\right)}, \mathrm{C}_{\nu_{0}}\left(\overline{\mathcal{A}}_{\lambda}^{\theta}(n, r)\right)\right)=\left(D\left(\mathfrak{h}^{r e g}\right) \otimes\left(\mathbb{C}^{\oplus r}\right)^{\otimes n}\right)^{\mathfrak{S}_{n}}$. The natural homomorphism

$$
\mathrm{C}_{\nu_{0}}\left(\overline{\mathcal{A}}_{\lambda}(n, r)\left[\delta^{-1}\right]\right) \rightarrow \Gamma\left(\left(\overline{\mathcal{M}}^{\theta}(n, r)_{\delta}\right)^{\nu_{0}\left(\mathbb{C}^{\times}\right)}, \mathrm{C}_{\nu_{0}}\left(\overline{\mathcal{A}}_{\lambda}^{\theta}(n, r)\right)\right)
$$

is an isomorphism by the previous two steps. Also we have a natural homomorphism

$$
\Gamma\left(\mathrm{C}_{\nu_{0}}\left(\overline{\mathcal{A}}_{\lambda}^{\theta}(n, r)\right)\right)\left[\delta^{-1}\right] \rightarrow \Gamma\left(\left[\mathcal{M}^{\theta}(n, r)_{\delta}\right]^{\nu_{0}\left(\mathbb{C}^{\times}\right)}, \mathrm{C}_{\nu_{0}}\left(\overline{\mathcal{A}}_{\lambda}^{\theta}(n, r)\right)\right) .
$$

The latter homomorphism is an isomorphism from the explicit description of $\mathrm{C}_{\nu_{0}}\left(\overline{\mathcal{A}}_{\lambda}^{\theta}(n, r)\right)$. Indeed, $\mathrm{C}_{\nu_{0}}\left(\overline{\mathcal{A}}_{\lambda}^{\theta}(n, r)\right)$ is the direct sum of quantizations of products of Hilbert schemes. The morphism $\prod \operatorname{Hilb}_{n_{i}}\left(\mathbb{C}^{2}\right) \rightarrow \prod\left(\mathbb{C}^{2 n_{i}} / \mathfrak{S}_{n}\right)$ is an isomorphism over the non-vanishing locus of $\delta$. This implies that (5.4) is an isomorphism.

By the construction, (5.3) is the composition of $\mathrm{C}_{\nu_{0}}\left(\overline{\mathcal{A}}_{\lambda}(n, r)\left[\delta^{-1}\right]\right) \rightarrow \Gamma\left(\mathrm{C}_{\nu_{0}}\left(\overline{\mathcal{A}}_{\lambda}^{\theta}(n, r)\right)\right)\left[\delta^{-1}\right]$ and (5.4). So we have proved that $\mathrm{C}_{\nu_{0}}\left(\overline{\mathcal{A}}_{\lambda}(n, r)\right)\left[\delta^{-1}\right] \rightarrow \Gamma\left(\mathrm{C}_{\nu_{0}}\left(\overline{\mathcal{A}}_{\lambda}^{\theta}(n, r)\right)\right)\left[\delta^{-1}\right]$ is an isomorphism. 
Step \%. For $p \in \overline{\mathcal{M}}^{\theta}(n, r)^{T}$, let $L^{0}(p)$ be the corresponding irreducible $\Gamma\left(C_{\nu_{0}}\left(\overline{\mathcal{A}}_{\lambda}^{\theta}(n, r)\right)\right)$ module from category $\mathcal{O}$. These modules are either finite dimensional (those are parameterized by the multi-partitions with one part equal to $(n)$ and others empty) or has support of maximal dimension. It follows from Proposition 5.4 that all finite dimensional $L^{0}(p)$ restrict to pairwise non-isomorphic $\mathrm{C}_{\nu_{0}}\left(\overline{\mathcal{A}}_{\lambda}(n, r)\right)$-modules. Now consider $L^{0}(p)$ with support of maximal dimension. We claim that the localizations $L^{0}(p)\left[\delta^{-1}\right]$ are pairwise non-isomorphic simple $\Gamma\left(\mathrm{C}_{\nu_{0}}\left(\overline{\mathcal{A}}_{\lambda}^{\theta}(n, r)\right)\right)\left[\delta^{-1}\right]$-modules. Let us consider $p=\left(p^{1}, \ldots, p^{r}\right)$ and $p^{\prime}=\left(p^{\prime 1}, \ldots, p^{\prime r}\right)$ with $\left|p^{i}\right|=\left|p^{i}\right|$ for all $i$ and show that

- the localizations of $L^{0}(p), L^{0}\left(p^{\prime}\right)$ are simple

- and, moreover, are isomorphic only if $p=p^{\prime}$.

The analogous claims hold if we localize to the regular locus for $\prod_{i=1}^{r} \mathfrak{S}_{\left|p^{i}\right|}$. Indeed, this localization realizes the $\mathrm{KZ}$ functor that is a quotient onto its image. So the images of $L^{0}(p), L^{0}\left(p^{\prime}\right)$ under this localization are simple and non-isomorphic. The modules $L^{0}(p)\left[\delta^{-1}\right], L^{0}\left(p^{\prime}\right)\left[\delta^{-1}\right]$ further restrict to the locus where $x_{i} \neq x_{j}$ for all $i, j$. But there is no monodromy of the D-modules $L^{0}(p)\left[\delta^{-1}\right], L^{0}\left(p^{\prime}\right)\left[\delta^{-1}\right]$ along those additional hyperplanes and these D-modules have regular singularities everywhere. It follows that they remain simple and nonisomorphic (if $p \neq p^{\prime}$ ).

Step 8. So we see that the $\mathrm{C}_{\nu_{0}}\left(\overline{\mathcal{A}}_{\lambda}(n, r)\right)\left[\delta^{-1}\right]$-modules $L^{0}(p)\left[\delta^{-1}\right]$ are simple and pairwise non-isomorphic. The $\mathrm{C}_{\nu_{0}}\left(\overline{\mathcal{A}}_{\lambda}(n, r)\right)$-module $L^{0}(p)$ is finitely generated, because the algebra $\Gamma\left(\mathrm{C}_{\nu_{0}}\left(\overline{\mathcal{A}}_{\lambda}^{\theta}(n, r)\right)\right)$ is a finitely generated $\mathrm{C}_{\nu_{0}}\left(\overline{\mathcal{A}}_{\lambda}(n, r)\right)$-module, the latter follows from [L5, Lemma 5.4]. So the $\mathrm{C}_{\nu_{0}}\left(\overline{\mathcal{A}}_{\lambda}(n, r)\right)$-module $L^{0}(p)$ lies in $\mathcal{O}_{\nu}\left(\mathrm{C}_{\nu_{0}}\left(\overline{\mathcal{A}}_{\lambda}(n, r)\right)\right)$ thanks to the weight decomposition. There is a simple constituent $\underline{L}^{0}(p)$ of the $\mathrm{C}_{\nu_{0}}\left(\overline{\mathcal{A}}_{\lambda}(n, r)\right)$ module $L^{0}(p)$ with $\underline{L}^{0}(p)\left[\delta^{-1}\right]=L^{0}(p)\left[\delta^{-1}\right]$ because the right hand side is simple. The finite dimensional modules $L^{0}(p)$ together with the modules of the form $\underline{L}^{0}(p)$ give a required number of pairwise nonisomorphic simple $\mathrm{C}_{\nu_{0}}\left(\overline{\mathcal{A}}_{\lambda}(n, r)\right)$-modules.

5.4. Completion of proofs. The following proposition completes the proof of Theorem 1.1 .

Proposition 5.6. Let $\lambda$ be a positive parameter with denominator $n$. Then abelian localization holds for $(\lambda$, det).

Proof. The proof is in several steps.

Step 1. Since $\Gamma_{\lambda}^{\theta}: \mathcal{O}_{\nu}\left(\overline{\mathcal{A}}_{\lambda}^{\theta}(n, r)\right) \rightarrow \mathcal{O}_{\nu}\left(\overline{\mathcal{A}}_{\lambda}(n, r)\right)$ is a quotient functor, to prove that it is an equivalence it is enough to verify that the number of simples in these two categories is the same. The number of simples in $\mathcal{O}_{\nu}\left(\overline{\mathcal{A}}_{\lambda}(n, r)\right)$ coincides with that for $\mathcal{O}\left(C_{\nu_{0}}\left(\overline{\mathcal{A}}_{\nu}(n, r)\right)\right)$ by the paragraph before Proposition 5.5. The latter is bigger than or equal to the number of simples for $\mathcal{O}\left(\bigoplus \overline{\mathcal{A}}_{\lambda}\left(n_{1}, \ldots, n_{r} ; r\right)\right)$ that, in its turn coincides with the number of the $r$ multipartitions of $n$ because abelian localization holds for all summands $\overline{\mathcal{A}}_{\lambda}\left(n_{1}, \ldots, n_{r} ; r\right)$. We deduce that the number of simples in $\mathcal{O}_{\nu}\left(\overline{\mathcal{A}}_{\lambda}^{\theta}(n, r)\right)$ and in $\mathcal{O}_{\nu}\left(\overline{\mathcal{A}}_{\lambda}(n, r)\right)$ coincide. So we see that $\Gamma_{\lambda}^{\theta}: \mathcal{O}_{\nu}\left(\overline{\mathcal{A}}_{\lambda}^{\theta}(n, r)\right) \rightarrow \mathcal{O}_{\nu}\left(\overline{\mathcal{A}}_{\lambda}(n, r)\right)$ is an equivalence. Now we are going to show that this implies that $\Gamma_{\lambda}^{\theta}: \overline{\mathcal{A}}_{\lambda}^{\theta}(n, r)-\bmod \rightarrow \overline{\mathcal{A}}_{\lambda}(n, r)$-mod is an equivalence. Below we write $\mathcal{O}$ instead of $\mathcal{O}_{\nu}$. Our argument is similar to the proof of [L4, Theorem 2.1].

Step 2. Since $\Gamma_{\lambda}^{\theta}$ is an equivalence between the categories $\mathcal{O}$, we see that $\overline{\mathcal{A}}_{\lambda, \chi}^{0}(n, r) \otimes_{\overline{\mathcal{A}}_{\lambda}(n, r)}$ - and $\overline{\mathcal{A}}_{\lambda+\chi,-\chi}^{0}(n, r) \otimes_{\overline{\mathcal{A}}_{\lambda+\chi}(n, r)} \bullet$ are mutually inverse equivalences between $\mathcal{O}\left(\overline{\mathcal{A}}_{\lambda}(n, r)\right)$ and $\mathcal{O}\left(\overline{\mathcal{A}}_{\lambda+\chi}(n, r)\right)$ for all $\lambda>0$ and $\chi \in \mathbb{Z}$ such that $(\lambda+\chi$, det $)$ satisfies abelian localization (which happens as long as $\chi$ is sufficiently large). Set $\mathcal{B}:=\overline{\mathcal{A}}_{\lambda+\chi,-\chi}^{0}(n, r) \otimes_{\overline{\mathcal{A}}_{\lambda+\chi}(n, r)}$ $\overline{\mathcal{A}}_{\lambda, \chi}^{0}(n, r)$. This is a $\mathrm{HC} \overline{\mathcal{A}}_{\lambda}(n, r)$-bimodule with a natural homomorphism to $\overline{\mathcal{A}}_{\lambda}(n, r)$ 
such that the induced homomorphism $\mathcal{B} \otimes_{\overline{\mathcal{A}}_{\lambda}(n, r)} M \rightarrow M$ is an isomorphism for any $M \in \mathcal{O}\left(\overline{\mathcal{A}}_{\lambda}(n, r)\right)$. On the other hand, for any nonzero $x \in \overline{\mathcal{M}}(n, r)$, the bimodules $\overline{\mathcal{A}}_{\lambda+\chi,-\chi}^{0}(n, r), \overline{\mathcal{A}}_{\lambda, \chi}^{0}(n, r)$ are mutually inverse Morita equivalences, this follows from Lemma 3.10 since abelian localization holds for all slice algebras with parameters whose denominator is $n$. It follows that both kernel and cokernel of $\mathcal{B} \rightarrow \overline{\mathcal{A}}_{\lambda}(n, r)$ are finite dimensional.

Step 3. Let $L$ denote an irreducible finite dimensional $\overline{\mathcal{A}}_{\lambda}(n, r)$-module, it is unique because of the equivalence $\mathcal{O}\left(\overline{\mathcal{A}}_{\lambda}(n, r)\right) \cong \mathcal{O}\left(\overline{\mathcal{A}}_{\lambda+\chi}(n, r)\right)$ and Proposition 4.1, Since the homomorphism $\mathcal{B} \otimes_{\overline{\mathcal{A}}_{\lambda}(n, r)} L \rightarrow L$ is an isomorphism, we see that $\mathcal{B} \rightarrow \overline{\mathcal{A}}_{\lambda}(n, r)$. Let $K$ denote the kernel. We have an exact sequence

$$
\operatorname{Tor}_{\overline{\mathcal{A}}_{\lambda}(n, r)}^{1}\left(\overline{\mathcal{A}}_{\lambda}(n, r), L\right) \rightarrow K \otimes_{\overline{\mathcal{A}}_{\lambda}(n, r)} L \rightarrow \mathcal{B} \otimes_{\overline{\mathcal{A}}_{\lambda}(n, r)} L \rightarrow L \rightarrow 0
$$

Clearly, the first term is zero, while the last homomorphism is an isomorphism. We deduce that $K \otimes_{\overline{\mathcal{A}}_{\lambda}(n, r)} L=0$. But $K$ is a finite dimensional $\overline{\mathcal{A}}_{\lambda}(n, r)$-bimodule and hence a $\overline{\mathcal{A}}_{\lambda}(n, r) /$ Ann $L$-bimodule. So its tensor product with $L$ can only be zero if $K=0$.

Step 4. So we see that $\overline{\mathcal{A}}_{\lambda+\chi,-\chi}^{0}(n, r) \otimes_{\overline{\mathcal{A}}_{\lambda+\chi}(n, r)} \overline{\mathcal{A}}_{\lambda, \chi}^{0}(n, r) \cong \overline{\mathcal{A}}_{\lambda}(n, r)$. Similarly, $\overline{\mathcal{A}}_{\lambda, \chi}^{0}(n, r) \otimes_{\overline{\mathcal{A}}_{\lambda}(n, r)} \overline{\mathcal{A}}_{\lambda+\chi,-\chi}^{0}(n, r) \cong \overline{\mathcal{A}}_{\lambda+\chi}(n, r)$. It follows that $\Gamma_{\lambda}^{\theta}$ is an equivalence between $\overline{\mathcal{A}}_{\lambda}^{\theta}(n, r)-\bmod$ and $\overline{\mathcal{A}}_{\lambda}(n, r)-\bmod$.

Proof of Theorem 1.2. Let $L$ denote a finite dimensional irreducible representation of

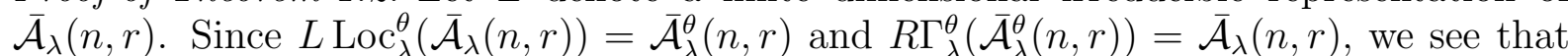
$R \Gamma_{\lambda}^{\theta} \circ L \operatorname{Loc}_{\lambda}^{\theta}$ is the identity functor of $D^{-}\left(\overline{\mathcal{A}}_{\lambda}(n, r)\right.$-mod). The homology of $L \operatorname{Loc}_{\lambda}^{\theta}(L)$ are supported on $\bar{\rho}^{-1}(0)$. From Proposition 4.1 it follows that the denominator of $\lambda$ is $n$. Thanks to that proposition combined with Theorem 1.1, the present proof reduces to showing that for $\lambda$ between $-r$ and 0 and with denominator $n$, the algebra $\overline{\mathcal{A}}_{\lambda}(n, r)$ has no finite dimensional representations.

Recall that $\Gamma_{\lambda}^{\theta}$ is an exact functor. Since $R \Gamma_{\lambda}^{\theta} \circ L \operatorname{Loc}_{\lambda}^{\theta}$ is the identity, the functor $\Gamma_{\lambda}^{\theta}$ does not kill the simple $\overline{\mathcal{A}}_{\lambda}^{\theta}(n, r)$-module $\tilde{L}$ supported on $\bar{\rho}^{-1}(0)$. On the other hand, $\Gamma_{\lambda}^{\theta}$ does not kill modules whose support intersects $\overline{\mathcal{M}}^{\theta}(n, r)^{r e g}$, the open subvariety in $\overline{\mathcal{M}}^{\theta}(n, r)$, where $\bar{\rho}$ is an isomorphism. In fact, every simple in $\mathcal{O}\left(\overline{\mathcal{A}}_{\lambda}^{\theta}(n, r)\right)$ is either supported on $\bar{\rho}^{-1}(0)$ (if it is homologically shifted under the wall-crossing functor so that the global sections are finite dimensional) or its support intersects $\overline{\mathcal{M}}^{\theta}(n, r)^{\text {reg }}$ (if it is not).

So we see that $\Gamma_{\lambda}^{\theta}$ does not kill any irreducible module in $\mathcal{O}\left(\overline{\mathcal{A}}_{\lambda}^{\theta}(n, r)\right)$. So it is an equivalence. By the proof of Proposition [5.6, $(\lambda, \theta)$ satisfies abelian localization, which is impossible as we already know.

\section{TWO-SIDED IDEALS AND DIMENSIONS OF SUPPORTS}

6.1. Two-sided ideals. The goal of this section is to prove Theorem 1.3. We use the following notation. We write $\mathcal{A}$ for $\overline{\mathcal{A}}_{\lambda}(n, r)$ (where $\lambda$ is not of the form $\frac{s}{n^{\prime}}$ with $n^{\prime} \leqslant n$ coprime to $s$ and $\left.-r n^{\prime}<s<0\right)$ and write $\underline{\mathcal{A}}$ for $\overline{\mathcal{A}}_{\lambda}\left(n^{\prime}, r\right)$.

Let us start with the description of the two-sided ideals in $\underline{\mathcal{A}}$.

Lemma 6.1. There is a unique proper ideal in $\underline{\mathcal{A}}$.

Proof. The proper slice algebras for $\underline{\mathcal{A}}$ have no finite dimensional representations, compare to the proof of Proposition 4.1. So every ideal $\mathcal{J} \subset \underline{\mathcal{A}}$ is either of finite codimension or $\mathrm{V}(\underline{\mathcal{A}} / \mathcal{J})=\overline{\mathcal{M}}\left(n^{\prime}, r\right)$. The algebra $\underline{\mathcal{A}}$ has no zero divisors so the second option is only possible when $\mathcal{J}=\{0\}$. Now suppose that $\mathcal{J}$ is of finite codimension. Then $\underline{\mathcal{A}} / \mathcal{J}$ (viewed 
as a left $\underline{\mathcal{A}}$-module) is the sum of several copies of the finite dimensional irreducible $\underline{\mathcal{A}}$ module. So $\mathcal{J}$ coincides with the annihilator of the finite dimensional irreducible module, and we are done.

Let $\underline{\mathcal{J}}$ denote the unique two-sided ideal.

Now we are going to describe the two-sided ideals in $\underline{\mathcal{A}}^{\otimes k}$. For this we need some notation. Set $\underline{\mathcal{I}}_{i}:=\underline{\mathcal{A}}^{\otimes i-1} \otimes \underline{\mathcal{J}} \otimes \underline{\mathcal{A}}^{\otimes k-i-1}$. For a subset $\Lambda \subset\{1, \ldots, k\}$ define the ideals $\underline{\mathcal{I}}_{\Lambda}:=\sum_{i \in \Lambda} \underline{\mathcal{I}}_{i}, \underline{\mathcal{I}}^{\Lambda}:=\prod_{i \in \Lambda} \underline{\mathcal{I}}_{i}$.

Recall that a collection of subsets in $\{1, \ldots, k\}$ is called an anti-chain if none of these subsets is contained in another. Also recall that an ideal $I$ in an associative algebra $A$ is called semi-prime if it is the intersection of prime ideals.

Lemma 6.2. The following is true.

(1) The prime ideals in $\underline{\mathcal{A}}^{\otimes k}$ are precisely the ideals $\underline{\mathcal{I}}_{\Lambda}$.

(2) For every ideal $\mathcal{I} \subset \underline{\mathcal{A}}^{\otimes k}$, there is a unique anti-chain $\Lambda_{1}, \ldots, \Lambda_{q}$ of subsets in $\{1, \ldots, k\}$ such that $\mathcal{I}=\bigcap_{i=1}^{p} \mathcal{I}_{\Lambda_{i}}$. In particular, every ideal is semi-prime.

(3) For every ideal $\mathcal{I} \subset \underline{\mathcal{A}}^{\otimes k}$, there is a unique anti-chain $\Lambda_{1}^{\prime}, \ldots, \Lambda_{q}^{\prime}$ of subsets of $\{1, \ldots, k\}$ such that $\mathcal{I}=\sum_{i=1}^{q} \mathcal{I}^{\Lambda_{i}^{\prime}}$.

(4) The anti-chains in (2) and (3) are related as follows: from an antichain in (2), we form all possible subsets containing an element from each of $\Lambda_{1}, \ldots, \Lambda_{p}$. The minimal such subsets form an anti-chain in (3).

The proof essentially appeared in [L1, 5.8].

Proof. Let us prove (1). Let $\mathcal{I}$ be a prime ideal. Let $x$ be a generic point in an open leaf $\mathcal{L} \subset \mathrm{V}\left(\underline{\mathcal{A}}^{\otimes k} / \mathcal{I}\right)$ of maximal dimension. The corresponding slice algebra $\underline{\mathcal{A}}^{\prime}$ has a finite dimensional representation and so is again the product of several copies of $\underline{\mathcal{A}}$. The leaf $\mathcal{L}$ is therefore the product of one-point leaves and open leaves in $\overline{\mathcal{M}}\left(n^{\prime}, r\right)^{k}$. An irreducible finite dimensional representation of $\underline{\mathcal{A}}^{\prime}$ is unique, let $\mathcal{I}^{\prime}$ be its annihilator.

Consider the categories $\mathrm{HC}_{\overline{\mathcal{L}}}\left(\underline{\mathcal{A}}^{\otimes k}\right)$ of all HC$\underline{\mathcal{A}}^{\otimes k}$-bimodules whose associated variety is contained in $\overline{\mathcal{L}}$ and $\mathrm{HC}_{\text {fin }}\left(\mathcal{A}^{\prime}\right)$ of finite dimensional $\underline{\mathcal{A}}^{\prime}$-bimodules (that are automatically $\mathrm{HC})$. So the functor $\bullet_{\dagger, x}$ restricts to $\mathrm{HC}_{\overline{\mathcal{L}}}\left(\underline{\mathcal{A}}^{\otimes k}\right) \rightarrow \mathrm{HC}_{\text {fin }}\left(\underline{\mathcal{A}}^{\prime}\right)$. As we have mentioned in Section 3.4, this functor admits a right adjoint

$$
\bullet^{\dagger, x}: \mathrm{HC}_{f i n}\left(\underline{\mathcal{A}}^{\prime}\right) \rightarrow \mathrm{HC}_{\overline{\mathcal{L}}}\left(\underline{\mathcal{A}}^{\otimes k}\right) \text {. }
$$

Let $\mathcal{I}^{1}$ denote the kernel of the natural homomorphism $\underline{\mathcal{A}}^{\otimes k} \rightarrow\left(\underline{\mathcal{A}^{\prime}} / \mathcal{I}^{\prime}\right)^{\dagger, x}$ and $\mathcal{I} \subset \mathcal{I}^{1}$. So $\mathrm{V}\left(\underline{\mathcal{A}}^{\otimes k} / \mathcal{I}^{1}\right)=\overline{\mathcal{L}}$. It follows from $\left[\mathrm{BoKr}\right.$, Corollar 3.6] that $\mathcal{I}=\overline{\mathcal{I}}^{1}$. So the number of the prime ideals coincides with that of the non-empty subsets $\{1, \ldots, k\}$. On the other hand, the ideals $\mathcal{I}^{\Lambda}$ are all different (they have different associated varieties) and all prime (the quotient $\underline{\mathcal{A}}^{\otimes k} / \mathcal{I}^{\Lambda}$ is the product of a matrix algebra and the algebra $\underline{\mathcal{A}}^{\otimes k-|\Lambda|}$ that has no zero divisors).

Let us prove (2) (and simultaneously (3)). Let us write $\mathcal{I}_{\Lambda_{1}, \ldots, \Lambda_{p}}$ for $\bigcap_{j=1}^{s} \mathcal{I}_{\Lambda_{j}}$. For ideals in $\underline{\mathcal{A}}^{\otimes k-1}$ we use notation like $\underline{\mathcal{I}}_{\Lambda_{1}^{\prime}, \ldots, \Lambda_{q}^{\prime}}$. Reordering the indexes, we may assume that $k \in \Lambda_{1}, \ldots, \Lambda_{s}$ and $k \notin \Lambda_{s+1}, \ldots, \Lambda_{p}$. Set $\Lambda_{j}^{\prime}:=\Lambda_{j} \backslash\{k\}$ for $j \leqslant s$. Then

$$
\mathcal{I}_{\Lambda_{1}, \ldots, \Lambda_{p}}=\left(\underline{\mathcal{A}}^{\otimes k-1} \otimes \underline{\mathcal{J}}+\underline{\mathcal{I}}_{\Lambda_{1}^{\prime}, \ldots, \Lambda_{s}^{\prime}} \otimes \underline{\mathcal{A}}\right) \cap\left(\underline{\mathcal{I}}_{\Lambda_{s+1}, \ldots, \Lambda_{p}} \otimes \underline{\mathcal{A}}\right) \text {. }
$$

We claim that the right hand side of (6.1) coincides with

$$
\underline{\mathcal{I}}_{\Lambda_{s+1}, \ldots, \Lambda_{p}} \otimes \underline{\mathcal{J}}+\underline{\mathcal{I}}_{\Lambda_{1}^{\prime}, \ldots, \Lambda_{s}^{\prime}, \Lambda_{s+1}, \ldots, \Lambda_{p}} \otimes \underline{\mathcal{A}} .
$$


First of all, we notice that $(6.2)$ is contained in (6.1). So we only need to prove the opposite inclusion. The projection of (6.1) to $\underline{\mathcal{A}}^{\otimes k-1} \otimes(\underline{\mathcal{A}} / \underline{\mathcal{J}})$ is contained in $\underline{\mathcal{I}}_{\Lambda_{1}^{\prime}, \ldots, \Lambda_{s}^{\prime}, \Lambda_{s+1}, \ldots, \Lambda_{p}}$ and hence also in the projection of (6.2). Also the intersection of (6.1) with $\underline{\mathcal{A}}^{\otimes k-1} \otimes \underline{\mathcal{J}}$ is contained in $\underline{\mathcal{I}}_{\Lambda_{s+1}, \ldots, \Lambda_{p}} \otimes \underline{\mathcal{J}}$. So (6.1) is included into (6.2).

Repeating this argument with the sum similar to (6.2) but for other $k-1$ factors of $\underline{\mathcal{A}}^{\otimes k}$ we conclude that $\mathcal{I}_{\Lambda_{1}, \ldots, \Lambda_{p}}=\sum_{j} \mathcal{I}^{\Lambda_{j}^{\prime}}$, where the subsets $\Lambda_{j}^{\prime} \subset\{1, \ldots, k\}$ are formed as described in (4). So we see that the ideals (2) are the same as the ideals in (3) and that (4) holds. What remains to do is to prove that every ideal has the form described in (2). To start with, we notice that every semi-prime ideal has the form as in (2) because of (1). In particular, the radical of any ideal has such form.

Clearly, $\mathcal{I}^{\Lambda_{1}^{\prime}} \mathcal{I}_{2}^{\Lambda_{2}^{\prime}}=\mathcal{I}^{\Lambda_{1}^{\prime} \cup \Lambda_{2}^{\prime}}$. So it follows that any sum of the ideals $\mathcal{I}^{\Lambda_{j}^{\prime}}$ coincides with its square. So if $\mathcal{I}$ is an ideal whose radical is $\mathcal{I}_{\Lambda_{1}, \ldots, \Lambda_{p}}$, then $\mathcal{I}$ coincides with its radical. This completes the proof.

Now we are ready to establish a result that will imply Theorem 1.3 for nonsingular parameters $\lambda$. Let $x_{i} \in \overline{\mathcal{M}}(n, r)$ be a point corresponding to the leaf with slice $\overline{\mathcal{M}}\left(n^{\prime}, r\right)^{i}$ (i.e. to the semisimple representations of the form $\left.r^{0} \oplus\left(r^{1}\right)^{n^{\prime}} \oplus \ldots \oplus\left(r^{i}\right)^{n^{\prime}}\right)$. We set $\left.\mathcal{J}_{i}=\operatorname{ker}\left[\mathcal{A} \rightarrow(\underline{\mathcal{A}} / \underline{\mathcal{I}})^{\otimes i}\right)^{\dagger, x_{i}}\right]$.

Proposition 6.3. The ideals $\mathcal{J}_{i}, i=1, \ldots, q$, have the following properties.

(1) The ideal $\mathcal{J}_{i}$ is prime for any $i$.

(2) $\mathrm{V}\left(\mathcal{A} / \mathcal{J}_{i}\right)=\overline{\mathcal{L}}_{i}$, where $\mathcal{L}_{i}$ is the symplectic leaf containing $x_{i}$.

(3) $\mathcal{J}_{1} \subsetneq \mathcal{J}_{2} \subsetneq \ldots \subsetneq \mathcal{J}_{q}$

(4) Any proper two-sided ideal in $\mathcal{A}$ is one of $\mathcal{J}_{i}$.

Proof. (2) follows from the construction. Also from the construction it follows that $\mathcal{J}_{i}$ is the maximal among ideal with given associated variety. So it is prime similarly to the proof of (1) of Lemma 6.2.

Let us prove $(3)$. Since $\left(\mathcal{J}_{i}\right)_{\dagger, x_{i}}$ has finite codimension, we see that it coincides with the maximal ideal in $\underline{\mathcal{A}}^{\otimes i}$. So $\left(\mathcal{J}_{j}\right)_{\dagger, x_{i}} \subset\left(\mathcal{J}_{i}\right)_{\dagger, x_{i}}$ for $j<i$. Again from the construction of $\mathcal{J}_{i}$, it follows that $\mathcal{J}_{j} \subsetneq \mathcal{J}_{i}$.

Let us prove (4). The functor $\bullet_{\dagger, x_{q}}$ is faithful. Indeed, otherwise we have a HC bimodule $\mathcal{M}$ with $\mathrm{V}(\mathcal{M}) \cap \mathcal{L}_{q}=\varnothing$. But $\mathcal{M}_{\dagger, x}$ has to be nonzero finite dimensional for some $x$ and this is only possible when $x \in \mathcal{L}_{i}$ for some $i$. But $\mathcal{L}_{q} \subset \overline{\mathcal{L}}_{i}$ for all $i$ that shows faithfulness. Since $\bullet_{\dagger, x_{q}}$ is faithful and exact, it follows that it embeds the lattice of the ideals in $\mathcal{A}$ into that in $\underline{\mathcal{A}}^{\otimes q}$. We claim that this implies that every ideal in $\mathcal{A}$ is semiprime. Indeed, the functor $\bullet_{\dagger, x_{q}}$ is, in addition, tensor and so preserves products of ideals. In particular, any two-sided ideal in $\mathcal{A}$ coincides with its square. Our claim follows from (2) of Lemma 6.2. But every prime ideal in $\mathcal{A}$ is some $\mathcal{J}_{i}$, this is proved analogously to (1) of Lemma 6.2. Since the ideals $\mathcal{J}_{i}$ form a chain, any semiprime ideal is prime and so coincides with some $\mathcal{J}_{i}$.

Proof. The case of finite homological dimension follows from Proposition 6.3, Let us now consider the case when $\lambda=\frac{s}{n^{\prime}}$ with $-r n^{\prime}<s<0$. The algebra $\overline{\mathcal{A}}_{\lambda}(n, r)$ has no finite dimensional representations and neither does any of the slice algebras $\bigotimes_{i=1}^{k} \overline{\mathcal{A}}_{\lambda}\left(n_{i}, r\right)$. By using the restriction functors (say similarly to Step 3 of the proof of Proposition 4.1) we see that the algebra $\overline{\mathcal{A}}_{\lambda}(n, r)$ is simple. 
6.2. Restriction functors for asymptotic chamber. In this section we assume that $\nu$ is dominant meaning that $\nu(t)=\left(\operatorname{diag}\left(t^{d_{1}}, \ldots, t^{d_{r}}\right), t\right)$ with $d_{1} \gg d_{2} \gg \ldots \gg d_{r}$. We also assume that $\lambda>0$ and is Zariski generic.

Let $\tau=\left(\tau_{1}, \ldots, \tau_{k}\right)$ be a partition of $n$. Set $\mathcal{O}_{\nu, \lambda}(\tau, r):=\bigotimes_{i=1}^{k} \mathcal{O}_{\nu}\left(\mathcal{A}_{\lambda}\left(\tau_{i}, r\right)\right)$. We will produce exact functors $\operatorname{Res}_{\tau}: \mathcal{O}_{\nu, \lambda}(n, r) \rightarrow \mathcal{O}_{\nu, \lambda}(\tau, r)$ generalizing the BezrukavnikovEtingof functors, $\mathrm{BE}$, for $r=1$.

The filtration by the order of differential operators on $D(R)$ induces a filtration on $\mathcal{A}_{\lambda}(n, r)$. The degree zero part is $\mathbb{C}[R]^{G}=\mathbb{C}[\overline{\mathfrak{g}}]^{G}$. Pick a point $b \in \overline{\mathfrak{g}} / / G$ such that the stabilizer of the corresponding closed orbit is $\prod_{i=1}^{k} \mathrm{GL}\left(\tau_{i}\right)$ and consider the tensor $\mathcal{A}_{\lambda}(n, r)^{\wedge_{b}}:=\mathbb{C}[\overline{\mathfrak{g}} / / G]^{\wedge_{b}} \otimes_{\mathbb{C}[\overline{\mathfrak{g}}]^{G}} \mathcal{A}_{\lambda}(n, r)$. Since the adjoint action of $\mathbb{C}[\overline{\mathfrak{g}}]^{G}$ is locally nilpotent, $\mathcal{A}_{\lambda}(n, r)^{\wedge_{b}}$ is naturally an algebra. Set $\mathcal{A}_{\lambda}(\tau, r)^{\wedge_{0}}:=\bigotimes_{i=1}^{k} \mathcal{A}_{\lambda}\left(\tau_{i}, r\right)^{\wedge_{0}}$.

Lemma 6.4. We have a $\mathrm{GL}(r)$-equivariant isomorphism of filtered algebras $\vartheta: \mathcal{A}_{\lambda}(n, r)^{\wedge_{b} \stackrel{\sim}{\rightarrow}}$ $\bigotimes_{i=1}^{k} \mathcal{A}_{\lambda}\left(\tau_{i}, r\right)^{\wedge_{0}}$ (the action on the right hand side is diagonal).

Proof. Consider the Rees algebras $\mathcal{A}_{\lambda \hbar}(n, r), \mathcal{A}_{\lambda \hbar}(\tau, r)$ and their full completions $\mathcal{A}_{\lambda \hbar}(n, r)^{\wedge_{b}}$ (at the point with closed $G$-orbit given by a diagonal matrix in $\mathfrak{g} \subset \mu^{-1}(0)$ corresponding to $b)$ and $\mathcal{A}_{\lambda \hbar}(\tau, r)^{\wedge_{0}}$. As was mentioned in Section [3.3, see (3.2), we have a $\mathbb{C}[\hbar]$-linear isomorphism $\mathcal{A}_{\lambda \hbar}(n, r)^{\wedge_{b}} \cong \mathcal{A}_{\lambda \hbar}(\tau, r)^{\wedge_{0}}$ that can be made $\operatorname{GL}(r) \times \mathbb{C}^{\times}$-equivariant (here $\mathbb{C}^{\times}$is the contracting action) because we complete at the $\operatorname{GL}(r) \times \mathbb{C}^{\times}$-stable points. By taking $\mathbb{C}^{\times}$-finite parts and taking the quotients by $\hbar-1$ we get an isomorphism in the lemma.

We will use an isomorphism from Lemma 6.4 to produce a functor $\operatorname{Res}_{\tau}$. First we need to establish an equivalence of the category $\mathcal{O}_{\nu, \lambda}(\tau, r)$ with a certain category of $\mathcal{A}_{\lambda}(\tau, r)^{\wedge_{0}}$-modules. As usual, set $\nu_{0}(t)=\left(\operatorname{diag}\left(t^{d_{1}}, \ldots, t^{d_{r}}\right), 1\right)$. We consider the category $\mathcal{O}_{\nu, \lambda}(\tau, r)^{\wedge_{0}}$ consisting of all finitely generated $\mathcal{A}_{\lambda}(\tau, r)^{\wedge_{0}}$-modules such that $h_{0}=d_{1} \nu_{0}$ acts locally finitely with eigenvalues bounded from above and generalized eigenspaces that are finitely generated over $\mathbb{C}\left[\mathfrak{g}_{\tau} / / G_{\tau}\right]^{\wedge_{0}}$, where $\mathfrak{g}_{\tau}$ is the standard Levi subalgebra of $\mathfrak{g}$ corresponding to $\tau$. Note that all generalized $h_{0}$-eigenspaces in a module from $\mathcal{O}_{\nu, \lambda}(n, r)$ are finitely generated over $\mathbb{C}\left[\mathfrak{g}_{\tau}\right]^{G_{\tau}}$. So we get an exact functor

$$
N \mapsto N^{\wedge_{0}}:=\mathbb{C}\left[\mathfrak{g}_{\tau} / / G_{\tau}\right]^{\wedge_{0}} \otimes_{\mathbb{C}\left[\mathfrak{g}_{\tau}\right] G_{\tau}} N: \mathcal{O}_{\nu^{+}, \lambda}(\tau, r) \rightarrow \mathcal{O}_{\nu, \lambda}(\tau, r)^{\wedge_{0}}
$$

Lemma 6.5. The functor $\bullet^{\wedge_{0}}$ is a category equivalence. A quasi-inverse functor is given by taking the $h$-finite elements, where $h$ is the image of 1 under the quantum comoment map for $t \mapsto \nu(t) \nu_{0}(t)^{-1}$.

Proof. Let $N_{\text {fin }}^{\prime}$ stand for the space of $h$-finite elements. It is easy to see that $N_{\text {fin }}^{\prime}$ is the sum of modules from $\mathcal{O}_{\nu, \lambda}(\tau, r)$. Note that all simultaneous generalized eigenspaces for $\left(h, h_{0}\right)$ are finite dimensional. This is because the generalized eigenspaces for $h_{0}$ are finitely generated modules over $\mathbb{C}\left[\mathfrak{g}_{\tau} / / G_{\tau}\right]^{\wedge_{0}}$ and the generalized eigenspaces for $h$ in such modules are finite dimensional. So $N_{\text {fin }}^{\prime}$ actually lies in $\mathcal{O}$. Also $N_{\text {fin }}^{\prime}$ is dense in $N^{\prime}$. Now the proof is easy.

We note that, for $M \in \mathcal{O}_{\nu}\left(\mathcal{A}_{\lambda}(n, r)\right)$, the $\mathcal{A}_{\lambda}(\tau, r)^{\wedge^{0}}$-module $\vartheta_{*}\left(\mathbb{C}[\mathfrak{g} / / G]^{\wedge_{b}} \otimes_{\mathbb{C}[\mathfrak{g}]^{G}} M\right)$ lies in $\mathcal{O}_{\nu, \lambda}(\tau, r)^{\wedge_{0}}$. We define $\operatorname{Res}_{\tau}(M)$ by $\left[\vartheta_{*}\left(\mathbb{C}[\mathfrak{g} / / G]^{\wedge_{b}} \otimes_{\mathbb{C}[\mathfrak{g}]} M\right)\right]_{\text {fin }}$. This is an exact functor by construction.

Now let us study properties of $\operatorname{Res}_{\tau}$.

First of all, the restriction functor behaves nicely on the level of associated varieties. The following result is a straightforward consequence of the construction. 
Lemma 6.6. The associated variety $\mathrm{V}\left(\operatorname{Res}_{\tau}(M)\right)$ is a unique conical (with respect to the contracting $\mathbb{C}^{\times}$-action) subvariety in $\mathcal{M}(\tau, r)$ such that $\mathrm{V}\left(\operatorname{Res}_{\tau}(M)\right) \cap \mathcal{M}(\tau, r)^{\wedge_{0}}=$ $\mathrm{V}(M) \cap \mathcal{M}(n, r)^{\wedge_{b}}$ (where we consider the full completions).

The following basic property is extremely important. We can consider the functor

$$
\underline{\operatorname{Res}}_{\tau}: \mathcal{O}_{\nu}\left(\mathrm{C}_{\nu_{0}}\left(\mathcal{A}_{\lambda}(n, r)\right)\right) \rightarrow \mathcal{O}_{\nu}\left(\mathrm{C}_{\nu_{0}}\left(\mathcal{A}_{\lambda}(\tau, r)\right)\right) \text {. }
$$

It is defined in the same way as $\operatorname{Res}_{\tau}$. On the summand corresponding to a composition $\mu=\left(n_{1}, \ldots, n_{r}\right)$, the functor $\underline{\operatorname{ReS}}_{\tau}$ coincides with the direct sum of suitable BezrukavnikovEtingof restriction functors. More precisely, the corresponding summand of $\mathrm{C}_{\nu_{0}}\left(\mathcal{A}_{\lambda}(n, r)\right)$ is $\mathcal{A}_{\lambda}\left(n_{1}, 1\right) \otimes \mathcal{A}_{\lambda+1}\left(n_{2}, 1\right) \otimes \ldots \otimes \mathcal{A}_{\lambda+r-1}\left(n_{r}, 1\right)$. Then

$$
\underline{\operatorname{Res}}_{\tau}(M)=\bigoplus_{S_{\mu} / W^{\prime}} \operatorname{Res}_{W^{\prime}}^{S_{\mu}} M,
$$

where the summation is the summation is taken over all $S_{\mu}$-orbits on $S / S_{\tau}$, here $W^{\prime}$ stands for the standard parabolic stabilizer of the orbit. We write $\operatorname{Res}_{W^{\prime}}^{S_{\mu}}$ for the BezrukavnikovEtingof restriction functor to the parabolic subgroup $W^{\prime}$ (or, more precisely, a version, where we do not change the space $\mathfrak{h}$ ).

We still have the Verma module functor $\Delta_{\nu_{0}}: \mathcal{O}_{\nu}\left(\mathrm{C}_{\nu_{0}}\left(\mathcal{A}_{\lambda}(\tau, r)\right)\right) \rightarrow \mathcal{O}_{\nu}\left(\mathrm{C}_{\nu_{0}}\left(\mathcal{A}_{\lambda}(n, r)\right)\right)$.

Lemma 6.7. We have isomorphisms of functors

$$
\Delta_{\nu_{0}} \circ \underline{\operatorname{Res}}_{\tau} \cong \operatorname{Res}_{\tau} \circ \Delta_{\nu_{0}}, \nabla_{\nu_{0}} \circ \underline{\operatorname{Res}}_{\tau} \cong \operatorname{Res}_{\tau} \circ \nabla_{\nu_{0}}
$$

Proof. Recall that by the construction, $\operatorname{Res}_{\tau}$ is isomorphic to the completion functor $\bullet^{\wedge}$.

Let us prove the first isomorphism in the lemma. Note first that

$$
\mathcal{A}_{\lambda}(n, r)^{\wedge_{b}} / \mathcal{A}_{\lambda}(n, r)^{\wedge_{b}}\left(\mathcal{A}_{\lambda}(n, r)^{\wedge_{b}}\right)^{>0, \nu_{0}} \cong \mathbb{C}[\mathfrak{g} / / G]^{\wedge_{b}} \otimes_{\mathbb{C}[\mathfrak{g}]^{G}}\left(\mathcal{A}_{\lambda}(n, r) / \mathcal{A}_{\lambda}(n, r) \mathcal{A}_{\lambda}(n, r)^{>0, \nu_{0}}\right)
$$

We can consider the functors in the first isomorphism as functors $\mathcal{O}_{\nu}\left(\mathrm{C}_{\nu_{0}}\left(\mathcal{A}_{\lambda}(n, r)\right)\right) \rightarrow$ $\mathcal{A}_{\lambda}(n, r)^{\wedge_{b}}$-mod. Then the right hand side is given by taking the tensor product over $\mathrm{C}_{\nu_{0}}\left(\mathcal{A}_{\lambda}(n, r)\right)$ with $\mathcal{A}_{\lambda}(n, r)^{\wedge_{b}} / \mathcal{A}_{\lambda}(n, r)^{\wedge_{b}}\left(\mathcal{A}_{\lambda}(n, r)^{\wedge_{b}}\right)^{>0, \nu_{0}}$. Since the isomorphism $\mathcal{A}_{\lambda}(n, r)^{\wedge_{b}} \cong$ $\mathcal{A}_{\lambda}(\tau, r)^{\wedge_{0}}$ is $\nu_{0}\left(\mathbb{C}^{\times}\right)$-equivariant, we get

$$
\mathcal{A}_{\lambda}(n, r)^{\wedge_{b}} / \mathcal{A}_{\lambda}(n, r)^{\wedge_{b}}\left(\mathcal{A}_{\lambda}(n, r)^{\wedge_{b}}\right)^{>0, \nu_{0}} \cong \mathcal{A}_{\lambda}(\tau, r)^{\wedge_{0}} / \mathcal{A}_{\lambda}(\tau, r)^{\wedge_{0}}\left(\mathcal{A}_{\lambda}(\tau, r)^{\wedge_{0}}\right)^{>0, \nu_{0}} .
$$

The bimodule in the right hand side coincides with

$$
\left(\mathcal{A}_{\lambda}(\tau, r) / \mathcal{A}_{\lambda}(\tau, r) \mathcal{A}_{\lambda}(\tau, r)^{>0, \nu_{0}}\right) \otimes_{\mathbb{C}\left[\mathfrak{g}_{\tau}\right]^{G}} \mathbb{C}\left[\mathfrak{g}_{\tau} / / G_{\tau}\right]^{\wedge_{0}} .
$$

It follows that the functor in the left hand side of the first isomorphism in the lemma is given by taking tensor product over $C_{\nu_{0}}\left(\mathcal{A}_{\lambda}(n, r)\right)$ with $\mathcal{A}_{\lambda}(\tau, r)^{\wedge_{0}} / \mathcal{A}_{\lambda}(\tau, r)^{\wedge_{0}}\left(\mathcal{A}_{\lambda}(\tau, r)^{\wedge_{0}}\right)^{>0, \nu_{0}}$. An isomorphism $\operatorname{Res}_{\tau} \circ \Delta_{\nu_{0}} \cong \Delta_{\nu_{0}} \circ \underline{\operatorname{ReS}}_{\tau}$.

Let us proceed to the second isomorphism. Similarly to the first one, both functors are isomorphic to

$$
\operatorname{Hom}_{\mathrm{C}_{\nu_{0}}\left(\mathcal{A}_{\lambda}(n, r)^{\wedge} b\right)}\left(\mathcal{A}_{\lambda}(n, r)^{\wedge_{b}} /\left(\mathcal{A}_{\lambda}(n, r)^{\wedge_{b}}\right)^{<0, \nu_{0}} \mathcal{A}_{\lambda}(n, r)^{\wedge_{b}}, \mathrm{C}_{\nu_{0}}\left(\mathcal{A}_{\lambda}(n, r)^{\wedge_{b}}\right) \otimes_{\mathrm{C}_{\nu_{0}}\left(\mathcal{A}_{\lambda}(n, r)\right)} \bullet\right),
$$

where we take the restricted Hom (with respect to the natural grading on the first argument).

For $M \in \mathcal{O}_{\nu}\left(\mathrm{C}_{\nu_{0}}\left(\mathcal{A}_{\lambda}(\tau, r)\right)\right)$ we write $L_{\nu_{0}}(M)$ for the maximal quotient of $\Delta_{\nu_{0}}(M)$ that does not intersect the highest weight subspace $M$. Equivalently, $L_{\nu_{0}}(M)$ is the image of the natural homomorphism $\Delta_{\nu_{0}}(M) \rightarrow \nabla_{\nu_{0}}(M)$ (induced by the identity map $M \rightarrow M$ ). 
The following corollary of Lemma 6.7 will play a crucial role in computing the annihilators of simple objects in $\mathcal{O}_{\nu, \lambda}(n, r)$.

Corollary 6.8. For $M \in \mathcal{O}_{\nu}\left(C_{\nu_{0}}\left(\mathcal{A}_{\lambda}(n, r)\right)\right)$, we have $\operatorname{Res}_{\tau}\left(L_{\nu_{0}}(M)\right) \cong L_{\nu_{0}}\left(\underline{\operatorname{Res}}_{\tau} M\right)$.

Proof. We can assume that $M \in \mathcal{O}_{\nu}\left(\left.\mathrm{C}_{\nu_{0}}\left(\mathcal{A}_{\lambda}(n, r)\right)\right|_{\tau^{\prime}}\right)$ (where $\left.\mathcal{A}_{\lambda}(n, r)\right)\left.\right|_{\mu}$ is the summand of $\mathrm{C}_{\nu_{0}}\left(\mathcal{A}_{\lambda}(n, r)\right)$ corresponding to a composition $\left.\mu\right)$. The natural map $\Delta_{\nu_{0}}(M) \rightarrow \nabla_{\nu_{0}}(M)$ gives rise to a map $\operatorname{Res}_{\tau} \circ \Delta_{\nu_{0}}(M) \rightarrow \operatorname{Res}_{\tau} \circ \nabla_{\nu_{0}}(M)$ that is the identity on the highest weight spaces for $h_{0}$. The identifications

$$
\operatorname{Res}_{\tau} \circ \Delta_{\nu_{0}}(M) \cong \Delta_{\nu_{0}} \circ \underline{\operatorname{Res}}_{\tau}(M), \operatorname{Res}_{\tau} \circ \nabla_{\nu_{0}}(M) \cong \nabla_{\nu_{0}} \circ \underline{\operatorname{Res}}_{\tau}(M)
$$

are the identity on the highest weight spaces, by the construction. So applying the functor $\operatorname{Res}_{\tau}$ to the morphism $\Delta_{\nu_{0}}(M) \rightarrow \nabla_{\nu_{0}}(M)$ we get the natural morphism $\Delta_{\nu_{0}} \circ \underline{\operatorname{Res}}_{\tau}(M) \rightarrow$ $\nabla_{\nu_{0}} \circ \underline{\operatorname{Res}}_{\tau}(M)$. Since $\operatorname{Res}_{\tau}$ is exact, our claim follows.

To finish this section let us study the interaction of $\operatorname{Res}_{\tau}$ with highest weight structures.

Proposition 6.9. The functor $\operatorname{Res}_{\tau}$ maps (co)standard objects to (co)standardly filtered ones, tiltings to tiltings, projectives to projectives, injectives to injectives.

Proof. The proof is in several steps.

Step 1. Let us show that $\operatorname{Res}_{\tau}$ maps standard objects to standardly filtered ones. Lemma 6.7 reduces this statement to showing that $\underline{\operatorname{Res}}_{\tau}$ maps standard objects to standardly filtered ones. This property of $\underline{\operatorname{Res}}_{\tau}$ follows from [S, Section 2].

Step 2. Similarly we see that $\operatorname{Res}_{\tau}$ maps costandard objects into costandardly filtered ones. Because of this, $\operatorname{Res}_{\tau}$ sends tiltings to tiltings.

Step 3. Let us show that $\operatorname{Res}_{\tau}$ maps injectives to injectives. The functor $\mathfrak{W J C}_{\lambda \leftarrow \lambda^{-}}=$ $\mathcal{A}_{\lambda^{-}, \lambda-\lambda^{-}}^{0}(n, r) \otimes_{\mathcal{A}_{\lambda^{-}}(n, r)}^{L} \bullet$ is a Ringel duality functor and hence induces equivalences $\mathcal{O}_{\nu}\left(\mathcal{A}_{\lambda^{-}}(n, r)\right)$-tilt $\stackrel{\sim}{\rightarrow} \mathcal{O}_{\nu}\left(\mathcal{A}_{\lambda}(n, r)\right)$-inj. It follows that $\mathcal{A}_{\lambda^{-}, \lambda-\lambda^{-}}^{0}(n, r) \otimes_{\mathcal{A}_{\lambda}^{-}(n, r)} \bullet$ gives this equivalence. On the other hand from the definition of $\operatorname{Res}_{\tau}$ we see that

$$
\operatorname{Res}_{\tau}\left(\mathcal{A}_{\lambda^{-}, \lambda-\lambda^{-}}^{0}(n, r) \otimes_{\mathcal{A}_{\lambda^{-}}(n, r)} \bullet\right) \cong \mathcal{A}_{\lambda^{-}, \lambda-\lambda^{-}}^{0}(n, r)_{\dagger, \tau} \otimes_{\mathcal{A}_{\lambda^{-}}(\tau ; r)} \operatorname{Res}_{\tau}(\bullet)
$$

But the bimodule $\mathcal{A}_{\lambda^{-}, \lambda-\lambda^{-}}^{0}(n, r)_{\dagger, \tau}$ is a wall-crossing bimodule for the algebras $\mathcal{A}_{?}(\tau ; r)$. So taking tensor product with this bimodule maps tiltings to injectives. So the right hand side of (6.4) maps tiltings to injectives. So does the left hand side. Since $\mathcal{A}_{\lambda^{-}, \lambda_{-} \lambda^{-}}^{0}(n, r) \otimes_{\mathcal{A}_{\lambda^{-}}(n, r)}$ - is an equivalence $\mathcal{O}_{\nu}\left(\mathcal{A}_{\lambda}^{-}(n, r)\right)$-tilt $\stackrel{\sim}{\rightarrow} \mathcal{O}_{\nu}\left(\mathcal{A}_{\lambda}(n, r)\right)$-inj, we see that $\operatorname{Res}_{\tau}$ maps injectives to injectives.

Step 4. To see that $\operatorname{Res}_{\tau}$ maps projectives to projectives, one can argue similarly by using the functor $=\mathfrak{W C C}_{\lambda^{-} \leftarrow \lambda}^{-1}=R \operatorname{Hom}_{\mathcal{A}_{\lambda^{-}}(n, r)}\left(\mathcal{A}_{\lambda, \lambda^{-}-\lambda}(n, r), \bullet\right)$ instead of $\mathcal{A}_{\lambda^{-}, \lambda-\lambda^{-}}^{0}(n, r) \otimes_{\mathcal{A}_{\lambda^{-}}(n, r)}^{L}$

6.3. Supports for asymptotic chambers. Recall that the simples of $\mathcal{O}_{\nu, \lambda}(n, r)$ are indexed by the $r$-multipartitions $\sigma=\left(\sigma^{(1)}, \ldots, \sigma^{(r)}\right)$ of $n$, moreover, $L_{\nu}(\sigma)=L_{\nu_{0}}(\underline{L}(\sigma))$, where $\underline{L}(\sigma)=L^{A}\left(\sigma^{(1)}\right) \otimes \ldots \otimes L^{A}\left(\sigma^{(r)}\right)$. We write $L^{A}\left(\sigma^{\prime}\right)$ for the irreducible module in the category $\mathcal{O}$ for the Rational Cherednik algebra $e H_{1, \lambda}\left(\left|\sigma^{\prime}\right|\right) e$, here $\sigma^{\prime}$ is a partition.

Here is our main result of this section, it describes the dimensions of the supports of the simples $L_{\nu}(\sigma)$ in terms of $\sigma$.

Theorem 6.10. Let $m$ denote the denominator of $\lambda$ (equal to $+\infty$ if $\lambda \notin \mathbb{Q}$ ). Assume $m>1$. Divide $\sigma^{(1)}$ by $m$ with remainder: $\sigma^{(1)}=m \sigma^{(1) q}+\sigma^{(1) r}$ (componentwise operations, 
" $q$ " and " $r$ " stand for the quotient and the remainder). Then $\operatorname{dim} \operatorname{Supp} L_{\nu}(\sigma)=r n-$ $\left|\sigma^{(1) q}\right|(r m-1)$.

Proof. Our proof is by induction on $n$. For $n<m$, the category $\mathcal{O}$ is semisimple, and all simples have support of dimension $r n$, while for $n=m$, the result follows from Theorem 4.3. So we will assume that the claim of the theorem is proved for all dimensions less than $n$.

Let us write $n_{i}$ for $\left|\sigma^{(i)}\right|$. By [L6, Theorem 1.2], all irreducible components of Supp $L_{\nu}(\sigma)$ have the same dimension. So if $\operatorname{Res}_{\tau}\left(L_{\nu}(\sigma)\right) \neq 0$, then $\operatorname{dim} \operatorname{Supp} L_{\nu}(\sigma)=\operatorname{dim} \operatorname{Res}_{\tau}\left(L_{\nu}(\sigma)\right)$. Recall, Corollary 6.8, that

$$
\operatorname{Res}_{\tau}\left(L_{\nu}(\sigma)\right)=L_{\nu}\left(\underline{\operatorname{Res}}_{\tau}\left(L^{A}\left(\sigma^{(1)}\right) \otimes L^{A}\left(\sigma^{(2)}\right) \otimes \ldots \otimes L^{A}\left(\sigma^{(r)}\right)\right)\right) .
$$

Let us first take $\tau=(n-1,1)$. Let us compute $\underline{\operatorname{Res}}_{\tau}\left(L^{A}\left(\sigma^{(1)}\right) \otimes L^{A}\left(\sigma^{(2)}\right) \otimes \ldots \otimes L^{A}\left(\sigma^{(r)}\right)\right)$. According to (6.3) it equals

$$
\bigoplus_{i=1}^{r} L^{(A)}\left(\sigma^{(1)}\right) \otimes \ldots \otimes E L^{(A)}\left(\sigma^{(i)}\right) \otimes \ldots \otimes L^{(A)}\left(\sigma^{(r)}\right),
$$

where we write $E$ for the Bezrukavnikov-Etingof functor restricting from $S_{n_{i}}$ to $S_{n_{i}-1}$. It is (up to a category equivalence) the sum of categorification functors $E_{i}$ for a categorical action of $\hat{\mathfrak{s l}}_{m}$ on $\bigoplus_{k=0}^{+\infty} \mathcal{O}\left(H_{\lambda+i-1}(k)\right)$. Let us divide $\sigma^{(i)}$ by $m$ with remainder: $\sigma^{(i)}=$ $m \sigma^{(i) q}+\sigma^{(i) r}$. We have $E L^{A}\left(\sigma^{(i)}\right)=0$ if and only if $\sigma^{(i) r}=0$, Wi]. Moreover, if $\sigma^{(i) r} \neq 0$, then $E L\left(\sigma^{(i)}\right)$ surjects onto $L\left(\underline{\sigma}^{(i)}\right)$, where $\underline{\sigma}^{(i)}$ is obtained from $\sigma^{(i)}$ by removing a box from $\sigma^{(i) r}$, this follows from results of [L3].

So assume that $\sigma^{(i) r} \neq \varnothing$ for some $i$. Let $\underline{\sigma}$ be the $r$-partition obtained from $\sigma$ by replacing $\sigma^{(i)}$ with $\underline{\sigma}^{(i)}$. Then $\operatorname{Res}_{\tau}(L(\sigma)) \rightarrow L(\underline{\sigma}) \otimes L$, where $L$ is some simple object in $\mathcal{O}_{\nu}\left(\mathcal{A}_{\lambda}(1, r)\right)$. By our inductive assumption, $\operatorname{dim} \operatorname{Supp} L(\underline{\sigma}) \otimes L=r n-(r m-1)\left|\sigma^{(1) q}\right|$. It follows that $\operatorname{dim} \operatorname{Supp} L(\sigma)=\operatorname{dim} \operatorname{Res}_{\tau}(L(\sigma)) \geqslant r n-(r m-1)\left|\sigma^{(1) q}\right|$. On the other hand, if $L^{A}\left(\hat{\sigma}^{(i)}\right)$ is a simple occurring in $E L^{A}\left(\sigma^{(i)}\right)$, then $\left|\hat{\sigma}^{(i) q}\right| \geqslant\left|\sigma^{(i) q}\right|$. From here we deduce that $\operatorname{dim} \operatorname{Supp} \operatorname{Res}_{\tau}(L(\sigma)) \leqslant r n-(r m-1)\left|\sigma^{(1) q}\right|$.

So we only need to consider the case when $\operatorname{Res}_{\tau}(L(\sigma))=0$. By the previous paragraph, this means that all $\sigma^{(i)}$ are divisible by $m$. Take $\tau^{\prime}=\left(\mathrm{m}^{n / m}\right)$. Then, since the support of $L^{A}\left(\sigma^{(i)}\right)$ corresponds to the partition $m^{n_{i} / m}$ of $n_{i}$, we can use (6.3) to get

$$
\underline{\operatorname{Res}}_{\tau^{\prime}}\left(L^{A}\left(\sigma^{(1)}\right) \otimes \ldots \otimes L^{A}\left(\sigma^{(r)}\right)\right)=\operatorname{Res} L^{A}\left(\sigma^{(1)}\right) \otimes \ldots \otimes \operatorname{Res} L^{A}\left(\sigma^{(r)}\right),
$$

where we write Res for the Bezrukavnikov-Etingof restriction functor from $S_{m n_{i}}$ to $S_{m}^{n_{i} / m}$. According to [Wi], we have $\operatorname{Res} L^{A}\left(\sigma^{(i)}\right)=L^{A}(m)^{\bigotimes n_{i} / m} \otimes V_{\sigma^{(i) q}}$, where $V_{\sigma^{(i) q}}$ is a nonzero multiplicity space (that is the irreducible $S_{\left|\sigma^{(i) q}\right|}$-module corresponding to $\sigma^{(i) q}$ ).

It follows that $\operatorname{Res}_{\tau^{\prime}} L(\sigma)$ is the sum of several copies of $L_{1}^{\bigotimes n_{1} / m} \otimes L_{2}^{\otimes n_{2} / m} \otimes \ldots \otimes L_{r}^{\otimes n_{r} / m}$. Here we write $L_{i}$ for the irreducible in $\mathcal{O}_{\nu}\left(\mathcal{A}_{\lambda}(m, r)\right)$ corresponding to the $r$-partition with $i$ th part $(m)$. We have $\operatorname{dim} \operatorname{Supp} L_{1}=1$ and $\operatorname{dim} \operatorname{Supp} L_{i}=r m$. Since $\operatorname{dim} \operatorname{Supp} L(\sigma)=$ $\operatorname{dim} \operatorname{Supp} \operatorname{Res}_{\tau^{\prime}} L(\sigma)$, this implies the claim of the theorem in this case and finishes the proof.

Proof of Theorem 1.5. In the proof we can assume that $\lambda$ is sufficiently big. By [L6, Theorem 1.2], we have $\operatorname{dim} \operatorname{Supp} L_{\nu}(\sigma)=\frac{1}{2} \operatorname{dim} \mathrm{V}\left(\mathcal{A}_{\lambda}(n, r) / \operatorname{Ann} L_{\nu}(\sigma)\right)$. Theorem 1.3 implies that the two-sided ideals in $\mathcal{A}_{\lambda}(n, r)$ are determined by the dimensions of their associated varieties. This finishes the proof of this theorem. 
6.4. Cross-walling bijections. We have computed the supports in the case when $\nu$ is dominant. For other chambers of one-parameter subgroups, supports can be computed using the cross-walling bijections defined in a more general situation below. We plan to compute these bijections combinatorially in a subsequent paper.

Let $X=X^{\theta}$ be a conical symplectic resolution equipped with a Hamiltonian action of a torus $T$ with finitely many fixed points. Let $\mathcal{A}_{\lambda}^{\theta}$ be a quantization of $X$ and $\mathcal{A}_{\lambda}:=\Gamma\left(\mathcal{A}_{\lambda}^{\theta}\right)$. We suppose that $\lambda$ is sufficiently ample so that Propositions 2.2 and 2.5 hold.

Let $\nu, \nu^{\prime}: \mathbb{C}^{\times} \rightarrow T$ be two generic one-parameter subgroups lying in chambers opposite with respect to a face. We are going to define a bijection $\mathfrak{c w}_{\nu^{\prime} \leftarrow \nu}: X^{T} \rightarrow X^{T}$. Let $\nu_{0}$ be a generic one-parameter subgroup in the common face of the chambers of $\nu, \nu^{\prime}$ such that these chambers are opposite with respect to the face. Consider the cross-walling functor $\underline{\mathfrak{C W}}_{\nu^{\prime} \leftarrow \nu}: D^{b}\left(\mathcal{O}_{\nu}\left(\mathrm{C}_{\nu_{0}}\left(\mathcal{A}_{\lambda}^{\theta}\right)\right)\right) \rightarrow D^{b}\left(\mathcal{O}_{\nu^{\prime}}\left(\mathrm{C}_{\nu_{0}}\left(\mathcal{A}_{\lambda}^{\theta}\right)\right)\right)$. For $\left.X^{\nu_{0}} \mathbb{C}^{\times}\right)$, the one-parameter subgroups $\nu, \nu^{\prime}$ lie in opposite chambers. So $\mathfrak{C W}_{\nu^{\prime} \leftarrow \nu}^{-1}$ is the direct sum (over the irreducible components of $X^{\nu_{0}\left(\mathbb{C}^{\times}\right)}$) of Ringel duality functors with various homological shifts. Each Ringel duality functor is a perverse equivalence, Lemma 2.8, and hence gives rise to a bijection between the set of simples, see Section 2.4. We take the disjoint union of these bijections for $\mathfrak{c w}_{\nu^{\prime} \leftarrow \nu}$.

The main result of this section is the following.

Proposition 6.11. We have $\operatorname{Ann}\left(L_{\nu}(p)\right)=\operatorname{Ann}\left(L_{\nu^{\prime}}\left(\mathfrak{c w}_{\nu^{\prime} \leftarrow \nu}(p)\right)\right)$ and $\operatorname{dim} \operatorname{Supp} L_{\nu}(p)=$ $\operatorname{dim} \operatorname{Supp} L_{\nu^{\prime}}\left(\mathfrak{c w}_{\nu^{\prime} \leftarrow \nu}(p)\right)$.

Proof. Note that the former equality implies the latter by Lemma 2.3.

Let us prove the equality of the annihilators. Recall that $\mathfrak{C} \mathfrak{W}_{\nu^{\prime} \leftarrow \nu} \circ \Delta_{\nu_{0}} \cong \Delta_{\nu_{0}} \circ \underline{\mathfrak{C W}}_{\nu^{\prime} \leftarrow \nu}$, Proposition 2.6. By the construction, $\underline{L}_{\nu^{\prime}}\left(\mathfrak{c w}_{\nu^{\prime} \leftarrow \nu}(p)\right)$ is a constituent in $H_{\bullet}\left(\underline{\mathfrak{C W}}_{\nu^{\prime} \leftarrow \nu} \underline{L}_{\nu}(p)\right)$. It follows that $L_{\nu^{\prime}}\left(\mathfrak{c w}_{\nu^{\prime} \leftarrow \nu}(p)\right)$ is a constituent in $H_{\bullet}\left(\mathfrak{C W}_{\nu^{\prime} \leftarrow \nu} L_{\nu}(p)\right)$. Set $\mathcal{I}:=\bigcap_{n} \operatorname{Ann} L_{\nu}(p)^{n}$ so that $\mathcal{I}^{2}=\mathcal{I}$. Since the regular $\mathcal{A}_{\lambda}$-bimodule has finite length, see Lemma 3.8, we see that $\mathcal{I}$ coincides with some power of Ann $L_{\nu}(p)$. It is enough to show that $H_{\bullet}\left(\mathfrak{C W}_{\nu^{\prime} \leftarrow \nu} L_{\nu}(p)\right)$ is annihilated by $\mathcal{I}$.

For any $\mathrm{HC} \mathcal{A}_{\lambda^{-}}$-bimodule $\mathcal{B}$ we have $\mathcal{B} \otimes_{\mathcal{A}_{\lambda}}^{L} \mathfrak{C W}_{\nu^{\prime} \leftarrow \nu}(\bullet) \cong \mathfrak{C W}_{\nu^{\prime} \leftarrow \nu}\left(\mathcal{B} \otimes_{\mathcal{A}_{\lambda}}^{L} \bullet\right)$. This is because the functors $\mathcal{B} \otimes_{\mathcal{A}_{\lambda}}^{L} \bullet, R \operatorname{Hom}_{\mathcal{A}_{\lambda}}(\mathcal{B}, \bullet)$ preserve the categories $D^{b}\left(\mathcal{O}_{\nu^{\prime \prime}}\right)$ for all generic $\nu^{\prime \prime}$.

We will apply the previous paragraph to $\mathcal{B}=\mathcal{I}$. We have

$$
\mathcal{I}^{\otimes^{L} k} \otimes^{L} \mathfrak{C W}_{\nu^{\prime} \leftarrow \nu}\left(L_{\nu}(p)\right) \cong \mathfrak{C W}_{\nu^{\prime} \leftarrow \nu}\left(\mathcal{I}^{\otimes^{L}, k} \otimes^{L} L_{\nu}(p)\right)
$$

where all derived tensor products are taken over $\mathcal{A}_{\lambda}$ and we write $\mathcal{I}^{{ }^{L}} k$ for the $k$-th derived tensor power of $\mathcal{I}$. Note that thanks to $\mathcal{I}^{2}=\mathcal{I}$ we get $\mathcal{I} \otimes_{\mathcal{A}_{\lambda}} \mathcal{A}_{\lambda} / \mathcal{I}=0$. It follows that $\mathcal{I} \otimes_{\mathcal{A}_{\lambda}} M=0$ for all $\mathcal{A}_{\lambda}$-modules $M$ annihilated by $\mathcal{I}$. Moreover, thanks to the exact sequence $0 \rightarrow \mathcal{I} \rightarrow \mathcal{A}_{\lambda} \rightarrow \mathcal{A}_{\lambda} / \mathcal{I} \rightarrow 0$, all homology of $\mathcal{I} \otimes_{\mathcal{A}_{\lambda}}^{L} M$ are annihilated by $\mathcal{I}$ provided $M$ is annihilated by $\mathcal{I}$. We deduce that $H_{i}\left(\mathcal{I}^{\otimes^{L}, k} \otimes^{L} L_{\nu}(p)\right)=0$ for $i<k$. We deduce that the homology of the right hand side of (6.5) vanishes in degrees $\leqslant k-1$. On the other hand, let $k$ be the minimal number such that $H_{\ell}\left(\mathfrak{C W}_{\nu^{\prime} \leftarrow \nu}\left(L_{\nu}(p)\right)\right)$ is not annihilated by any power of $\mathcal{I}$. Using the spectral sequence for the composition of derived functors, we see that $H_{\ell}$ of the left hand side of (6.5) is nonzero for any $k$. This gives a contradiction that completes the proof. 


\section{REFERENCES}

[BEG] Yu. Berest, P. Etingof, V. Ginzburg, Finite-dimensional representations of rational Cherednik algebras. Int. Math. Res. Not. 2003, no. 19, 1053-1088.

[BE] R. Bezrukavnikov, P. Etingof, Parabolic induction and restriction functors for rational Cherednik algebras. Selecta Math., 14(2009), 397-425.

[BeKa] R. Bezrukavnikov, D. Kaledin. Fedosov quantization in the algebraic context. Moscow Math. J. 4 (2004), 559-592.

[BL] R. Bezrukavnikov, I. Losev, Etingof conjecture for quantized quiver varieties. arXiv:1309.1716.

[BoKr] W. Borho, H. Kraft. Über die Gelfand-Kirillov-Dimension. Math. Ann. 220(1976), 1-24.

[BLPW] T. Braden, A. Licata, N. Proudfoot, B. Webster, Quantizations of conical symplectic resolutions II: category $O$ and symplectic duality. arXiv:1407.0964. To appear in Astérisque.

[BPW] T. Braden, N. Proudfoot, B. Webster, Quantizations of conical symplectic resolutions I: local and global structure. arXiv:1208.3863. To appear in Astérisque.

[CB] W. Crawley-Boevey, Geometry of the moment map for representations of quivers, Comp. Math. 126 (2001), 257-293.

[EG] P. Etingof and V. Ginzburg. Symplectic reflection algebras, Calogero-Moser space, and deformed Harish-Chandra homomorphism, Invent. Math. 147 (2002), 243-348.

[GG] W.L. Gan, V. Ginzburg, Almost commuting variety, D-modules and Cherednik algebras. IMRP, 2006, doi: 10.1155/IMRP/2006/26439.

[GS1] I. Gordon, T. Stafford, Rational Cherednik algebras and Hilbert schemes, Adv. Math. 198 (2005), no. $1,222-274$.

[GS2] I. Gordon, T. Stafford, Rational Cherednik algebras and Hilbert schemes. II. Representations and sheaves, Duke Math. J. 132 (2006), no. 1, 73135.

[KR] M. Kashiwara and R. Rouquier, Microlocalization of rational Cherednik algebras, Duke Math. J. 144 (2008) 525-573.

[Ko] D. Korb. Order on the Fixed Points of the Gieseker Variety With Respect to the Torus Action. arXiv:1312.3025

[L1] I. Losev, Completions of symplectic reflection algebras. Selecta Math., 18(2012), N1, $179-251$. arXiv:1001.0239.

[L2] I. Losev. Isomorphisms of quantizations via quantization of resolutions. Adv. Math. 231(2012), 12161270 .

[L3] I. Losev, Highest weight $\mathfrak{s l}_{2}$-categorifications I: crystals. Math. Z. 274(2013), 1231-1247.

[L4] I. Losev. Abelian localization for cyclotomic Cherednik algebras. Int Math Res Notices (2015) vol. 2015, 8860-8873.

[L5] I. Losev. On categories $\mathcal{O}$ for quantized symplectic resolutions. arXiv:1502.00595.

[L6] I. Losev. Bernstein inequality and holonomic modules (with a joint appendix by I. Losev and P. Etingof). arXiv:1501.01260.

[L7] I. Losev. Wall-crossing functors for quantized symplectic resolutions: perversity and partial Ringel dualities. arXiv:1604.06678

[LW] I. Losev, B. Webster, On uniqueness of tensor products of irreducible categorifications. Selecta Math. 21(2015), N2, 345-377.

[MN1] K. McGerty, T. Nevins, Derived equivalence for quantum symplectic resolutions, Selecta Math. (N.S.) 20 (2014), no. 2, 675-717.

[MN2] K. McGerty, T. Nevins, Compatability of t-structures for quantum symplectic resolutions. Duke Math J. 165(2016), 2529-2585.

[Nak] H. Nakajima. Quiver varieties and tensor products. Invent. Math. 146 (2001), no. 2, $399-449$.

[NY] H. Nakajima, K. Yoshioka. Lectures on Instanton Counting. Algebraic structures and moduli spaces, 31101, CRM Proc. Lecture Notes, 38, Amer. Math. Soc., Providence, RI, 2004.

[Nam] Y. Namikawa. Poisson deformations and Mori dream spaces, arXiv:1305.1698.

[R] R. Rouquier, q-Schur algebras for complex reflection groups. Mosc. Math. J. 8 (2008), 119-158.

[S] P. Shan. Crystals of Fock spaces and cyclotomic rational double affine Hecke algebras. Ann. Sci. Ecole Norm. Sup. 44 (2011), 147-182.

[We] B. Webster. On generalized category $\mathcal{O}$ for a quiver variety. arXiv:1409.4461.

[Wi] S. Wilcox, Supports of representations of the rational Cherednik algebra of type A. arXiv:1012.2585. 
Department of Mathematics, Northeastern University, Boston MA 02115 USA

E-mail address: i.loseu@neu.edu 